\title{
Stellar and wind parameters of Galactic O-stars
}

\section{The influence of line-blocking/blanketing ${ }^{\star, \star \star}$}

\author{
T. Repolust ${ }^{1}$, J. Puls ${ }^{1}$, and A. Herrero ${ }^{2,3}$ \\ 1 Universitäts-Sternwarte München, Scheinerstr. 1, 81679 München, Germany \\ 2 Instituto de Astrofísica de Canarias, 38200 La Laguna, Tenerife, Spain \\ 3 Departamento de Astrofísica, Universidad de La Laguna, Avda. Astrofísico Francisco Sánchez, s/n, 38071 La Laguna, Spain
}

Received 20 May 2003 / Accepted 17 October 2003

\begin{abstract}
We have re-analyzed the Galactic O-star sample from Puls et al. (1996) by means of line-blanketed NLTE model atmospheres in order to investigate the influence of line-blocking/blanketing on the derived parameters. The analysis has been carried out by fitting the photospheric and wind lines from $\mathrm{H}$ and $\mathrm{He}$. In most cases we obtained a good fit, but we have also found certain inconsistencies which are probably related to a still inadequate treatment of the wind structure. These inconsistencies comprise the line cores of $\mathrm{H}_{\gamma}$ and $\mathrm{H}_{\beta}$ in supergiants (the synthetic profiles are too weak when the mass-loss rate is determined by matching $\mathrm{H}_{\alpha}$ ) and the "generalized dilution effect" (cf. Voels et al. 1989) which is still present in He I 4471 of cooler supergiants and giants.

Compared to pure $\mathrm{H} / \mathrm{He}$ plane-parallel models we found a decrease in effective temperatures which is largest at earliest spectral types and for supergiants (with a maximum shift of roughly $8000 \mathrm{~K}$ ). This finding is explained by the fact that line-blanketed models of hot stars have photospheric He ionization fractions similar to those from unblanketed models at higher $T_{\text {eff }}$ and higher $\log g$. Consequently, any line-blanketed analysis based on the He ionization equilibrium results in lower $T_{\text {eff }}$-values along with a reduction of either $\log g$ or helium abundance (if the reduction of $\log g$ is prohibited by the Balmer line wings). Stellar radii and mass-loss rates, on the other hand, remain more or less unaffected by line-blanketing.

We have calculated "new" spectroscopic masses and compared them with previous results. Although the former mass discrepancy (Herrero et al. 1992) becomes significantly reduced, a systematic trend for masses below $50 M_{\odot}$ seems to remain: The spectroscopically derived values are smaller than the "evolutionary masses" by roughly $10 M_{\odot}$. Additionally, a significant fraction of our sample stars stays over-abundant in $\mathrm{He}$, although the actual values were found to be lower than previously determined.

Also the wind-momentum luminosity relation (WLR) changes because of lower luminosities and almost unmodified windmomentum rates. Compared to previous results, the separation of the WLR as a function of luminosity class is still present but now the WLR for giants/dwarfs is consistent with theoretical predictions.

We argue that the derived mass-loss rates of stars with $\mathrm{H}_{\alpha}$ in emission are affected by clumping in the lower wind region. If the predictions from different and independent theoretical simulations (Vink et al. 2000; Pauldrach et al. 2003; Puls et al. 2003a) that the WLR should be independent of luminosity class were correct, a typical clumping factor $\left\langle\rho^{2}\right\rangle /\langle\rho\rangle^{2} \approx 5$ should be derived by "unifying” the different WLRs.
\end{abstract}

Key words. stars: atmospheres - stars: distances - stars: early-type - stars: fundamental parameters - stars: mass loss stars: winds, outflows

\section{Introduction}

The understanding of massive stars and their evolution is not only fundamental to stellar astrophysics but it also provides

Send offprint requests to: T. Repolust, e-mail: repo@usm.uni-muenchen.de

* Based upon observations obtained at the INT and the European Southern Observatory, La Silla, Chile. The INT is operated on the island of La Palma by the ING in the Spanish Observatorio de El Roque de los Muchachos of the Instituto de Astrofísica de Canarias.

$\star \star$ Appendix A in only available in electronic form at http://www. edpsciences.org insights into a variety of other processes. Massive star winds (with parameters depending on evolutionary stage and environment) are crucial for the chemical and dynamical evolution of galaxies through their input of energy, momentum, and nuclear processed material into the interstellar medium (e.g., Leitherer \& Heckman 1995; Silich \& Tenorio-Tagle 2001; Oey 2003). In the distant Universe, massive stars dominate the integrated UV-light of very young galaxies (Steidel et al. 1996; Pettini et al. 2000); even earlier they are the suspected sources of the re-ionization of the Universe (Bromm et al. 2001).

Thus, by observing and analyzing massive stars we can (in principle) provide the numbers required to tackle these 
problems. Moreover, we can address a number of ensuing questions which are of great interest and might have significant consequences for our general understanding. Present efforts, for example, concentrate on the physics of rotation (with respect to both the interior structure by means of mixing processes and the exterior structure by modifying the wind morphology, e.g. Maeder \& Meynet 2000 and references therein), the influence of time-dependent processes (see below) and the so-called wind-momentum luminosity relation (WLR) which might become an independent tool to derive extragalactic distances on an intermediate scale (up to the Virgo and Fornax cluster).

This knowledge is mainly derived from the analysis of stellar spectra which in turn requires adequate atmospheric models. For this purpose sophisticated model atmosphere codes have been developed in the last decade, e.g., Hubeny \& Lanz (1995), Santolaya-Rey et al. (1997, "FASTWIND"), Hillier et al. (1998), Pauldrach et al. (2001) and Gräfener et al. (2002) which incorporate detailed atomic models and improved numerical techniques. These models offer us the opportunity to derive rather realistic stellar parameters and provide insight into the chemical composition of stars. Additionally, the latter four codes allow for an investigation of important wind properties such as mass-loss rates, wind terminal velocities and velocity structures.

Considering that the actual value of the mass-loss rate has a significant influence on massive star evolution ${ }^{1}$, the derived mass-loss rates need to be known to a level of precision better than a factor of two; otherwise, evolutionary calculations relying on these numbers could become completely erroneous.

Although such a precision is feasible (at least differentially, cf. Kudritzki \& Puls 2000 and references therein), the situation looks different on an absolute scale. Most important in this sense is the fact that stellar rotation (e.g., Maeder \& Meynet 2000a), the intrinsic instability of the line-driving mechanism (Owocki et al. 1988; Feldmeier 1995; Owocki \& Puls 1999) and their interaction (Owocki 1999) are able to produce nonspherical and inhomogeneous structures, observationally evident, e.g., from X-ray emission and line profile variability. Unless we completely understand these structures, we cannot be entirely sure about the "average" properties of stellar winds like mass-loss rates and ionizing fluxes. At least in the case of WR-stars, the presence of clumping has severe consequences for the interpretation of observed line profiles, particularly with respect to the derived mass-loss rates, e.g., Moffat \& Robert (1994), Schmutz (1997).

Not only do present mass-loss rates remain somewhat uncertain but also the basic stellar parameters of O-stars are subjected to a number of uncertainties. Using plane-parallel NLTE-models, Herrero et al. (1992) have presented discrepancies in masses and helium abundances of O-stars, derived from either evolutionary tracks or spectroscopy. It was suggested that these discrepancies could be reduced by accounting for rotational mixing in the evolutionary calculations (which partly turned out to be true, Meynet \& Maeder 2000) and/or by

\footnotetext{
${ }^{1}$ Note that, e.g., for O-stars the product of typical mass-loss rate times hydrogen burning life-time is a significant fraction of total mass.
}

including the effects of mass-loss and sphericity into the atmospheric models.

The latter deficiency was corrected when so-called unified model atmospheres became available (Gabler et al. 1989). On the basis of these models, Puls et al. (1996) introduced an approximate method to obtain mass-loss estimates for a large sample of Galactic and Magellanic Cloud O-stars. As a "by-product", this analysis provided the basis for the windmomentum luminosity relationship of hot stars (Kudritzki et al. 1995).

There is, however, one additionally important effect that could not be treated at that time: the influence of lineblocking/blanketing which should significantly affect the analysis, mainly with respect to the temperature scale as a function of spectral type. With the present generation of atmospheric model codes this task has now become feasible.

Recently Martins et al. (2002) have presented such a new temperature scale for massive $\mathrm{O} d$ warfs that is actually considerably lower than the one found by Vacca et al. (1996) (based on plane-parallel, pure $\mathrm{H} / \mathrm{He}$ model atmospheres) as a result of strong metal line blanketing. Crowther et al. (2002) have presented an analysis of four supergiants in the LMC and SMC with similar but stronger trends, and Herrero et al. (2002) have analyzed seven Cyg OB2 stars by means of the latest, lineblanketed version of FASTWIND.

In view of the important role of hot stars and their winds, a re-analysis of the O-star sample by Puls et al. (1996) is urgently required, particularly because this sample still comprises the basic data set for O-star mass-loss rates and corresponding WLRs.

In the present paper we will first concentrate on the Galactic sub-sample and on a detailed spectral analysis of the corresponding stars and then comment on peculiarities, problems and uncertainties, mainly on the basis of a differential comparison with previous results from unblanketed models. Preliminary results of our investigation have already been published in Puls et al. (2003a).

The remainder of this paper is organized as follows. In Sect. 2 we briefly outline the characteristics of the code used, followed by a description of our Galactic sample in Sect. 3 . Section 4 comprises general remarks on our procedure and summarizes the basic results of our analysis in Table 1. In Sect. 5, we comment in detail on our individual objects and in Sect. 6 we present an elaborated error analysis. In Sect. 7 we discuss the implications of our investigation: First, we consider the relation between effective temperature vs. spectral type and gravity vs. effective temperature in view of the new results. We then explain the differences in the results from blanketed and unblanketed models in fair detail and comment on the status quo of mass and helium discrepancies outlined above and present an updated view of the WLR for Galactic O-type stars. Finally, Sect. 8 comprises further conclusions and a summary of this work.

\section{The code}

In order to investigate the influence of line-blocking/blanketing on $T_{\text {eff }}$, and subsequently on the other stellar and wind 
parameters, we have begun to re-analyze the O-star sample compiled by Puls et al. (1996, in the following Paper I). As outlined above, in the present paper we will concentrate on the Galactic objects of this sample, where the analysis will be performed by means of NLTE-atmospheres/line formation, utilizing the latest version of FASTWIND (an acronym for Fast Analysis of STellar atmospheres with WINDs; Herrero et al. 2002; Santolaya-Rey et al. 1997). This code, allowing for the simultaneous computation of photospheric and wind lines, has the enormous advantage of being very fast (roughly $30 \mathrm{~min} /$ model on any $1 \mathrm{GHz}$ processor), enabling us to calculate the vast amount of models required. This computational efficiency is obtained by applying appropriate physical approximations to certain processes where high accuracy is not needed (regarding the objective of the analysis - optical lines!), in particular for the treatment of the metal-line background opacities.

The code comprises the concept of "unified model atmospheres" (i.e., the smooth transition from a pseudo-hydrostatic photosphere to a wind) along with an appropriate treatment of line-broadening which is a prerequisite for the analysis of O-stars of different luminosity classes covering a variety of wind densities.

The approximations underlying the treatment of metal line blocking/blanketing will be described in detail by Puls et al. (2003b, in prep.). In summary, the calculation of the required NLTE metal opacities (bound-bound, bound-free, and freefree) follows the principal philosophy presented by Abbott \& Lucy (1985), Schmutz (1991), Schaerer \& Schmutz (1994) and Puls et al. (2000) and important details have now been improved upon. Particularly, the equations of approximate ionization equilibrium have been re-formulated to account for the actual radiation field as a function of depth at all ionization edges, and a consistent iteration scheme regarding the coupling of the rate equations and the radiation field has been established to avoid the well-known convergence problems of a pure Lambda Iteration. For the calculation of the effects of line-blocking, we used suitable means for the line opacities, averaged over a frequency interval of the order of $v_{\infty}$, while flux conservation (line-blanketing!) is obtained by incorporating the concept of NLTE-Hopf parameters (cf. Santolaya-Rey et al. 1997) in a consistent way.

The code has carefully been tested by comparison with results from alternative, fully blanketed codes presently available. On the one hand, we have compared our blanketed fluxes with those from the model-grid ${ }^{2}$ provided by Pauldrach et al. (2001, WMbasic), and found very good agreement in almost all cases. Some of the complementary tests, on the other hand, have already been discussed by Herrero et al. (2002, see also Urbaneja et al. 2003). As an example for stars with negligible winds, the analysis of $10 \mathrm{Lac}(\mathrm{O} 9 \mathrm{~V})$ resulted in an excellent fit at temperatures lower than those obtained from unblanketed models, and the derived parameters completely agree with those obtained by Hubeny et al. (1998) using TLUSTY. With respect to CMFGEN, direct as well as indirect tests have

\footnotetext{
${ }^{2}$ Comprising six supergiants and six dwarfs between 30000 and $50000 \mathrm{~K}$, see also http://www.usm. uni-muenchen.de/people /adi/Models/Model.html
}

been performed. As an example of direct tests, Herrero et al. (2002) have compared the emergent fluxes resulting from FASTWIND and CMFGEN for a model of Cyg OB2 \#7 (O3 If*), and again found remarkable agreement between both codes for this hot supergiant with strong wind. Unpublished indirect tests (Najarro, priv. commun.) concern an alternative combined UV/optical analysis, performed for a part of the Cyg OB2 objects from Herrero et al. (2002) by means of CMFGEN. For two objects (Cyg OB2 \#4 (O7 III ((f))) and \#10 (O9.5 I) excellent agreement in all derived parameters was obtained, whereas for two other objects (Cyg OB2 \#11 (O5 If*) and \#8A (O5.5 I(f)) somewhat cooler temperatures (and accordingly also lower mass-loss rates) have been derived. The origin of this inconsistency (the only one arising so far) is still unclear and will be analyzed in a forthcoming investigation. Note, however, that an independent CMFGEN analysis of $\zeta$ Pup (O4 I(f)) performed by Crowther et al. (2002) resulted in very similar parameters as found in the present work (cf. Sect. 7.1).

\section{The O-star sample}

For our re-analysis we have used the spectra described by Herrero et al. (1992) and Puls et al. (1996). The $\mathrm{H}_{\alpha}$ observations of the core sample of Galactic O-stars were taken from Herrero et al. (1992) and Herrero (1993) and were carried out with the $2.5 \mathrm{~m}$ Isaac Newton telescope at the Observatory of El Roque de los Muchachos in La Palma in July and October 1989 and in August 1992. The blue observations needed to derive photospheric parameters were obtained during the same runs with an additional one in September 1991. Note however that the blue and red spectra were not taken simultaneously. For a specific observational run, all red spectra were obtained during one night, whereas the blue spectra were collected during the remaining nights.

The Intermediate Dispersion Spectrograph (IDS) was used with the $1800 \mathrm{~V}$ grating along with the $235 \mathrm{~mm}$ camera yielding a spectral resolution of $0.8 \AA$ FWHM along with a measured $\mathrm{S} / \mathrm{N}$ ratio of $\approx 300$ and a spectral resolution of $0.6 \AA$ FWHM along with a S/N ratio ranging from 150 to 200 for the red and the blue observations, respectively. The reduction of the data was made following standard procedures (using various packages such as IRAF, FIGARO (Shortridge 1987), Midas etc.) comprising bias subtraction, flat field division, spectrum extraction, wavelength calibration and continuum rectification.

The above data is supplemented by additional O3 stars located in the Carina nebula and some further well-observed stars such as $\zeta$ Pup and $\alpha$ Cam. The data for the Carina stars (HD 93129A, HD 93128, HD 93250, and HD 303308) were obtained in December 1992 using the ESO New Technology Telescope (NTT) and the EMMI spectrograph covering the wavelength ranges 3920-4380, 4300-4750 and 6300-6800 ̊ at a resolution of $0.9,0.95$ and $1.1 \AA$, respectively. The measured $\mathrm{S} / \mathrm{N}$ ratio was found to be of the order of $\approx 200$.

Furthermore, additional red spectrograms of HD 207198, and HD 209975 were obtained with a similar instrumental setup as described by Herrero et al. (1992) and Herrero (1993). 
The blue spectra of $\alpha$ Cam were taken from Lennon et al. (1992), and the red ones from Lennon et al. (1993).

The data for $\zeta$ Pup (blue and red), finally, was taken from Bohannan et al. (1990), where further information of the observational material and data reduction procedures may be found.

For all spectra we used the rectifications provided by the corresponding observers. Note, however, that particularly the region around the "new" He I/He II lines in the red band (see below) suffers from some problems in rectification, since this region has not been considered in detail before. Nevertheless, we have refrained from any "re-rectification" and have commented on the problem when present.

In total the sample consists of 24 Galactic O-stars as listed in Table 1 covering luminosity class I, III, and V objects.

\section{Analysis - General remarks}

Before presenting the detailed results of our analysis, we would like to remark on certain aspects concerning our procedure.

Micro-turbulence. As is well known, the inclusion of an adopted micro-turbulent velocity into the profile-functions can diminish certain discrepancies between the He I singlet and triplet lines in the B- and late O-star regime (cf. Smith \& Howarth 1998 and the discussion below), whereas for hotter O-stars micro-turbulence has (almost) no effect on the analysis (Villamariz \& Herrero 2000). Following these results, for almost all stars later than O6 (regardless of their luminosity class) we adopted a $v_{\text {turb }}$ of $10 \mathrm{~km} \mathrm{~s}^{-1}$ as a reasonable compromise. At spectral type O6, our analysis of HD 210839 (lc I) indicated that such a micro-turbulence is still needed, whereas for HD $217086(\mathrm{O} 7 \mathrm{~V})$, the inclusion of $v_{\text {turb }}$ did not change our results. Since both stars turned out to lie at $T_{\text {eff }}=36000 \mathrm{~K}$, we conclude this temperature to be an upper limit where microturbulence plays a role and is actually needed. For all stars hotter than O6, we adopted $v_{\text {turb }}=0$, in agreement with the results from Villamariz \& Herrero (2000).

Distances/Radii. It has recently been proposed that distances to open clusters derived from HIPPARCos observations might be systematically smaller than photometric ones (de Zeeuw et al. 1999). If this was confirmed, we would require a new calibration of absolute magnitudes in the upper part of the HR diagram. However, since present data is still scarce, we have systematically adopted photometric distances for stars belonging to $\mathrm{OB}$ associations (collected from different sources in the literature) to avoid an additional bias in our data.

Nevertheless, we still have to consider the runaway or field stars in the sample. Four of them have measured HIPPARCos parallaxes (ESA 1997) with not too large errors: HD 66811 ( $\zeta$ Pup), HD 210839 ( $\lambda$ Cep), HD 24912 ( $\xi$ Per) and HD 149757 ( $\zeta$ Oph). In these cases, however, we have to consider the uncertainty in the derived absolute magnitudes introduced by the Lutz-Kelker effect (Lutz \& Kelker 1973). Only $\zeta$ Oph has a relative error that allows a standard correction of the Lutz-Kelker effect and we adopt the value derived from the measured parallax and the correction provided by Koen (1992). We have reduced the $90 \%$ confidence limits provided by Koen to the usual standard deviation for our errors.

The relative error in the parallax of $\zeta$ Pup is slightly beyond the limit for which the standard Lutz-Kelker correction can be applied. We have estimated the correction using Fig. 2 in Oudmajier et al. (1998) and have found that the resulting value agrees well with existing calibrations of absolute magnitude versus spectral classification (e.g., Massey 1998 or Walborn 1972). Therefore, we have adopted this resulting value and corresponding uncertainty (i.e., $\pm 0.43 \mathrm{mag}$ which is larger than the uncertainty adopted for most of the stars).

We have performed the same exercise for $\lambda$ Cep, but the resulting value did not comply with current calibrations. We preferred the absolute magnitude from spectroscopic parallaxes given in the literature, in particular the value provided by Garmany \& Stencel (1992) since its agreement with existing calibrations is better. Additionally, we adopted a larger uncertainty in absolute magnitude, $\pm 0.5 \mathrm{mag}$.

$\xi$ Per has the largest relative error in the measured parallax and the absolute magnitudes found in the literature do not match its spectral classification (Humphreys 1978; de Zeeuw et al. 1999; Hoogerwerf et al. 2001). Therefore, we adopt a value from the calibrations by Massey (1998) and Walborn (1972).

For HD 30614 ( $\alpha$ Cam), finally, we have used the absolute magnitude taken from the calibration by Walborn $(1972)^{3}$.

Compared to the "old" values from Paper I, the "new" absolute magnitudes remain almost unchanged (typical modifications are of the order of \pm 0.1 . .0.15 mag) except for the following cases: $\xi$ Per, $\alpha$ Cam and HD 209975 have become brighter by $0.4,0.9$ and $0.7 \mathrm{mag}$, respectively, whereas HD 217086 has become fainter by $0.4 \mathrm{mag}$.

From these $M_{\mathrm{v}}$-values, stellar radii have been calculated following the procedure outlined by Kudritzki (1980) and Herrero et al. (1992):

$5 \log R_{\star}=29.58+\left(V_{\text {theo }}-M_{\mathrm{V}}\right)$,

$V_{\text {theo }}=-2.5 \log \int_{\text {filter }} 4 H_{\lambda} S_{\lambda} \mathrm{d} \lambda$,

where $H_{\lambda}$ is the theoretical Eddington flux from the calculated models (in units of [erg s $\mathrm{cm}^{-2} \AA^{-1}$ ]) and $S_{\lambda}$ is the spectral response of the photometric system.

The input radii used as starting values for our atmospheric models were taken from Paper I and have been calculated from the "old" $M_{\mathrm{v}}$-values provided by Herrero et al. (1992) and Paper I. Since the inclusion of line blocking/blanketing changes the theoretical fluxes (cf. Sect. 7.2) and since we have adopted somewhat different values for $M_{\mathrm{v}}$ (see above), the radii change accordingly which has been accounted for in the calculation of the final models. Even for the largest modifications of $M_{\mathrm{v}}$, the changes in radius remain below $25 \%$, except for $\alpha$ Cam, with an increase in radius by $50 \%$, cf. Table 1.

Note that in Table 1 all radius-dependent quantities such as luminosity, mass and mass-loss rate refer to the stellar radii calculated from the $M_{\mathrm{v}}$-values as described above (" $R_{*}$ "), since we

\footnotetext{
3 For a more thorough discussion concerning the problem of distances and magnitudes, we refer the reader to Markova et al. (2003).
} 
Table 1. Galactic O-star sample: Stellar and wind parameters adopted $\left(M_{\mathrm{v}}\right)$ and derived using FASTWIND. $T_{\mathrm{eff}}$ in $\mathrm{kK}, R_{*}$ in $R_{\odot}, V_{\mathrm{r}} \sin i$ and $v_{\infty}$ in $\mathrm{km} \mathrm{s}^{-1}, M_{*}$ in $M_{\odot}, L$ in $L_{\odot}, \dot{M}$ in $10^{-6} M_{\odot} / \mathrm{yr}$ (terminal velocities $v_{\infty}$ from Paper I). HD 93129A and HD 303308 have recently been detected as binary systems but they are treated here as single stars (see text). Bold face numbers denote $\beta$-values which could be derived with high precision from emission profiles. Stars with absorption profiles were fitted with an assumed value of $\beta=0.80$. $R_{\text {old }}$ is the stellar radius resulting from our new models which was calculated by means of the "old" $M_{\mathrm{v}}$ values from Paper I (not tabulated here).

\begin{tabular}{|c|c|c|c|c|c|c|c|c|c|c|c|c|c|c|}
\hline Star & Sp.Type & $M_{\mathrm{v}}$ & $T_{\text {eff }}$ & $\log g$ & $\log g_{\text {true }}^{2)}$ & $R_{*}$ & $Y_{\mathrm{He}}$ & $V_{\mathrm{r}} \sin i$ & $v_{\infty}$ & $\log L$ & $M_{*}$ & $\dot{M}$ & $\beta$ & $R_{\text {old }}$ \\
\hline HD 93129A ${ }^{1)}$ & O2 If* & -6.74 & 42.5 & 3.70 & 3.71 & $(22.5)$ & 0.10 & 130 & 3200 & $(6.17)$ & $(94.8)$ & $(26.30)$ & 0.80 & $(21.4)$ \\
\hline HD 93128 & O3 V ((f)) & -5.24 & 46.5 & 4.00 & 4.01 & 10.4 & 0.10 & 100 & 3100 & 5.66 & 39.8 & 2.64 & $0.85^{3)}$ & 10.2 \\
\hline HD 93250 & O3 V ((f)) & -6.14 & 46.0 & 3.95 & 3.96 & 15.9 & 0.10 & 130 & 3250 & 6.01 & 83.3 & 3.45 & $0.90^{3)}$ & 17.6 \\
\hline HD 66811 & O4 I(f) & -6.32 & 39.0 & 3.55 & 3.59 & 19.4 & 0.20 & 220 & 2250 & 5.90 & 53.9 & 8.80 & 0.90 & 16.8 \\
\hline HD $303308^{1)}$ & $\mathrm{O} 4 \mathrm{~V}\left(\left(\mathrm{f}^{+}\right)\right)$ & -5.29 & 41.0 & 3.90 & 3.91 & (11.5) & 0.075 & 120 & 3100 & $(5.53)$ & $(39.0)$ & (1.63) & 0.80 & (12.6) \\
\hline HD 14947 & $\mathrm{O} 5 \mathrm{If}^{+}$ & -5.94 & 37.5 & 3.45 & 3.48 & 16.8 & 0.20 & 140 & 2350 & 5.70 & 30.7 & 8.52 & 0.95 & 18.1 \\
\hline HD 15558 & O5 III(f) & -6.27 & 41.0 & 3.80 & 3.81 & 18.2 & 0.10 & 150 & 2800 & 5.93 & 78.7 & 5.58 & 0.80 & 19.4 \\
\hline HD 193682 & O5 III(f) & -5.55 & 40.0 & 3.60 & 3.65 & 13.1 & 0.20 & 200 & 2800 & 5.60 & 27.9 & 1.73 & 0.80 & 12.3 \\
\hline HD 1 & O5 V & -5.50 & 40.5 & 3.70 & 3.71 & 12.8 & 0.08 & 90 & 3200 & 5.60 & 30.4 & .28 & 0.80 & 12.8 \\
\hline HD 21 & O6 I & -6.40 & 36.0 & 3.55 & 358 & 21.1 & 0.10 & 200 & 2250 & 5.83 & 62.2 & 5.85 & 1.00 & 20.2 \\
\hline HD 190864 & O6.5 III(f) & -5.29 & 37.0 & 3.55 & 3.57 & 12.3 & 0.15 & 105 & 2500 & 5.41 & 20.3 & 1.39 & 0.80 & 14.2 \\
\hline HD 192639 & $\mathrm{O} 7 \mathrm{Ib}(\mathrm{f})$ & -6.10 & 35.0 & 3.45 & 3.47 & 18.7 & 0.20 & 125 & 2150 & 5.68 & 37.5 & 6.32 & 0.90 & 19.6 \\
\hline HD 193514 & $\mathrm{O} 7 \mathrm{Ib}(\mathrm{f})$ & -6.15 & 34.5 & 3.30 & 3.32 & 19.3 & 0.10 & 105 & 2200 & 5.68 & 28.2 & 3.48 & 0.80 & 19.7 \\
\hline HD 24912 & O7.5 III(n)((f)) & -5.50 & 35.0 & 3.50 & 3.56 & 14.0 & 0.15 & 220 & 2450 & 5.42 & 26.1 & 1.08 & 0.80 & 11.6 \\
\hline HD 203064 & O7.5 III:n ((f)) & -5.74 & 34.5 & 3.50 & 3.60 & 15.7 & 0.10 & 300 & 2550 & 5.50 & 35.9 & 1.41 & 0.80 & 14.1 \\
\hline HD 217086 & O7 V n & -4.50 & 36.0 & 3.50 & 3.72 & 8.6 & 0.15 & 350 & 2550 & 5.05 & 14.2 & $\leq 0.23$ & 0.80 & 10.4 \\
\hline HD 13268 & ON8 V & -4.77 & 33.0 & 3.25 & 3.48 & 10.3 & 0.25 & 300 & 2150 & 5.05 & 11.7 & $\leq 0.26$ & 0.80 & 11.4 \\
\hline HD 210809 & O9 Iab & -6.20 & 31.5 & 3.10 & 3.12 & 21.2 & 0.14 & 100 & 2100 & 5.60 & 21.7 & 5.30 & 0.90 & 21.2 \\
\hline HD 207198 & $\mathrm{O} 9 \mathrm{Ib}$ & -5.80 & 33.0 & 3.45 & 3.46 & 16.6 & 0.12 & 80 & 2150 & 5.47 & 29.0 & 1.79 & 0.80 & 14.5 \\
\hline HD 30614 & O9.5 Ia & -7.00 & 29.0 & 2.97 & 2.99 & 32.5 & 0.10 & 100 & 1550 & 5.83 & 37.6 & 6.04 & 1.15 & 21.5 \\
\hline HD 209975 & O9.5 Ib & -6.41 & 32.0 & 3.20 & 3.22 & 22.9 & 0.10 & 100 & 2050 & 5.69 & 31.4 & 2.15 & 0.80 & 16.5 \\
\hline HD 18409 & O9.7 Ib & -5.58 & 30.0 & 2.95 & 3.04 & 16.3 & 0.14 & 150 & 1750 & 5.29 & 10.6 & 1.02 & $0.85^{3)}$ & 15.7 \\
\hline HD 191423 & O9 III:n* & -5.24 & 32.5 & 3.35 & 3.60 & 12.9 & 0.20 & 400 & 1150 & 5.23 & 24.6 & $\leq 0.41$ & 0.80 & 12.7 \\
\hline HD 149757 & $09 \mathrm{~V}$ & -4.35 & 32.0 & 3.65 & 3.85 & 8.9 & 0.17 & 400 & 1550 & 4.87 & 20.2 & $\leq 0.18$ & 0.80 & 8.2 \\
\hline
\end{tabular}

1) Component of binary system.

2) $\log g$ including centrifugal correction (see text).

3) Denotes those absorption profiles for which there are indications that $\beta$ differs from 0.80 (see text).

regard these values as superior to the "older" ones. However, we additionally provide stellar radii calculated from the "old"

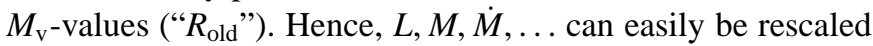
(e.g., Sect. 7.5), accounting for the fact that a strictly differential comparison with earlier analyses is one of the primary objectives of the present work.

Projected rotational velocities. As a first guess we have used the values provided in Paper I (except for HD $210839=\lambda$ Cep, where the value given (i.e., $100 \mathrm{~km} \mathrm{~s}^{-1}$ ) is a miss-print and should read $200 \mathrm{~km} \mathrm{~s}^{-1}$ ). However, in 9 out of 24 cases our analysis (including additional $\mathrm{He}$ I lines) indicated somewhat different values, which we used instead of the original ones. Except for the two stars in Carina, HD 93250 and HD 303308, where we had to increase $V_{\mathrm{r}} \sin i$ from 100 to $130 \mathrm{~km} \mathrm{~s}^{-1}$, these corrections are below 20\%. Compared to the elaborate analyses by Penny (1996) and Howarth et al. (1997) using crosscorrelation techniques based on UV observations, our results agree very well in most cases. With respect to the values presented by Penny (1996) we find an average ratio of the derived projected rotational velocities of $1.03 \pm 0.10$ (mean absolute deviation: \pm 0.08 ), and with respect to the values from Howarth et al. (1997) an analogue comparison results in $1.02 \pm 0.15$ (mean absolute deviation: \pm 0.11 ). The only real difference has been found in the case of HD 93129A, for which Howarth et al. (1997) claim a value of $180 \mathrm{~km} \mathrm{~s}^{-1}$, compared to $130 \mathrm{~km} \mathrm{~s}^{-1}$ in this work. Note, however, that the value provided by Penny (1996), $143 \mathrm{~km} \mathrm{~s}^{-1}$, agrees much better with our analysis for this star.

Mass-loss rates have exclusively been derived from $\mathrm{H}_{\alpha}$. In so far, the consistency (present or absent) of the synthetic and observed He II 4686 line allows to check the accuracy of our code (see below).

Velocity law for thin winds. In case of thin winds, i.e., $\mathrm{H}_{\alpha}$ in absorption, it is (almost) impossible to derive the exponent of the velocity-law, $\beta$, in the wind. In this case, we usually adopted the "theoretical" value $\beta=0.8$ (cf. Pauldrach et al. 1986), but performed a rigorous error analysis concerning the 
possibility that other values are present (cf. Sect. 6). For some stars with $\mathrm{H}_{\alpha}$ in absorption, we actually found indications of values different from $\beta=0.8$. These special cases are described in our comments on individual objects (Sect. 5) and also indicated in Table 1 - the summarized results of our analysis.

Summary of results. This table comprises the HD number, spectral classification, "new" absolute magnitude, effective temperature $T_{\text {eff }}$, "measured" gravity $\log g$, "true" gravity $\log g_{\text {true }}$ (including the centrifugal correction, cf. Sect. 6), stellar radius $R_{*}$ (see above), the luminosity $L$, the helium abundance (by number) $Y_{\mathrm{He}}=N(\mathrm{He}) / N(\mathrm{H})$, projected rotation velocity $V_{\mathrm{r}} \sin i$, terminal velocity $v_{\infty}$, mass $M_{*}$, mass-loss rate $\dot{M}$ and, as mentioned above, the derived or adopted value of $\beta$. Furthermore, we provide also the stellar radii as calculated from the $M_{\mathrm{v}}$-values from Paper I, $R_{\text {old }}$.

The spectral classification used is the one adopted by Herrero et al. (1992) except for those objects for which a re-classification of luminosity class has been proposed. The main purpose of this re-classification was to reduce the scatter of physical parameters (e.g., gravity) within a given luminosity class and to adopt consistent absolute magnitudes. Since in the present work we make no use of any parameters calibrated against luminosity class (except for the absolute magnitude of $\alpha \mathrm{Cam}$ ), a re-classification is not necessary. Therefore, we prefer to maintain the classification based on purely morphological aspects. Note, however, that some stars might have physical parameters that deviate from those obtained using calibrations.

Moreover, according to Walborn et al. (2002), HD 93129A and HD 303308 (prior to knowing that the two stars were binaries; see Nelan et al. 2003, in prep.) have been revised to O2 If* and $\mathrm{O} 4 \mathrm{~V}\left(\left(\mathrm{f}^{+}\right)\right)$, respectively.

The final fits for our sample stars are plotted in Figs. 1 to 7 . Figures 1,2, 5 and 8 display the fits of those lines which are preferentially formed in the photosphere, whereas Figs. 3, 4, 6 and 7 comprise the "wind lines", $\mathrm{H}_{\alpha}$ (along with He II 6527) and He II 4686.

Strategic lines. For the photospheric lines we display the hydrogen Balmer lines $\mathrm{H}_{\beta}$ and $\mathrm{H}_{\gamma}\left(\mathrm{H}_{\delta}\right.$ and $\mathrm{H}_{\epsilon}$ are absent in most of our spectra since they lie at the far edge of the short wavelength range), the He I singlets $\lambda \lambda 4387,4922$, the He I triplets $\lambda \lambda$ 4471, 4713 (He I 4026 again is absent in most cases) and the He II lines $\lambda \lambda 4200,4541$. Additionally, we have included those He lines neighboring $\mathrm{H}_{\alpha}$, namely He II 6404 and He II 6683/He I 6678.

In former analyses mainly two He II lines, He II $\lambda \lambda 4200$ and $4541(n=4 \rightarrow 11$ and $n=4 \rightarrow 9)$ have been used to derive the stellar parameters, since He II 4686 , on many occasions, is affected by severe wind emission which could not be synthesized from plane-parallel models. Moreover, He II 4686 depends strongly on the behaviour of the He II resonance line at $303 \AA$, which in turn reacts sensitively to the details of lineblocking (as all other He II resonance lines do).

Since the present code can deal with both winds and lineblocking, this line has now been included and serves as an ideal tool to indirectly check the accuracy of the calculated lineblocking in the EUV.

Moreover, as already mentioned, we have included the He lines located blue- and redwards of $\mathrm{H}_{\alpha}$ into our analysis, providing additional constraints and information on the sensitivity to small parametric changes and thus allowing to check the consistency of our assumptions and results. In particular, we added the two He II lines at $6404 \AA$ and $6527 \AA$ bluewards of $\mathrm{H}_{\alpha}$ with corresponding transitions $n=5 \rightarrow 15$ and $n=5 \rightarrow 14$, respectively. Redwards of $\mathrm{H}_{\alpha}$ we included He II $6683(n=5 \rightarrow 13)$ which is blended with He I 6678. The latter line belongs to the singlet system with lower level $\left(2 \mathrm{p}^{1} \mathrm{P}^{0}\right)$ and upper level $\left(3 d^{1} D\right)$.

Before beginning to comment on the individual objects, we would like to point out some general behaviour of the fitted lines.

Line cores of photospheric Balmer lines. For almost all luminosity class I objects from our sample with $T_{\text {eff }}>35000 \mathrm{~K}$, the synthetic Balmer lines formed in or close to the photosphere $\left(\mathrm{H}_{\gamma}\right.$ and $\mathrm{H}_{\delta}$, where present, along with $\mathrm{H}_{\beta}$ ) show too much wind emission in their cores if $\mathrm{H}_{\alpha}$ fits (cf. Fig. 1, in particular $\zeta$ Pup). In these cases, it turned out that it is impossible (within the standard assumptions of our model) to obtain a consistent fit for all Balmer lines at a given mass-loss rate. This finding, however, is not completely new, cf. Herrero et al. (2000, 2002). Reversing our modus operandi we obtain well fitted line cores but rather poor $\mathrm{H}_{\alpha}$ profiles if we reduce $\dot{M}$ by a factor of typically $1.5-2$.

On the other hand, for those supergiants with $T_{\text {eff }} \leq$ $35000 \mathrm{~K}$ we either obtain a good fit quality for all Balmer lines or (in two cases) $\mathrm{H}_{\gamma}$ and/or $\mathrm{H}_{\beta}$ show too little wind emission in their cores.

"Generalized dilution effect". Another prominent feature found in class I to III objects is the presence of the so-called "generalized dilution effect".

"Historically", this effect expresses the strengthening of the He I absorption lines with decreasing effective temperature (see Voels et al. 1989 and references therein) and has been invoked to explain certain deviations between synthetic line profiles from plane-parallel models and observations in cool O-supergiants: in this spectral range, one usually finds that a number of synthetic He I lines are considerably weaker than the observations, whereas this effect is most prominent for He I 4471.

The conventional explanation assumes that the lower levels of the corresponding transitions, $2^{3} \mathrm{~S}, 2^{1} \mathrm{~S}, 2^{3} \mathrm{P}$, and $2^{1} \mathrm{P}$ become overpopulated (with decreasing degree of overpopulation) because of the dilution of the radiation field in the (lower) wind. Note that the NLTE departure coefficients scale with the inverse of the dilution factor, since the ionization rates are proportional 
to this quantity (less ionization from a diluted radiation field), whereas the recombination rates remain unaffected.

Once more, this explanation is based on principal theoretical considerations, without any direct proof by actual simulations accounting for an extended atmosphere.

From the results of our simulations (which now include such a treatment), however, it is obvious that there still might be something missing in the above interpretation. In particular He I 4471 is still too weak in cooler supergiants, even if we account for a significant micro-turbulence (see above). Again, this finding is supported by previous investigations from Herrero et al. $(2000,2002)$.

Another consequence of the above theoretical scenario would be the following: For each of the lower He I levels under consideration, the lines belonging to one series should become less affected by the dilution of the radiation field with decreasing oscillator strength, since the line is formed at increasingly greater depths.

This would imply, e.g., that He I 6678 with lower level $2^{1} \mathrm{P}$ (larger oscillator strength but less overpopulated lower level) should approximately be as strongly affected by dilution as He I 4471 (with lower level $2^{3} \mathrm{P}$ ). From our results, however, we can see that also this prediction does not hold if checked by simulations. A typical example is $\alpha$ Cam: Although He I 4471 is too weak, He I 6678 can perfectly be fitted.

At least for all other lines investigated, the prediction seems to hold. The weakest transitions in each series, i.e., the He I 4713 triplet line and the He I 4387 singlet line, give very good line fits and the same is true for He I 4922.

Hence, the only line with prominent generalized dilution effect (we keep this denotation) is He I 4471 and cannot be reproduced by our code even if line-blocking/blanketing is included. Similarly, it is rather improbable that a too large wind emission in the line core (as found for the blue Balmer lines) is the reason for this "defect", since this problems seems to be present only in hotter supergiants. For the cooler ones, where He I 4471 is too weak, the line cores of all other lines are equally well described.

Thus, the actual origin of the dilution effect in He I 4471 is unclear, although a tight relation to either luminosity and/or the presence of a (strong) wind seems to be obvious: dwarfs do not suffer from this effect, no matter if early or late type dwarfs, as can be seen from the almost perfect fit quality of He I 4471 in these cases (Fig. 8).

On the other hand, all O-type class I and III objects between O6 and O9.5 show too weak He I 4471, whereas stars earlier than $\mathrm{O} 6$ behave like class $\mathrm{V}$ objects, i.e., they pose no problem.

The boundary for the onset of the dilution effect, however, is difficult to determine. Our model calculations of HD 210839 (O6 I(n) fp) which constitutes an upper boundary for the effect in class I objects reveal that a decrease in $T_{\text {eff }}$ or $\beta$ along with corresponding changes in $\dot{M}$ helps to improve the $\mathrm{H}_{\gamma}, \mathrm{H}_{\beta}$ and He I 4471 line fits, whereas the good fit quality for the other lines is lost in this case. The situation is similar for HD 190864 (O6.5 III(f)). No matter which sensible parametric alterations we applied, there were hardly any changes in He I 4471.
From these experiments, we estimate the upper boundary for the presence of the dilution effect to lie somewhere between $\mathrm{O} 6$ and $\mathrm{O} 6.5$ for class I and III objects.

It cannot be excluded, of course, that the discussed effect is a deficiency of the present version of FASTWIND. Combined UV/optical CMFGEN analyses by Crowther et al. (2002) and Hillier et al. (2003) for LMC/SMC supergiants do actually reproduce the strength of He I 4471 in parallel with the other lines, but the number of objects analyzed is still too low to allow for firm conclusions. Nevertheless, we are aware of the fact that a consistent calculation of the temperature structure (also in the outer wind) might be relevant for the formation of the He I 4471 line cores, particularly in the parameter space under consideration; since a new version of FASTWIND will include such a consistent temperature stratification, we will be able to report on any changes due to this improvement in forthcoming publications.

\section{Comments on individual objects}

In the following section we will give specific comments on peculiarities, problems and uncertainties for each individual object, starting with the hottest of each luminosity class and ordered according to derived $T_{\text {eff }}$.

\subsection{Supergiants}

HD 93129A. The re-analysis of this object reveals a $T_{\text {eff }}$ of $42500 \mathrm{~K}$ (compared to $T_{\text {eff }}=50500 \mathrm{~K}$ from Paper I) which constitutes the most significant change in $T_{\text {eff }}$ found throughout the course of this investigation. The upper temperature limit lies at $45000 \mathrm{~K}$ where the wings in the He II lines start to become too strong. Before a final statement concerning the effective temperature can be given, the nitrogen spectrum will have to be synthesized, of course.

The value of $\beta$ has been constrained to 0.8 and the helium abundance to $Y_{\mathrm{He}}=0.1$. A larger helium abundance can be excluded since an increase in $Y_{\mathrm{He}}$ would yield too strong absorption troughs. The reader may note that this object was recently confirmed as a binary with a separation of 60 mas (Nelan et al. 2003 , in prep.), where the components have been found to be similar with respect to their spectral types and masses. Thus, the observed spectrum might be significantly contaminated and the results of our analysis are somewhat artificial (especially concerning all radius dependent quantities such as mass, luminosity and mass-loss rate. If we assume that both components were actually identical, the values for radius, luminosity, mass and mass-loss rate given in Table 1 would have to be scaled by a factor of $2^{-1 / 2}, 1 / 2,1 / 2$ and $2^{-3 / 4}$, respectively, in order to obtain the corresponding values for one component.) Note, however, that the deduced reduction in $T_{\text {eff }}$ (as a consequence of severe line-blanketing) sounds reasonable and gives some clue about what would happen if the object were a single star.

Since the value for $V_{\mathrm{r}} \sin i$ claimed by Howarth et al. (1997) significantly exceeds the value deduced by us (cf. Sect. 4), we have also determined an upper limit for this value. In order to obtain synthetic spectra consistent with the observations, this 
HD_NR $\quad \mathrm{H} \gamma \quad \mathrm{H} \beta \quad$ HeI4387 Hel4922 $\underset{\text { HeII6683 }}{\operatorname{Hel6678}}$ HeI4471 Hel4713 HeII4200 HeII4541 Hell6404

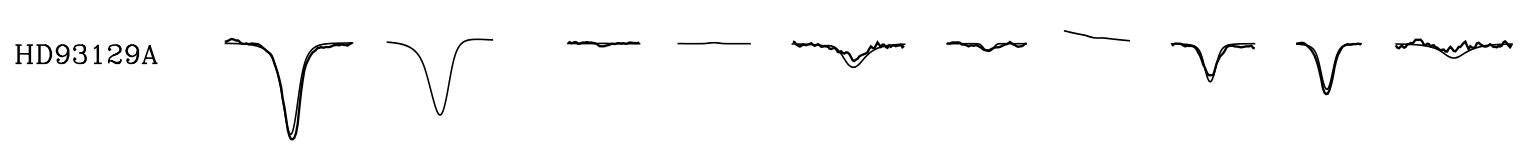

HD66811
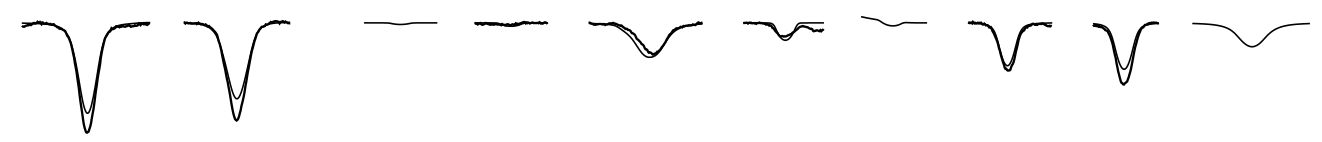

HD14947

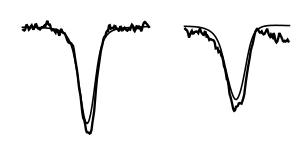

ind

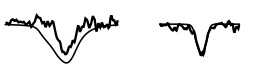

mone

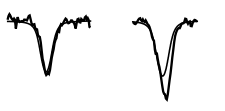

4h

HD210839

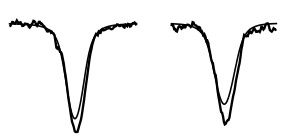

man

nun on

$\sqrt{N^{2}}$

Thant
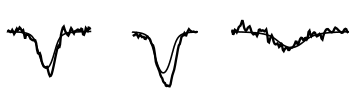

HD 192639

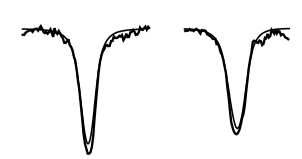

rim<smiles>CCCCCCCCCCCCCCCCCCC</smiles>

$\sqrt{2}$

Fig. 1. Line fits of supergiants with spectral types ranging from $\mathrm{O} 3$ to $\mathrm{O} 7.5$, ordered according to derived $T_{\text {eff. }}$ The horizontal and vertical lines in the bottom right corner indicate the scale used and correspond to $20 \AA$ in wavelength and 0.5 in units of the continuum, respectively (extending from 0.75 to 1.25 ).
HD_NR
$\mathrm{H} \gamma \quad \mathrm{H} \beta$
HeI4387 HeI4922 HeI6678
HeI4471 HeI4713 HeII4200 HeII454 1 HeII6404 HeII6683

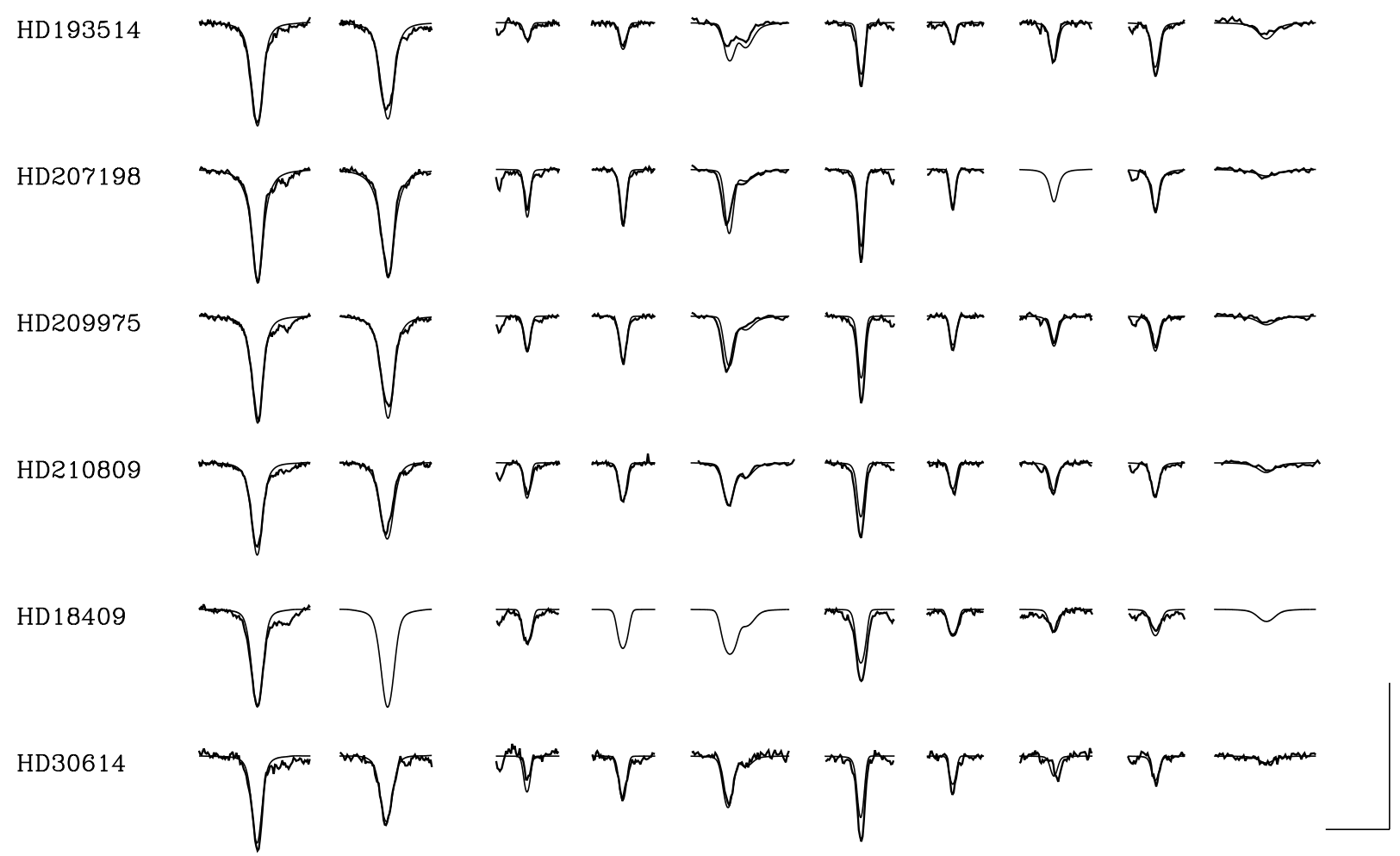

Fig. 2. As Fig. 1, but for spectral types ranging from $\mathrm{O} 7$ to 09.7 . 
HD_NR

HD93129A

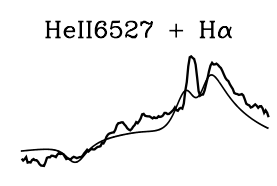

HeII4686

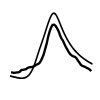

HD66811
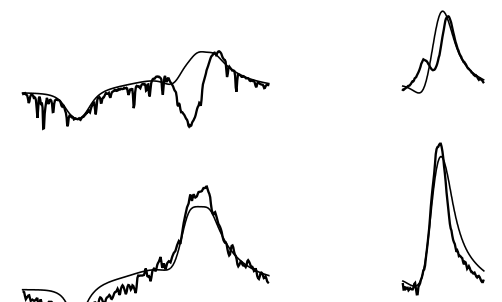

HD 14947

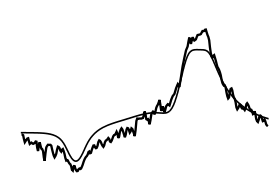

HD2 10839

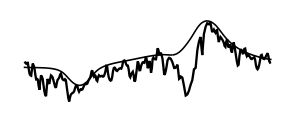

HD192639

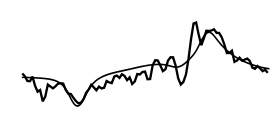

A

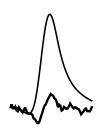

HD_NR

HD 193514

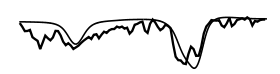

HD207198

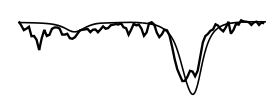

HD209975

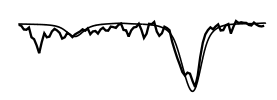

HD210809

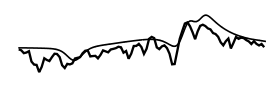

HD18409

HD30614

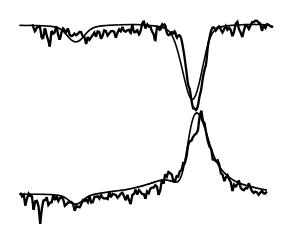

HeII4686

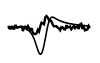

Fig. 3. "Wind lines" of the hotter supergiants in Fig. 1.

limit turned out to be $150 \mathrm{~km} \mathrm{~s}^{-1}$, very close to the alternative value provided by Penny (1996).

HD 66811 ( $\zeta$ Pup). For this star, as already discussed, the line cores of $\mathrm{H}_{\gamma}$ and $\mathrm{H}_{\beta}$ become too strongly filled in by wind emission if we use the mass-loss rate derived from a fit of $\mathrm{H}_{\alpha}$. In this case, we have concentrated on the red wing of $\mathrm{H}_{\alpha}$ since the blue wing is known to be problematic (see also Paper I). In particular, the (strong) blue absorption trough cannot be reproduced by our models. It might be speculated whether this feature is related to an aspherical wind which should be present because of the large value of $V_{\mathrm{r}} \sin i$ and which is supported by spectropolarimetric analyses carried out by Harries \& Howarth (1996).

Compared to the results from Paper I, $\dot{M}$ needed to be increased from 6.0 to $8.8 \times 10^{-6} M_{\odot} / \mathrm{yr}$, mainly because $\beta$ had to be reduced from 1.15 to 0.90 .

A lower limit for the mass-loss rate of $7.4 \times 10^{-6} M_{\odot} / \mathrm{yr}$ can be inferred if we try to reproduce the line cores of $\mathrm{H}_{\gamma}, \mathrm{H}_{\beta}$ and $\mathrm{He}$ II 4541; in this case, $\mathrm{H}_{\alpha}$ and He II 4686 become much too weak, of course. From these limits, however, it might be possible to derive tight constraints concerning the possibility of wind clumping (see Sect. 7.5.2).

Although the fit quality for He II 4200 is good, He II 4541 (with same lower level) appears too weak. The discrepancy between these two lines (which is evident also for the next two stars, HD 14947 and $\lambda$ Cep) has already been discussed by Herrero et al. (1992, 2000) for plane-parallel and unified model atmospheres without line-blocking/blanketing,
Fig. 4. "Wind lines" of the cooler supergiants in Fig. 2.

respectively. The inclusion of the latter effects does not resolve the problem. Interestingly, it seems to occur only in those cases where the line cores of $\mathrm{H}_{\gamma}$ and $\mathrm{H}_{\beta}$ are too weak.

HD 14947. The overall fit quality is good, but again no optimum solution for the line cores of the blue Balmer lines could be obtained. In order to match the profile shape of He II 4686, $\beta$ had to be increased by 0.25 and $\dot{M}$ to be decreased by $25 \%$ (from $8.5 \times 10^{-6} M_{\odot} / \mathrm{yr}$ to $6.4 \times 10^{-6} M_{\odot} / \mathrm{yr}$ ), compared to the values derived from $\mathrm{H}_{\alpha}$.

Since He I 4471 is the only He I line with considerable strength, the ionization equilibrium (and thus the effective temperature) remains somewhat uncertain, due to missing additional constraints.

The apparent discrepancy between the predicted and observed line profile of He II 6683 is partly due to an erroneous rectification.

HD210839 ( $\lambda$ Cep). This star, as $\zeta$ Pup, is known to be a fast rotator with a projected rotational speed of $V_{\mathrm{r}} \sin i=200 \mathrm{~km} \mathrm{~s}^{-1}$. Also the fit quality is very similar to $\zeta$ Pup: We find the same line core problems in $\mathrm{H}_{\gamma}$ and $\mathrm{H}_{\beta}$ and no possibility to obtain the observed $\mathrm{P}$ Cygni shape in $\mathrm{H}_{\alpha}$ (again aspherical wind?). Note that HD 210839 is the first star with observable dilution effect. Note also that the rather large uncertainty in $M_{\mathrm{v}}$ (due to the distance problem discussed in Sect. 4) leads to correspondingly large error bars in $\log L, \dot{M}$ and related quantities. 


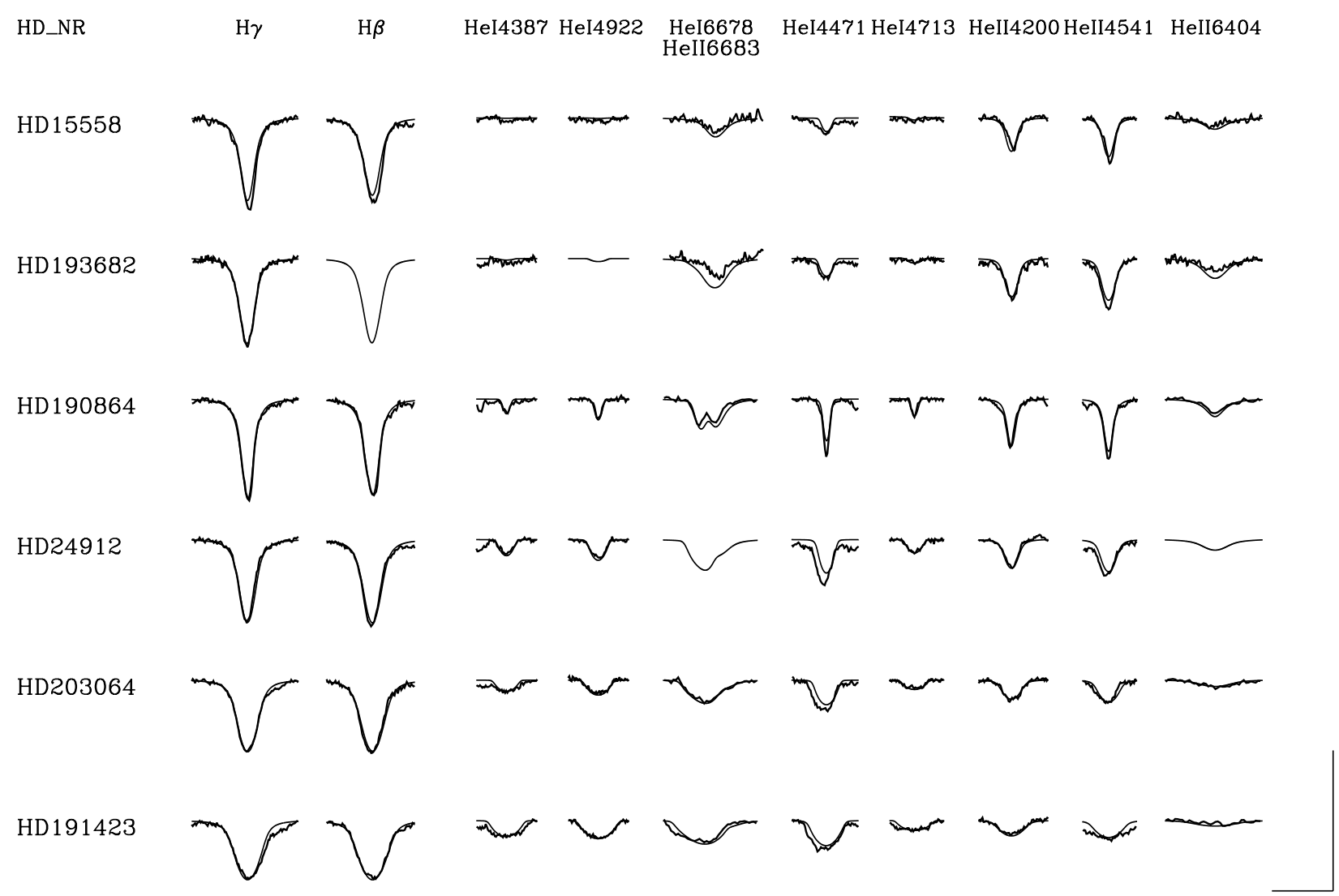

Fig. 5. Line fits of the giant sample with spectral types ranging from $\mathrm{O} 5$ to $\mathrm{O} 9$, ordered according to derived $T_{\text {eff }}$.

HD 192639. Our re-analysis gives a rather consistent fit of both the Balmer lines and the weak He I lines, with exception of the strong dilution effect observed in He I 4471.

For this star, we found the most striking discrepancy between theoretical prediction and observation in He II 4686, where theory predicts strong emission but a weak P Cygni shaped profile is observed instead. In order to fit this line appropriately, it would be necessary to decrease the massloss rate by more than $50 \%$ (from $\dot{M}=6.3 \times 10^{-6} M_{\odot} /$ yr to $\dot{M} \approx 2.8 \times 10^{-6} M_{\odot} /$ yr.) Note that this star has parameters and profiles similar to $\lambda$ Cep. The latter is known to be strongly variable (cf. Herrero et al. 2000) and, thus, it might be possible that also for HD 192639 the apparent mismatch of $\mathrm{H}_{\alpha}$ and He II 4686 might be partly related to wind variability: As pointed out in Sect. 3, the blue and red spectra have not been taken simultaneously, but with a temporal offset larger than the typical wind flow time which is of the order of a couple of hours.

The apparent bad fit of He II 6404 is solely due to an erroneous rectification.

HD 193514. The presence of a wind is evident from He II 4686 showing a weak emission. By comparing the star to HD 192639 which is of same spectral type with similar values for $T_{\text {eff }}$ and $\log g$, we can see that the $\mathrm{H}_{\alpha}$ line in the case of HD 193514 is in absorption, whereas in the case of HD 192639 it is in emission. Accordingly, the derived mass-loss rate for HD 193514 has half the value of HD 192639.
HD 207198. The fit quality for this sample star is very good (except for a small dilution effect in He I 4471), so no further comment is necessary.

HD 209975: The spectrum and line fit is very similar to HD 207198 and also the parameters deduced lie close together. Compared to HD 207198, this star has a slightly lower $T_{\text {eff }}$ along with a 0.25 dex lower value for $\log g$. Note that the dilution effect in He I 4471 is considerably stronger.

HD 210809. For this star, the synthetic $\mathrm{H}_{\gamma}$ and $\mathrm{H}_{\beta}$ profiles are slightly too strong in absorption, in contrast to all cases encountered so far.

He II 4686 reveals a huge difference between theoretical prediction and observation. The theoretical emission feature as shown in Fig. 4 is similar to the one observed in HD 192639 (but not as prominent). In this temperature range, the line reacts strongly to small changes in temperature. Around a critical temperature of $T_{\text {eff }}=30000 \mathrm{~K}$, He II 4686 switches from absorption to emission, i.e., at that temperature we would be able to fit the line perfectly. Nevertheless, we have retained the higher value $(31500 \mathrm{~K})$ since this value gives a more consistent fit concerning the remaining lines. This discrepancy which points to some possible problems in our treatment of lineblocking around $303 \AA$ (or could be also related to wind variability) will be accounted for in our error analysis when discussing the error bars for $T_{\text {eff }}$. 


HD203064

Fig. 6. "Wind lines" of the giants in Fig. 5.

HD 18409. This star comprises a similar problem as found in HD 203064 and $\xi$ Per (see below): The photospheric value of $V_{\mathrm{r}} \sin i=150 \mathrm{~km} \mathrm{~s}^{-1}$ has to be reduced to $V_{\mathrm{r}} \sin i=80 \mathrm{~km} \mathrm{~s}^{-1}$ in the case of $\mathrm{H}_{\alpha}$. Although this line is in absorption, we favor a value of $\beta=0.85$ which improves the fit quality of the wings of $\mathrm{H}_{\alpha}$ and $\mathrm{He}$ II 4686 moderately.

HD 30614 ( $\alpha$ Cam). The effects of line blocking on $T_{\text {eff }}$ are rather weak, and in order to obtain a convincing fit we had to decrease the helium abundance from $Y_{\mathrm{He}}=0.2$ to $Y_{\mathrm{He}}=0.10$. $\beta$ turned out to be slightly larger than derived in Paper I (1.15 compared to 1.10). Note that the value derived for $T_{\text {eff }}$, $29000 \mathrm{~K}$, is identical to the value obtained via a UV-analysis performed by Pauldrach et al. (2001, WMbasic).

\subsection{Giants}

HD 15558. The line fits obtained are in good agreement with the observations and especially the Balmer lines give a consistent fit. $T_{\text {eff }}$ was reduced by $\approx 13 \%$ to $41000 \mathrm{~K}$, whereas the other parameters remained more or less at their old values (except for the rotational velocity which had to be adapted from $120 \mathrm{~km} \mathrm{~s}^{-1}$ to $150 \mathrm{~km} \mathrm{~s}^{-1}$ ).

The rather small discrepancy between theoretical prediction and observation in the case of He II 4686 can be removed by increasing $\dot{M}$ from $5.6 \times 10^{-6} M_{\odot} /$ yr to $6.5 \times 10^{-6} M_{\odot} /$ yr.

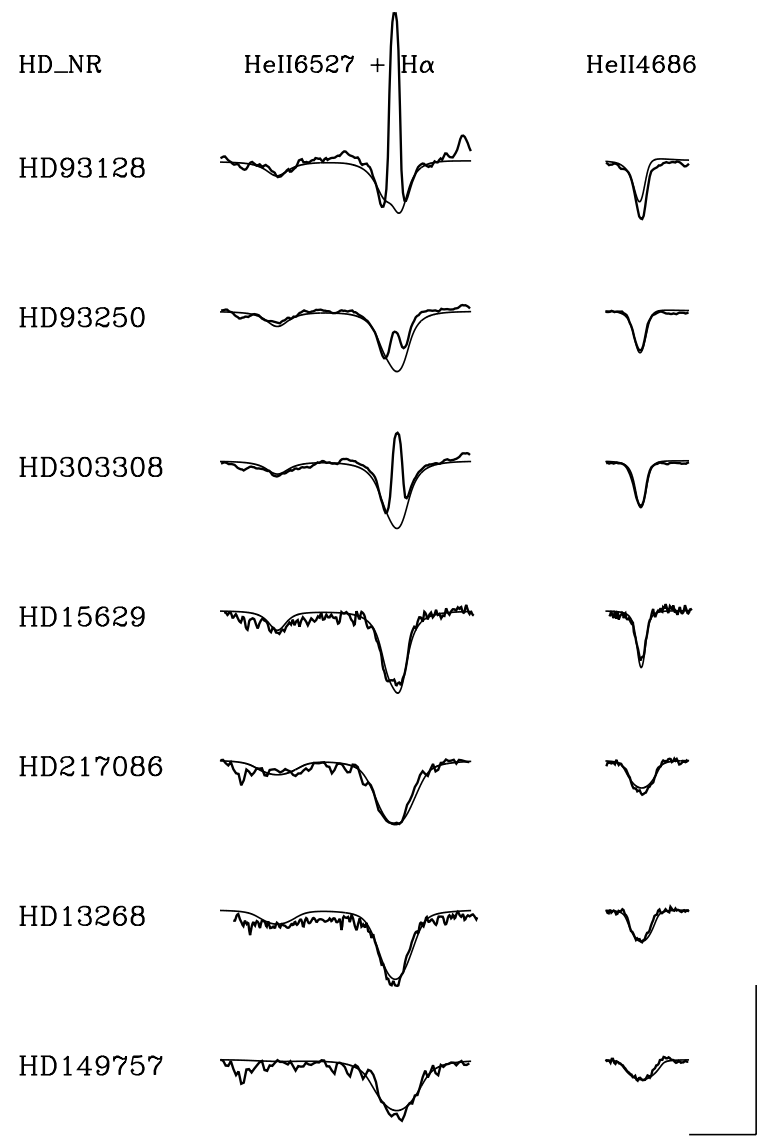

Fig. 7. "Wind lines" of the dwarfs in Fig. 8.

HD 193682. As can be seen in Fig. 6, the red wings of $\mathrm{H}_{\alpha}$ and of He II 4686 do not fit the observations perfectly, but constitute the best compromise concerning the overall fit quality of the spectrum.

The rotational speed $V_{\mathrm{r}} \sin i$ was found to be $200 \mathrm{~km} \mathrm{~s}^{-1}$, although with a value of $180 \mathrm{~km} \mathrm{~s}^{-1}$ an improved fit quality of the $\mathrm{H}_{\alpha}$ line could be achieved.

Compared to the values from Paper I (which relied on the analysis by Herrero et al. 1992), the helium abundance, $Y_{\mathrm{He}}$, needed to be drastically decreased, from 0.43 to 0.20 . This reduction (obtained by requiring a comparable fit quality for all lines) is mainly a consequence of the reduction of $T_{\text {eff }}$ by $5000 \mathrm{~K}$ and the inclusion of the additional He lines in our analysis as described above.

HD 190864. The re-analysis gives a consistent fit for the Balmer lines and all $\mathrm{He}$ I and He II lines with exception of the dilution effect observed in He I 4471. Although $T_{\text {eff }}$ had to be reduced by $4000 \mathrm{~K}$ to $37000 \mathrm{~K}, \log g$ remained at its old value. Again, the helium abundance needed to be decreased, in this case from 0.2 to 0.15 . A value of $\beta=0.8$ was already suggested in Paper I, and also the differences in the derived massloss rates are negligible.

HD203064 is an extremely rapid rotator with $V_{\mathrm{r}} \sin i=300 \mathrm{~km} \mathrm{~s}^{-1}$ which is clearly visible in the broadened line profiles as shown in Fig. 5. The theoretical predictions 


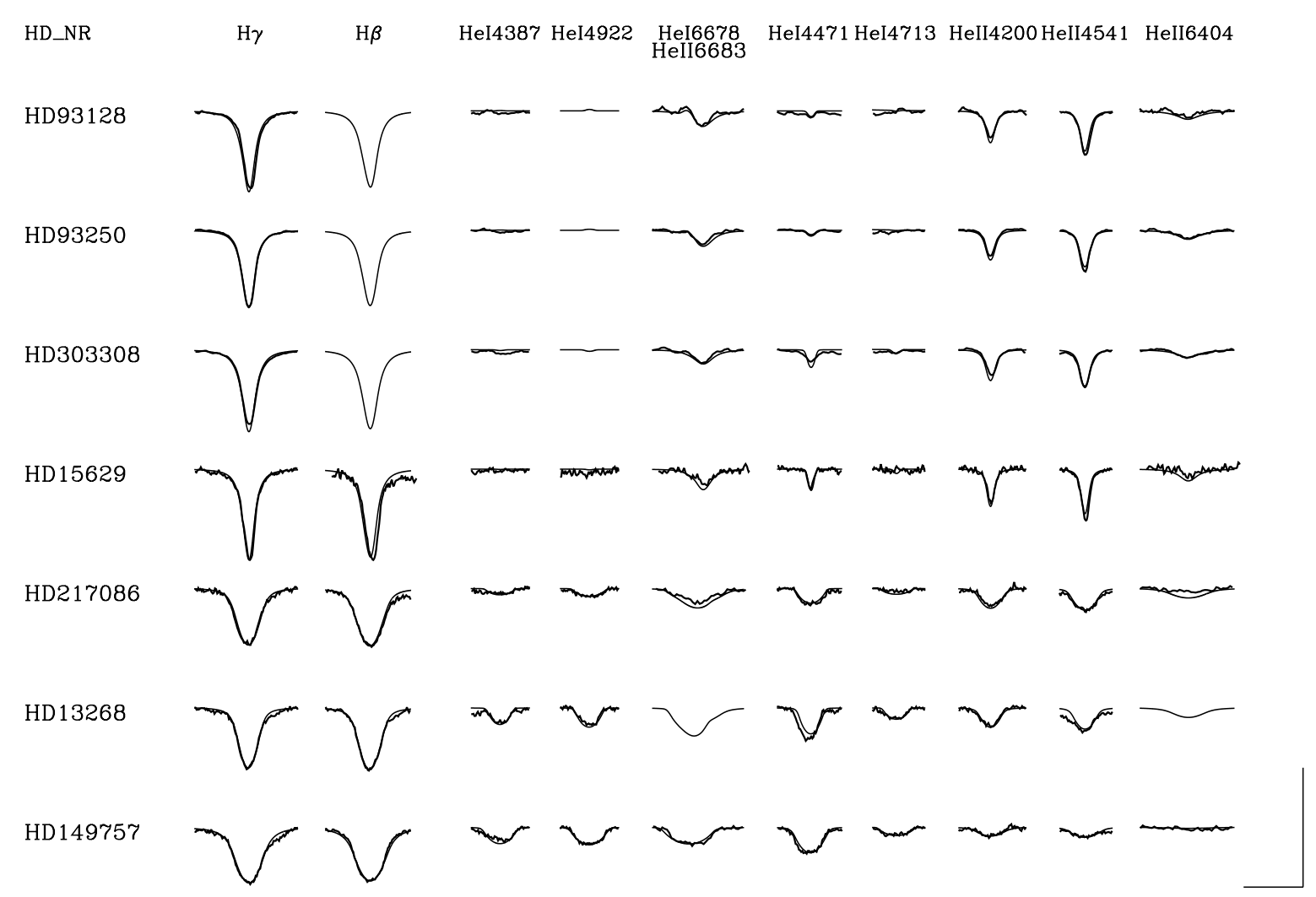

Fig. 8. Line fits of the dwarf sample with spectral types ranging from $\mathrm{O} 3$ to $\mathrm{O} 9$, ordered according to derived $T_{\text {eff }}$.

agree well with the observations apart from the dilution effect in He I 4471.

The star behaves prototypical for a number of giants (and the supergiant HD 18409) with large values of $V_{\mathrm{r}} \sin i$ : Whereas $\mathrm{H}_{\gamma}$ and $\mathrm{H}_{\beta}$ reveal a consistent fit, only the line cores of $\mathrm{H}_{\alpha}$ and $\mathrm{He}$ II 4686 are in agreement with the observations. The wings of both lines, however, are too narrow compared to the photospheric rotational speed and would be much more consistent if we used a lower value of $190 \mathrm{~km} \mathrm{~s}^{-1}$ (cf. Paper I and Sect. 8).

HD 24912 ( $\xi$ Per). The fit quality is good, with the exception of He I 4471 which apart from the generalized dilution effect also comprises a small error in rectification. Photospheric lines display a projected rotational speed of $V_{\mathrm{r}} \sin i=220 \mathrm{~km} \mathrm{~s}^{-1}$, while $\mathrm{H}_{\alpha}$ indicates a much lower value, $\$ 100 \mathrm{~km} \mathrm{~s}^{-1}$ (for further comments see Paper I).

HD 191423 together with HD 149757 are the fastest rotators in the whole sample with a projected rotational speed of $V_{\mathrm{r}} \sin i=400 \mathrm{~km} \mathrm{~s}^{-1}$. As for the previous two stars, the wings of $\mathrm{H}_{\alpha}$ are too narrow compared to $\mathrm{H}_{\gamma}$ and $\mathrm{H}_{\beta}$, corresponding to an "effective" value of $300 \mathrm{~km} \mathrm{~s}^{-1}$.

Line blanketing leads to a reduction in $T_{\text {eff }}$ by $1500 \mathrm{~K}$, and the mass-loss rate had to be increased by nearly a factor of two (from $\dot{M}=0.2 \times 10^{-6} M_{\odot} /$ yr to $\dot{M}=0.4 \times 10^{-6} M_{\odot} / \mathrm{yr}$ ). Note that the profile points to a disk like structure as discussed in Paper I.

The derived helium abundance is larger than the one obtained by Villamariz et al. $\left(2002, Y_{\mathrm{He}}=0.14\right)$. In essence, this difference is mainly due to the lower micro-turbulent velocity adopted by us.

\subsection{Dwarfs}

HD 93128. The $\mathrm{H}_{\alpha}$ line cores of all dwarfs in our sample, which are located in Carina (HD 93128, HD 93250 and HD 303308), are contaminated by nebula emission which makes the determination of $\dot{M}$ a somewhat difficult task. For HD 93128, we derive a mass-loss rate of $2.65 \times 10^{-6} M_{\odot} / \mathrm{yr}$ which is roughly a factor of two higher than the value obtained in Paper I. This value is an upper limit (the lower one is given in the next section) and has been used to calculate the final model. Line-blanketing leads to a decrease in $T_{\text {eff }}$ from $52000 \mathrm{~K}$ to $46500 \mathrm{~K}$ without changing $\log g$. With $\beta=0.85$ we were able to improve the fit of the wings of $\mathrm{H}_{\alpha}$, although this procedure turned out to be rather difficult due to the contamination by nebula emission.

HD 93250. The profiles are generally very similar to HD 93128, although HD 93250 seems to be less affected by line-blanketing effects. We had to reduce $T_{\text {eff }}$ to $46000 \mathrm{~K}$ (from the older value of $50500 \mathrm{~K}$ ), again with no changes in $\log g . \dot{M}$ is decreased by $1.4 \times 10^{-6} M_{\odot} / \mathrm{yr}$ to $3.5 \times 10^{-6} M_{\odot} / \mathrm{yr}$, for a value of $\beta=0.90$ which resulted from a compromise between the fit of the line core and the wings of both $\mathrm{H}_{\alpha}$ and He II 4686 .

HD 303308. For this sample star we had to apply a relatively large $T_{\text {eff-correction of }} 7000 \mathrm{~K}$ (to $41000 \mathrm{~K}$ ) along 
with a change in $\log g$ of 0.15 dex (to 3.90$). \dot{M}$ is slightly reduced, whereas the rotational speed had to be increased from $100 \mathrm{~km} \mathrm{~s}^{-1}$ to $120 \mathrm{~km} \mathrm{~s}^{-1}$. Interestingly, our model calculations display an under-abundance in helium, $Y_{\mathrm{He}}=0.075$. The star is a "very likely" binary with a separation of approximately 14 mas (Nelan et al. 2003, in prep.), implying that the results might be somewhat artificial. However, from the rather good fit quality of the profiles, the contamination brought about by the companion seems to be negligible.

HD 15629. For this star, $T_{\text {eff }}$ needed to be decreased quite drastically, from $47000 \mathrm{~K}$ to $40500 \mathrm{~K}$ with an appropriate adjustment of $\log g$ to 3.70. The mass-loss rate is moderate $\left(\dot{M}=1.3 \times 10^{-6} M_{\odot} /\right.$ yr) but almost twice as high as deduced in Paper I. The fit quality is generally good, and we confirm the helium deficiency to be $Y_{\mathrm{He}}=0.08$ as found in Paper I and by Herrero et al. (2000).

HD 217086. For both the Balmer and the He I lines we obtain a very good fit quality, but there are still small discrepancies for He II 6683 and He II 6404 which constitutes a problem in other sample stars as well. However, usually they occur only in stars with low rotational speed. HD 217086 is a fast rotator with $V_{\mathrm{r}} \sin i=350 \mathrm{~km} \mathrm{~s}^{-1}$ and exhibits very broad line profiles as shown in Figs. 8 and 7 . According to Paper I there is a difference in the photospheric and the "effective" rotational velocity of $85 \mathrm{~km} \mathrm{~s}^{-1}$ which could not be confirmed in our present analysis. The upper limit for the mass-loss rate was found to be $0.23 \times 10^{-6} M_{\odot} /$ yr for an adopted $\beta=0.80$.

HD 13268. Although being a fast rotator with $V_{\mathrm{r}} \sin i=300 \mathrm{~km} \mathrm{~s}^{-1}$, the photospheric value, again, applies to the $\mathrm{H}_{\alpha}$ profile (in contrast to the findings from Paper I). For the mass-loss rate only an upper limit of $0.26 \times 10^{-6} M_{\odot} / \mathrm{yr}$ can be given, for an adopted value of $\beta=0.80$. The enhanced helium abundance $Y_{\mathrm{He}}=0.25$, as given in Paper I, was retained giving the best compromise especially in the case of He II 4541 which is still slightly too weak.

HD 149757 ( $\zeta$ Oph), finally, is a very fast rotator $\left(V_{\mathrm{r}} \sin i=400 \mathrm{~km} \mathrm{~s}^{-1}\right)$, but also here the photospheric lines and $\mathrm{H}_{\alpha}$ display the same broadening, i.e., the discrepancy found in Paper I could not be confirmed. We obtained a massloss rate of $0.18 \times 10^{-6} M_{\odot} / \mathrm{yr}$ as an upper limit. This value was used for our final model calculation and is considerably higher than previously determined, where $\dot{M}$ was found to be $\approx 0.03 \times 10^{-6} M_{\odot} / \mathrm{yr}$. Moreover, the "old" helium abundance of $Y_{\mathrm{He}}=0.19$ could be slightly decreased to 0.17 giving a very good fit quality as displayed in the corresponding figures.

\section{Error analysis}

In the following section we will discuss the errors estimated (and derived) for the parameters given in Table 1 which will be needed for our further analysis.
Table 2. Stars with $H_{\alpha}$ in emission: Errors in stellar and wind parameters given in Table 1. $\Delta T_{\text {eff }}$ in $\mathrm{kK}, \Delta \beta$ adopted as $\pm 0.1, \Delta \log Q_{1}$ is the error in $Q$-value due to uncertainties in $\mathrm{H}_{\alpha}$ line fit, $\Delta \log Q_{2}$ is the error in $Q$-value arising from uncertainties in $T_{\text {eff }}$ and $\Delta \log Q_{t}$ is the total error. All values have to be preceeded by a \pm sign.

\begin{tabular}{lcccc}
\hline \hline Star & $\Delta T_{\text {eff }}$ & $\Delta \log Q_{1}$ & $\Delta \log Q_{2}$ & $\Delta \log Q_{t}$ \\
\hline HD 93129A & 2.5 & 0.02 & .04 & 0.047 \\
HD 66811 & 1.5 & 0.02 & .03 & 0.035 \\
HD 14947 & 1.5 & 0.02 & .03 & 0.036 \\
HD 210839 & 1.5 & 0.04 & .03 & 0.051 \\
HD 192639 & 1.5 & 0.03 & .035 & 0.045 \\
HD 210809 & 2.5 & 0.04 & .065 & 0.075 \\
HD 30614 & 1.0 & 0.04 & .03 & 0.049 \\
\hline
\end{tabular}

Table 3. Stars with $H_{\alpha}$ in absorption: Errors in stellar and wind parameters given in Table 1. Notation and units as in Table 2, except for the adopted uncertainty in $\beta$ and the corresponding uncertainty in $\dot{M}$ (for stellar radii from Table 1, see text). The upper and lower limits of $\dot{M}$ (in units of $10^{-6} M_{\odot} / \mathrm{yr}$ ) correspond to the lower and upper limits of $\beta$, respectively. The listed errors in $T_{\text {eff }}$ and $\log Q_{2}$ (cf. Table 2) have to be preceeded by a \pm sign.

\begin{tabular}{|c|c|c|c|c|c|c|}
\hline Star & $\Delta T_{\text {eff }}$ & $\Delta \beta$ & $\dot{M}_{1}^{-}$ & $\dot{M}_{1}^{+}$ & $\Delta \log Q_{2}$ & $\Delta \log Q_{t}$ \\
\hline HD 93128 & 1.5 & $\begin{array}{l}+0.15 \\
-0.15\end{array}$ & 1.59 & 3.70 & 0.023 & $\begin{array}{l}+0.15 \\
-0.22\end{array}$ \\
\hline HD 93250 & 1.5 & $\begin{array}{l}-0.10 \\
-0.10\end{array}$ & 2.58 & 3.87 & 0.023 & $\begin{array}{l}+0.05 \\
-0.13\end{array}$ \\
\hline HD 303308 & 1.5 & $\begin{array}{l}+0.20 \\
-0.10\end{array}$ & 1.35 & 2.00 & 0.027 & $\begin{array}{l}+0.09 \\
-0.09\end{array}$ \\
\hline HD 15558 & 1.5 & $\begin{array}{l}+0.20 \\
-0.10\end{array}$ & 3.84 & 6.31 & 0.027 & $\begin{array}{l}+0.06 \\
-0.16\end{array}$ \\
\hline HD 193682 & 1.5 & $\begin{array}{l}+0.20 \\
-0.10\end{array}$ & 0.94 & 2.16 & 0.028 & $\begin{array}{l}+0.10 \\
-0.27\end{array}$ \\
\hline HD 15629 & 1.0 & $\begin{array}{l}+0.20 \\
-0.10\end{array}$ & 0.85 & 1.55 & 0.018 & $\begin{array}{l}+0.08 \\
-0.18\end{array}$ \\
\hline HD 190864 & 1.0 & $\begin{array}{l}-0.10 \\
+0.10\end{array}$ & 0.97 & 1.85 & 0.020 & $\begin{array}{l}+0.10 \\
+0.13 \\
-0.16\end{array}$ \\
\hline HD 193514 & 1.5 & $\begin{array}{l}+0.20 \\
-0.10\end{array}$ & 2.90 & 4.16 & 0.033 & $\begin{array}{l}+0.08 \\
-0.09\end{array}$ \\
\hline HD 24912 & 1.0 & $\begin{array}{l}+0.20 \\
-0.10\end{array}$ & 0.74 & 1.29 & 0.022 & $\begin{array}{l}+0.08 \\
-0.17\end{array}$ \\
\hline HD 203064 & 1.0 & $\begin{array}{l}+0.20 \\
-0.10\end{array}$ & 0.94 & 1.77 & 0.022 & $\begin{array}{l}+0.10 \\
-0.18\end{array}$ \\
\hline HD 217086 & 1.0 & $\begin{array}{l}+0.20 \\
-0.10\end{array}$ & $\leq 0.05$ & $\leq 0.33$ & 0.021 & $\begin{array}{l}+0.17 \\
-0.68\end{array}$ \\
\hline HD 13268 & 1.0 & $\begin{array}{l}+0.20 \\
-0.10\end{array}$ & $\leq 0.12$ & $\leq 0.34$ & 0.024 & $\begin{array}{l}-0.00 \\
+0.13 \\
-0.33\end{array}$ \\
\hline HD 207198 & 1.0 & $\begin{array}{l}+0.20 \\
-0.10\end{array}$ & 1.29 & 2.33 & 0.024 & $\begin{array}{l}+0.12 \\
-0.14\end{array}$ \\
\hline HD 209975 & 1.0 & $\begin{array}{l}+0.20 \\
-0.10\end{array}$ & 1.55 & 2.28 & 0.025 & $\begin{array}{l}+0.04 \\
-0.14\end{array}$ \\
\hline HD 18409 & 1.5 & $\begin{array}{l}+0.15 \\
-0.15\end{array}$ & 0.74 & 1.54 & 0.040 & $\begin{array}{l}+0.18 \\
-0.14\end{array}$ \\
\hline HD 191423 & 1.0 & $\begin{array}{l}-0.13 \\
+0.20 \\
-0.10\end{array}$ & $\leq 0.28$ & $\leq 0.46$ & 0.024 & $\begin{array}{l}-0.14 \\
+0.06 \\
-0.17\end{array}$ \\
\hline HD 149757 & 1.0 & $\begin{array}{l}+0.20 \\
-0.10\end{array}$ & $\leq 0.04$ & $\leq 0.25$ & 0.024 & $\begin{array}{l}+0.14 \\
-0.65\end{array}$ \\
\hline
\end{tabular}

\subsection{Stellar parameters (cf. Table 4)}

Effective temperatures. The formal errors in $T_{\text {eff }}$, estimated from the quality of the helium line fits, generally lie between $\pm 1000 \mathrm{~K}$ and $\pm 1500 \mathrm{~K}$ (Tables 2 to 3 , cf. also Herrero et al. 1992, 2002) with two exceptions: The upper temperature limit for HD 93129A (neglecting its binary status) lies at $45000 \mathrm{~K}$, in contrast to the temperature of our final, best-fitting model at $42500 \mathrm{~K}$. Although somewhat artificial, we will not discard this star from our analysis for the sake of completeness and assume $\Delta T_{\text {eff }}$ to be of the order of $\pm 2500 \mathrm{~K}$. The second case with larger error bars in $T_{\text {eff }}$ constitutes HD 210809 for which we also adopt an error of $\pm 2500 \mathrm{~K}$, due to the dilemma concerning He II 4686 (cf. Sect. 5). Since we found the critical temperature, where this line switches from absorption to emission, to be located at $T_{\text {eff }}=30000 \mathrm{~K}$ (compared to $T_{\text {eff }}=31500 \mathrm{~K}$ for 
Table 4. Parameters and corresponding errors for our sample stars. For errors in $T_{\text {eff }}$ and $\log Q$, see Tables 2, 3. All quantities are given in the same units as in Table 1. $D_{\text {mom }}$ denotes the modified wind-momentum rate (Eq. (14)) and is given in cgs-units. Note that all values quoted for HD 93129A and HD 303308 may (strongly) suffer from a possible contamination by a companion. Only the values for $T_{\text {eff }}, \log g, Y_{\mathrm{He}}$ and $Q$ (which are more or less independent of $V$ ) might be considered to be of correct order of magnitude.

\begin{tabular}{|c|c|c|c|c|c|c|c|c|c|c|c|c|c|c|}
\hline Star & $\log g_{\text {true }}$ & $\Delta \log g_{\text {true }}$ & $R_{*}$ & $\Delta R_{*}$ & $Y_{\mathrm{He}}$ & $\Delta Y_{\mathrm{He}}$ & $\log L$ & $\Delta \log L$ & $M_{*}$ & $\Delta M_{*}$ & $\log \dot{M}$ & $\Delta \log \dot{M}$ & $\log D_{\text {mom }}$ & $\Delta \log D_{\mathrm{m}}$ \\
\hline HD 93129A & 3.71 & $\begin{array}{l}+0.10 \\
-0.10\end{array}$ & 22.5 & $\begin{array}{l}+3.4 \\
-3.0\end{array}$ & 0.10 & \pm 0.02 & 6.17 & $\begin{array}{l}+0.16 \\
-0.16\end{array}$ & 94.8 & $\begin{array}{l}+41.3 \\
-28.8\end{array}$ & -4.58 & $\begin{array}{l}+0.10 \\
-0.10\end{array}$ & 30.40 & $\begin{array}{l}+0.14 \\
-0.14\end{array}$ \\
\hline HD 93128 & 4.01 & $\begin{array}{l}+0.10 \\
-0.10\end{array}$ & 10.4 & $\begin{array}{l}+1.5 \\
-1.3\end{array}$ & 0.10 & \pm 0.02 & 5.66 & $\begin{array}{l}+0.13 \\
-0.13\end{array}$ & 39.8 & $\begin{array}{l}+17.2 \\
-12.0\end{array}$ & -5.58 & $\begin{array}{l}+0.17 \\
-0.24\end{array}$ & 29.22 & $\begin{array}{l}+0.19 \\
-0.26\end{array}$ \\
\hline HD 93250 & 3.96 & +0.10 & 15.9 & $\begin{array}{l}+2.4 \\
-2.1\end{array}$ & 0.10 & \pm 0.02 & 6.01 & $\begin{array}{l}+0.13 \\
-0.13\end{array}$ & 83.3 & $\begin{array}{l}+36.0 \\
-25.1\end{array}$ & -5.46 & $\begin{array}{l}+0.11 \\
-0.16\end{array}$ & 29.45 & $\begin{array}{l}+0.14 \\
-0.18\end{array}$ \\
\hline HD 66811 & 3.59 & $\begin{array}{l}+0.09 \\
-0.09\end{array}$ & 19.4 & $\begin{array}{l}+4.3 \\
-3.5\end{array}$ & 0.20 & \pm 0.03 & 5.90 & $\begin{array}{l}+0.18 \\
-0.19\end{array}$ & 53.9 & $\begin{array}{l}+30.8 \\
-19.5\end{array}$ & -5.06 & $\begin{array}{l}+0.13 \\
-0.13\end{array}$ & 29.74 & $\begin{array}{l}+0.18 \\
-0.18\end{array}$ \\
\hline HD 303308 & 3.91 & $\begin{array}{l}-0.10 \\
-0.10\end{array}$ & 11.5 & $\begin{array}{l}+1.7 \\
-1.5\end{array}$ & 0.075 & ${ }_{-0.01}^{+0.02}$ & 5.53 & $\begin{array}{l}-0.14 \\
-0.14\end{array}$ & 39.0 & $\begin{array}{l}+16.8 \\
-11.7\end{array}$ & -5.79 & $\begin{array}{l}-0.13 \\
-0.13\end{array}$ & 29.03 & $\begin{array}{l}-1.16 \\
-0.16\end{array}$ \\
\hline HD 14947 & 3.48 & $\begin{array}{l}-0.10 \\
+0.09\end{array}$ & 16.8 & $\begin{array}{l}+2.5 \\
-2.2\end{array}$ & 0.20 & \pm 0.03 & 5.70 & $\begin{array}{l}-0.14 \\
-0.14\end{array}$ & 30.7 & $\begin{array}{l}+13.1 \\
{ }_{-9.2}\end{array}$ & -5.07 & $\begin{array}{l}-0.10 \\
-0.10\end{array}$ & 29.71 & $\begin{array}{l}-0.13 \\
-0.13\end{array}$ \\
\hline HD 15558 & 3.81 & $\begin{array}{l}+0.10 \\
+0.10\end{array}$ & 18.2 & $\begin{array}{l}+2.7 \\
-2.4\end{array}$ & 0.10 & \pm 0.02 & 5.93 & $\begin{array}{l}-0.14 \\
+0.14 \\
-0.14\end{array}$ & 78.7 & $\begin{array}{l}-9.2 \\
+33.8 \\
-23.7\end{array}$ & -5.25 & $\begin{array}{l}-0.10 \\
+0.11 \\
-0.19\end{array}$ & 29.62 & $\begin{array}{l}-0.13 \\
+0.14 \\
-0.21\end{array}$ \\
\hline HD 193682 & 3.65 & $\begin{array}{l}-0 \\
-0\end{array}$ & 13.1 & $\begin{array}{l}+2.0 \\
-1.7\end{array}$ & 0.20 & \pm 0.02 & 5.60 & $\begin{array}{l}-0 \\
+0 \\
-0\end{array}$ & 27.9 & $\begin{array}{l}-23.1 \\
+11.7\end{array}$ & -5.76 & $\begin{array}{l}-0 \\
+0 \\
-0\end{array}$ & 29.04 & $\begin{array}{l}-0.21 \\
+0.16 \\
-0.30\end{array}$ \\
\hline HD 15629 & 3.71 & & 12.8 & $\begin{array}{l}+1.9 \\
-1.7\end{array}$ & 0.08 & $\begin{array}{l}+0.02 \\
-0.01\end{array}$ & 5.60 & $\begin{array}{l}+0 \\
-0\end{array}$ & 30.4 & $\begin{array}{l}+13.1 \\
-9.1\end{array}$ & -5.89 & $\begin{array}{l}-0 \\
+0 \\
-0\end{array}$ & 28.96 & $\begin{array}{l}-0.30 \\
+0.15 \\
-0.22\end{array}$ \\
\hline HD 210839 & 3.58 & $\begin{array}{l}-0 \\
+0 \\
-0\end{array}$ & 21.1 & $\begin{array}{l}-1.1 \\
+5.5 \\
-4.4\end{array}$ & 0.10 & \pm 0.02 & 5.83 & $\begin{array}{l}-0 \\
+0 \\
-0\end{array}$ & 62.2 & $\begin{array}{l}-3.1 .5 \\
+21.9\end{array}$ & -5.16 & $\begin{array}{l}-0 \\
+0 \\
+0\end{array}$ & 29.65 & $\begin{array}{l}-0.22 \\
+0.21 \\
-0.21\end{array}$ \\
\hline HD 190864 & 3.57 & & 12.3 & $\begin{array}{l}+1.8 \\
-1.6\end{array}$ & 0.15 & \pm 0.03 & 5.41 & $\begin{array}{l}+0.13 \\
-0.13\end{array}$ & 20.3 & $\begin{array}{l}+8.7 \\
-6.1\end{array}$ & -5.86 & $\begin{array}{l}+0 \\
-0\end{array}$ & 28.88 & $\begin{array}{l}+0.18 \\
-0.20\end{array}$ \\
\hline HD 192639 & 3.47 & ${ }_{-0}^{+0}$ & 18.7 & $\begin{array}{l}-1.0 \\
+2.8 \\
-2.4\end{array}$ & 0.20 & \pm 0.03 & 5.68 & $\begin{array}{l}-0.13 \\
+0.14 \\
-0.14\end{array}$ & 37.5 & $\begin{array}{l}-6.1 \\
+16.1 \\
-112\end{array}$ & -5.20 & $\begin{array}{l}-0 . \\
+0 . \\
-0\end{array}$ & 29.57 & $\begin{array}{l}-0.20 \\
+0.14 \\
-0.14\end{array}$ \\
\hline HD 193514 & 3.32 & & 19.3 & $\begin{array}{l}+2.9 \\
-2.5\end{array}$ & 0.10 & \pm 0.02 & 5.68 & $\begin{array}{l}-0.14 \\
+0.14 \\
-0.14\end{array}$ & 28.2 & $\begin{array}{l}-11.2 \\
{ }_{-8.5}^{12.1}\end{array}$ & -5.46 & ${ }_{-0.13}^{+0.12}$ & 29.33 & $\begin{array}{l}-0.14 \\
+0.15 \\
-0.16\end{array}$ \\
\hline HD 24912 & 3.56 & $\begin{array}{l}+4 \\
-1\end{array}$ & 14.0 & $\begin{array}{l}+2.1 \\
-1.8\end{array}$ & 0.15 & \pm 0.03 & 5.42 & $\begin{array}{l}+0.13 \\
-0.13\end{array}$ & 26.1 & $\begin{array}{l}+10.9 \\
-7.6\end{array}$ & -5.97 & $\begin{array}{l}+0.12 \\
-0.19\end{array}$ & 28.80 & $\begin{array}{l}+0.15 \\
-0.21\end{array}$ \\
\hline HD 203064 & 3.60 & $\begin{array}{l}-4 \\
+4 \\
-4\end{array}$ & 15.7 & $\begin{array}{l}-1.0 \\
+2.3 \\
-2.0\end{array}$ & 0.10 & \pm 0.02 & 5.50 & $\begin{array}{l}-0 \\
+0 \\
-0\end{array}$ & 35.9 & $\begin{array}{l}-1.6 \\
{ }_{-10.9}\end{array}$ & -5.85 & ${ }_{-0}^{+0}$ & 28.95 & $\begin{array}{l}-0.21 \\
+0.16 \\
-0.22\end{array}$ \\
\hline HD 217086 & 3.72 & & 8.6 & $\begin{array}{l}+1.3 \\
-1.1\end{array}$ & 0.15 & \pm 0.03 & 5.05 & $\begin{array}{l}-0 \\
-0\end{array}$ & 14.2 & $\begin{array}{l}+6.3 \\
+4.0\end{array}$ & $\leq-6.64$ & $\begin{array}{l}+0 \\
+0\end{array}$ & $\leq 28.03$ & $\begin{array}{l}+0.21 \\
-0.69\end{array}$ \\
\hline HD 13268 & 3.48 & $\begin{array}{l}+0.11 \\
-0.08\end{array}$ & 10.3 & $\begin{array}{l}-1.1 \\
+1.5 \\
-1.3\end{array}$ & 0.25 & \pm 0.03 & 5.05 & $\begin{array}{l}+0 \\
-0\end{array}$ & 11.7 & $\begin{array}{l}-4.0 \\
+5.2 \\
-3.3\end{array}$ & $\leq-6.59$ & $\begin{array}{l}{ }_{-0.34}^{+0.16} \\
-n_{0}\end{array}$ & $\leq 28.05$ & $\begin{array}{l}-0.09 \\
+0.18 \\
-0.36\end{array}$ \\
\hline HD 210809 & 3.12 & $\begin{array}{l}-0.10 \\
-0.10\end{array}$ & 21.2 & $\begin{array}{l}+3.3 \\
+2.8\end{array}$ & 0.14 & \pm 0.03 & 5.60 & $\begin{array}{l}-0.18 \\
-0.19\end{array}$ & 21.7 & $\begin{array}{l}+9.4 \\
-6.6\end{array}$ & -5.28 & $\begin{array}{l}-0.12 \\
-0.12\end{array}$ & 29.51 & $\begin{array}{l}+0.15 \\
-0.15\end{array}$ \\
\hline HD 207198 & 3.46 & $\begin{array}{l}-0 \\
+0 \\
-0\end{array}$ & 16.6 & $\begin{array}{l}-2.0 \\
+2.5 \\
-2.2\end{array}$ & 0.12 & \pm 0.03 & 5.47 & $\begin{array}{l}-0.19 \\
+0.13 \\
-0.13\end{array}$ & 29.0 & $\begin{array}{l}-6.0 \\
{ }_{-8.5}^{+12.5}\end{array}$ & -5.75 & $\begin{array}{l}-0.12 \\
+0.15 \\
-0.17\end{array}$ & 28.99 & $\begin{array}{l}-0.15 \\
+0.17 \\
-0.19\end{array}$ \\
\hline HD 30614 & 2.99 & & 32.5 & $\begin{array}{l}+4.8 \\
-4.2\end{array}$ & 0.10 & \pm 0.02 & 5.83 & $\begin{array}{l}-0.13 \\
-0.14\end{array}$ & 37.6 & $\begin{array}{l}-6.1 \\
+16.1 \\
-11.2\end{array}$ & -5.22 & $\begin{array}{l}-0.17 \\
+0.10 \\
-0.10\end{array}$ & 29.53 & $\begin{array}{l}-0.19 \\
+0.14 \\
-0.14\end{array}$ \\
\hline HD 209975 & 3.22 & & 22.9 & $\begin{array}{l}-4.2 \\
+3.4 \\
-3.0\end{array}$ & 0.10 & \pm 0.02 & 5.69 & $\begin{array}{l}-0 \\
+0 \\
-0\end{array}$ & 31.4 & $\begin{array}{l}-11.2 \\
+13.4\end{array}$ & -5.67 & $\begin{array}{l}x_{-1}^{+6} \\
-1\end{array}$ & 29.12 & $\begin{array}{l}-0.14 \\
+0.13 \\
-0.19\end{array}$ \\
\hline HD 18409 & 3.04 & + & 16.3 & $\begin{array}{l}+2.5 \\
-2.1\end{array}$ & 0.14 & \pm 0.03 & 5.29 & $\begin{array}{l}+0 \\
-0\end{array}$ & 10.6 & $\begin{array}{l}+4.4 \\
-3.1\end{array}$ & -5.99 & $\begin{array}{l}+0.20 \\
-0.17\end{array}$ & 28.66 & $\begin{array}{l}+0.22 \\
-0.19\end{array}$ \\
\hline HD 191423 & 3.60 & $\begin{array}{l}-0 \\
+0 \\
-0\end{array}$ & 12.9 & $\begin{array}{l}-2.1 \\
+1.9 \\
-1 .\end{array}$ & 0.20 & \pm 0.03 & 5.23 & $\begin{array}{l}-0 \\
+0 \\
-0\end{array}$ & 24.6 & $\begin{array}{l}-5.1 \\
+11.2 \\
-7.0\end{array}$ & $\leq-6.39$ & $\begin{array}{l}-0 \\
+0 \\
-0\end{array}$ & $\leq 28.03$ & $\begin{array}{l}-0.19 \\
+0.14 \\
-0.22\end{array}$ \\
\hline HD 149757 & 3.85 & $\begin{array}{l}{ }_{-0.08}^{+0.10} \\
-0.08\end{array}$ & 8.9 & $\begin{array}{l}-1.1 \\
+1.3 \\
-1.1\end{array}$ & 0.17 & \pm 0.03 & 4.87 & $\begin{array}{l}{ }_{-0.13}^{+0.13} \\
-0.13\end{array}$ & 20.2 & $\begin{array}{l}+8.8 \\
+8.7\end{array}$ & $\leq-6.75$ & $\begin{array}{l}+0.17 \\
-0.66\end{array}$ & $\leq 27.72$ & $\begin{array}{l}+0.19 \\
-0.66\end{array}$ \\
\hline
\end{tabular}

our final model), we added this additional uncertainty in our model (i.e., $1500 \mathrm{~K}$ ) to the usual error of $1000 \mathrm{~K}$.

Gravities. The errors in the derived $\log g$-values, $\Delta \log g$, were consistently taken to be \pm 0.10 due to the rather good fit quality of the Balmer line wings. It has to be noted, however, that these values are "only" effective values, contaminated by the centrifugal forces present due to rotation. In order to obtain the "true" gravities needed to calculate the masses, one has to apply a "centrifugal correction". This has previously been done by Herrero et al. (1992) and Vacca et al. (1996), who argued that the centrifugal acceleration averaged over the stellar disk can be approximated by the projected centrifugal velocity,

$\left\langle g_{\text {cent }}\right\rangle=\frac{\left\langle\left(V_{\text {rot }} \sin \theta\right)^{2}\right\rangle}{R_{\star}} \approx \frac{\left(V_{\text {rot }} \sin i\right)^{2}}{R_{\star}}$,

where $\theta$ is the stellar co-latitude. However, in neither of these publications this expression has been actually derived, and we could not find such a derivation anywhere. Since such a derivation is inevitable, especially with respect to a thorough error analysis, we decided to calculate the desired centrifugal correction from first principles. In Appendix A, we have outlined the solution of the problem, and it turned out (neglecting any distortion of the stellar radius) that Eq. (3) is correct, and that the underlying errors (originating mostly from statistical arguments) can be summarized by

$\frac{\Delta\left\langle g_{\text {cent }}\right\rangle^{2}}{\left\langle g_{\text {cent }}\right\rangle^{2}} \approx\left(\frac{\Delta R_{\star}}{R_{\star}}\right)^{2}+\left(\frac{\Delta f}{f}\right)^{2}$, with
$\frac{\Delta f}{f} \approx \frac{3}{4} \frac{1}{ \pm \sqrt{5}-1} \approx{ }_{-0.23}^{+0.61}$,

assuming that the projected rotational velocities can be measured with high precision. Since

$g_{\text {true }}=g+\left\langle g_{\text {cent }}\right\rangle$

the total error of the "true" gravity follows from

$\left(\Delta \log g_{\text {true }}\right)^{2} \approx \frac{(g \Delta \log g)^{2}+\left(\left\langle g_{\text {cent }}\right\rangle \Delta \log \left\langle g_{\text {cent }}\right\rangle\right)^{2}}{g_{\text {true }}^{2}}$,

with

$\Delta \log \left\langle g_{\text {cent }}\right\rangle=\log \left(1+\frac{\Delta\left\langle g_{\text {cent }}\right\rangle}{\left\langle g_{\text {cent }}\right\rangle}\right) \approx \frac{\Delta\left\langle g_{\text {cent }}\right\rangle}{\left\langle g_{\text {cent }}\right\rangle} \log e$.

Remarkably, the error in $\log g_{\text {true }}$ is of the same order as the adopted error for $\log g$, i.e., 0.1 (and sometimes even smaller), since the true gravity can become considerably larger than the effective value due to the centrifugal correction, whereas the error with respect to this correction remains rather low. 
Helium abundance. The model calculations of both HD 303308 and HD 15629 show an under-abundance in helium of $Y_{\mathrm{He}}=0.075$ and $Y_{\mathrm{He}}=0.08$, respectively. Due to the good fit quality of the He I and He II lines (see Figs. 8 and 7) and the low values found for $Y_{\mathrm{He}}$, only small variations are possible. We, therefore, estimate an error of not more than $\Delta Y_{\mathrm{He}}=+0.02 /-0.01$. In the case of HD 303308 the formal under-abundance might be easily explained by its binarity, i.e., by a possible contamination from the companion, but in the case of HD 15629 the situation is different. For this star we found the same value as determined by Herrero et al. (1992), although we have used a completely different code and accounted for line-blocking/blanketing. Since it would be very difficult to justify such an under-abundance in physical terms, we refrain from any explanation and will keep this star in mind as an objective for further investigations.

For stars with "normal" helium abundance (i.e., $\left.Y_{\mathrm{He}}=0.10\right)$, the fit quality is good and suggests an error of $\Delta Y_{\mathrm{He}}= \pm 0.02$.

For objects with slightly increased values in $Y_{\mathrm{He}}$ (i.e., $Y_{\mathrm{He}}=0.12$ to 0.15 ), we deduced an error in helium abundance of $\Delta Y_{\mathrm{He}}= \pm 0.03$ which is consistent with the values given by Herrero et al. (2002). The last "group" of stars are those for which we found a definite over-abundance in helium, i.e., $Y_{\mathrm{He}}=0.20$ to 0.25 . The error estimate is the same as before, namely $\Delta Y_{\mathrm{He}}= \pm 0.03$. Even for HD 13268 with the highest abundance found throughout our analysis $\left(Y_{\mathrm{He}}=0.25\right)$, we estimate an error of the same order, since the fit quality is extremely good.

Radii. As is well known, one of the largest sources of error concerning any derived parameter (mass, luminosity and massloss rate, see below) for Galactic objects comprises the uncertainty in the stellar radius due to uncertain distances or absolute visual magnitudes $M_{\mathrm{v}}$, respectively. For a thorough discussion we refer the reader to Markova et al. (2003); in the present investigation we adopt a general uncertainty of $\Delta M_{\mathrm{v}}= \pm 0.3$ as a representative value for all our objects. Although the individual $1-\sigma$ values are different and smaller in most cases, we adopt this value in view of the different sources from which our data is derived. The only exceptions are $\zeta$ Pup and $\lambda$ Cep, for which larger uncertainties $( \pm 0.43$ and $\pm 0.5 \mathrm{mag})$ are adopted, as explained in Sect 4.

Since we calculate the stellar radius from both $M_{\mathrm{v}}$ and theoretical model fluxes (Eq. (1)) and since $H_{\lambda} \approx B_{\lambda}\left(T_{\text {rad }}\right) \propto T_{\text {eff }}$ in the $V$-band (Sect. 4 ), the corresponding error is given by

$$
\begin{aligned}
& \Delta \log R_{\star} \approx 0.2 \sqrt{\left(\Delta M_{\mathrm{V}}\right)^{2}+\left(2.5 \Delta \log T_{\mathrm{eff}}\right)^{2}}, \\
& \Delta \log T_{\mathrm{eff}}=\log \left(1+\frac{\Delta T_{\mathrm{eff}}}{T_{\mathrm{eff}}}\right) .
\end{aligned}
$$

With the above estimates for $\Delta M_{\mathrm{v}}$ and $\Delta T_{\text {eff }}$, the error in the stellar radius is dominated by the uncertainty in $M_{\mathrm{v}}$ and is of the order of $\Delta \log R_{\star} \approx \pm 0.06$, i.e., roughly $15 \%$.

\subsection{Wind parameters (cf. Tables 2 and 3)}

All terminal velocities, $v_{\infty}$, which have been taken from Paper I, were found to be subjected to an uncertainty of approximately $10 \%$ as shown by Haser (1995). Here and in the following, we will neglect this uncertainty with respect to its influence on the derived mass-loss rate.

In order to address the errors in the wind-parameters $\dot{M}$ and $\beta$ (which are intimately coupled), we first have to consider the fact that any line-fit to $\mathrm{H}_{\alpha}$ does not allow to specify $\dot{M}$ itself, but only the quantity $Q$, as extensively discussed in Paper I,

$Q=\frac{\dot{M}}{R_{\star}^{1.5}}$.

The logarithmic error of this quantity can be calculated from the uncertainty in $\dot{M}$ at a given value for $R_{*}$, i.e.,

$\Delta \log Q=\log \left(1+\frac{\Delta \dot{M}}{\dot{M}}\right)_{R_{\star}=\text { const. }}$.

Remember that any change of $R_{*}$ leads to an identical fit if $\dot{M}$ is adapted in such a way that $Q$ remains constant ${ }^{4}$.

Thus, before we calculate the total error in mass-loss rate which depends on both the error in $Q$ and in $R_{*}$ via

$\Delta \log \dot{M}=\sqrt{(\Delta \log Q)^{2}+\left(\frac{3}{2} \Delta \log R_{\star}\right)^{2}}$,

we have to consider the errors in $Q$ alone. For this purpose, we distinguish between two cases:

- Stars with $H_{\alpha}$ in emission (cf. Table 2). $\dot{M}$ and $\beta$ are fit parameters and determined from the $\mathrm{H}_{\alpha}$ profile itself (for specified $R_{*}$ ). The influence of $\beta$ on the profile shape (specifically on the central emission peak and also on the part where the wings start to form) is so large that it can be determined in parallel with the mass-loss rate. In this case, we adopt $\Delta \beta= \pm 0.1$; the corresponding error in the massloss rate, which directly translates into the corresponding error in the $Q$-value, $\Delta \log Q_{1}$, has been estimated from the requirement that within these errors the fit quality of $\mathrm{H}_{\alpha}$ should remain reasonable. Note, however, that the uncertainty in mass-loss rate ( $Q$-value) depends also on the error in effective temperature, $\Delta T_{\text {eff }}$, because of the accompanying change in the ionization/excitation equilibrium of hydrogen. The corresponding error, $\Delta \log Q_{2}$, has been estimated using Eqs. (48), (49) in Paper I which covers this effect ${ }^{5}$. The total error arising from both effects (line-fit and $\left.\Delta T_{\text {eff }}\right), \Delta \log Q_{t}$, follows from the usual error propagation assuming both error sources to be independent. From the results presented in Table 2, the typical (total) error in $\log Q$ (which would also be the total error in $\log \dot{M}$ if the radius were known to high precision) is of the order of 0.05 dex, i.e., $12 \%$. Only for HD 210809, the error is of the order of $20 \%$ mainly because of the larger uncertainty in $T_{\text {eff }}$ (see above).

\footnotetext{
${ }^{4}$ Except for objects which lie close to the Eddington-limit, where the actual value of $R_{*}$ has a direct impact on the photospheric structure.

${ }^{5}$ In order to account for the effects of line-blanketing incorporated in the present work, we have used $T_{\text {rad }} \approx 0.9 T_{\text {eff }}$, cf. Sect. 7.2.
} 
- Stars with $H_{\alpha}$ in absorption (cf. Table 3). For stars with absorption profiles a different approach has to be applied since $\beta$ can no longer be derived from the profile shape (with exception of a few cases which we have commented on in the previous section). Instead, it has to be adopted from theoretical considerations, and we used $\beta=0.8$ as discussed in Sect. 4. Note that the derived mass-loss rate (actually the derived $Q$-value) is valid only for this specific value and that the dependence of $Q$ on $\beta$ is much stronger for absorption than for emission type profiles (cf. Fig. 15 in paper I). Thus, in order to obtain reliable error estimates for $Q$, we varied $\beta$ within reasonable limits and deduced, for a specified value of $R_{*}$, the corresponding upper and lower boundaries of the mass-loss rate from the fit to the observed profiles.

Generally, $\dot{M}$ will become smaller if $\beta$ is increased and vice versa. In particular, we have varied $\beta$ typically by $(+0.2 /-0.1)$ to obtain i) a conservative lower limit for $\dot{M}$ and ii) to exclude $\beta$ values below 0.7 (which are difficult to justify theoretically). Only in those case where we were able to constrain $\beta$ due to additional arguments (cf. Sect. 5), the "allowed range" of $\beta$ could be (moderately) reduced. The specific values chosen for $\beta_{\min }$ and $\beta_{\max }$ as well as the errors in $\dot{M}$ estimated in such a way are listed in Table 3 . Together with the small influence of $\Delta T_{\text {eff }}$, we obtain typical uncertainties in $\Delta \log Q_{t}$ between 0.1 to 0.2 dex, i.e., of the order of $25 . .60 \%$, which indicates the lower quantity of the derived mass-loss rates if $\mathrm{H}_{\alpha}$ is in absorption (cf. Paper I and Kudritzki \& Puls 2000).

For stars with extremely low mass-loss rates, where only an upper limit of $\dot{M}$ could be deduced (HD 217086, HD 13268, HD 191423 and HD 149757), the same procedure has been applied, such that the derived limiting values, $\dot{M}^{+}$and $\dot{M}^{-}$, are also only upper limits. Note the extreme uncertainty in $\dot{M}$ for HD 217086 and HD 149757.

\subsection{Derived quantities (cf. Table 4)}

So far, we have considered the errors for the quantities which can actually be "measured" from a spectroscopic analysis, i.e., $T_{\text {eff }}, \log g_{\text {eff }}, Y_{\mathrm{He}}, Q$ and, to a lesser extent, $\log g_{\text {true }}, \beta$, and $R_{*}$. In the following, we briefly summarize the errors in the derived quantities which are needed for our further interpretation in order to assess the achieved accuracy. All values are presented in Table 4.

At first, the error in luminosity is given by

$$
\Delta \log L \approx \sqrt{\left(4 \Delta \log T_{\mathrm{eff}}\right)^{2}+\left(2 \Delta \log R_{\star}\right)^{2}}
$$

and results in $\Delta \log L \approx \pm 0.14$, i.e., the influence of the error in $R_{*}$ is somewhat larger than that in $T_{\text {eff. }}$ The error in mass,

$$
\Delta \log M \approx \sqrt{\left(\Delta \log g_{\text {true }}\right)^{2}+\left(2 \Delta \log R_{\star}\right)^{2}}
$$

is rather large and suffers, again, from the uncertainty in radius. The error in $\dot{M}$ is found from the errors in $\log Q$ and in $\log R_{\star}$
(Eq. (11)). Finally, the modified wind-momentum rate, $D_{\text {mom }}$, is given by

$D_{\mathrm{mom}}=\dot{M} v_{\infty}\left(\frac{R_{\star}}{R_{\odot}}\right)^{0.5}=Q v_{\infty}\left(\frac{R_{\star}}{R_{\odot}}\right)^{2}$,

where the corresponding error has to be calculated from the second equality, since $Q$ (and not $\dot{M}$ ) is the actual fit quantity. The typical errors in $\log D_{\text {mom }}$ are of the same order as the errors in $\log L$ which will result in a more or less quadratic error box when plotting the wind-momentum luminosity relation.

\section{Discussion}

\subsection{The effective temperature scale for Galactic O-stars}

Our analysis was carried out using a large sample of spectral subtypes ranging from $\mathrm{O} 2$ to $\mathrm{O} 9.5$ enabling us to obtain a temperature scale for $\mathrm{O}$ supergiants, giants, and dwarfs. Figure 9 displays our current calibration of $T_{\text {eff }}$ vs. spectral type for Galactic O-type stars. From this plot, we conclude that the influence of line-blanketing redefines this temperature scale significantly. Supergiants of spectral type O2 to O9.5 are now located between roughly $43000 \mathrm{~K}$ and $30000 \mathrm{~K}$ (if we assume that the effective temperature of HD 93129A is not too wrong), whereas dwarfs of spectral type $\mathrm{O} 3$ to $\mathrm{O} 9$ are located between $47000 \mathrm{~K}$ and $32000 \mathrm{~K}$.

Our results indicate a somewhat larger influence of lineblocking on the effective temperature of dwarfs than found by Martins et al. (2002) in a comparable investigation utilizing model grids. Typically, our temperatures are lower by 1000 to $2000 \mathrm{~K}$. One has to note, however, that a significant number of our objects are fast rotators, which might be affected by gravity darkening (e.g., Cranmer \& Owocki 1995; Petrenz $\&$ Puls 1996) and hence appear cooler than their non-rotating counterparts.

Moving from dwarfs to supergiants (the temperatures of giants lie in between), we can see that our temperature scale is somewhat hotter than the scale derived by Crowther et al. (2002, line-blanketed models using CMFGEN). The differences are marginal at spectral type $\mathrm{O} 4$ but increase towards later types, where the discrepancy is of the order of $4000 \mathrm{~K}$. It should be mentioned though that the accomplished analysis and results obtained by Crowther et al. (2002) comprised extreme Magellanic Clouds objects, whereas in our sample such extreme objects are rare. Thus, it can be speculated that the derived effective temperatures are lower just because of the extreme wind-density of the objects analyzed (see below). Note also that the lower entry at $\mathrm{O} 4$ corresponds to $\zeta$ Pup. For this star (which has a much more typical wind-density), the results of both analyses (ours and the one performed by Crowther et al.) agree perfectly, with a derived value for $T_{\text {eff }}=39000 \mathrm{~K}$.

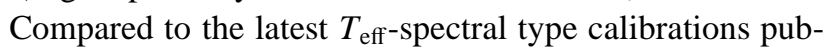
lished by Vacca et al. (1996), which is based on plane-parallel, pure $\mathrm{H} / \mathrm{He}$ model atmospheres, the differences are of the order of $4000 \mathrm{~K}$ to $8000 \mathrm{~K}$ at earliest spectral types and become minor around B0, as also shown in Fig. 9. In the following, 


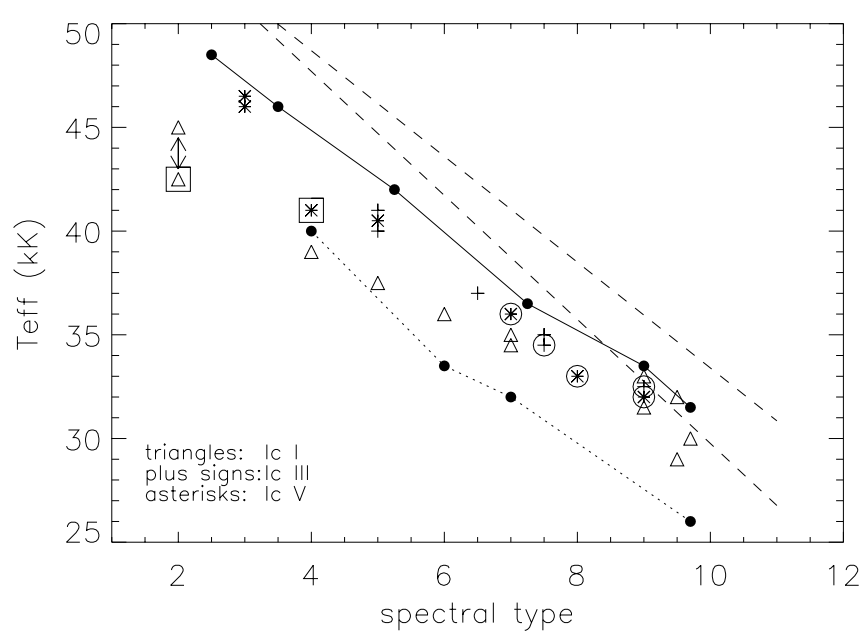

Fig. 9. $T_{\text {eff }}$ vs. spectral type for Galactic O-stars (line-blanketed models, this analysis), compared to similar investigations and results from unblanketed models. The dashed lines denote the results obtained by Vacca et al. (1996,plane-parallel, unblanketed models), where the upper and lower relation correspond to dwarfs and supergiants, respectively. The results obtained by Martins et al. (2002) are displayed by the bold line and corresponds to dwarfs (using CMFGEN+ISA). The dotted line corresponds to extreme supergiants in the Magellanic Clouds (as derived by Crowther et al. 2002 using CMFGEN). The rectangles at $\mathrm{O} 2$ and $\mathrm{O} 4$ correspond to HD 93129A and HD 303308, respectively (both stars are binary systems; see Nelan et al. 2003), whereas the arrow indicates upper and lower limits of $T_{\text {eff }}$ for HD 93129A. Circles enclose extremely fast rotators with $V_{\mathrm{r}} \sin i \geq$ $300 \mathrm{~km} \mathrm{~s}^{-1}$.

we will discuss the origin of these differences in considerable detail.

\subsection{Why lower $T_{\text {eff }}$ with blanketed models?}

As mentioned above, the inclusion of line-blanketing effects reduces the effective temperature scale significantly, when compared to the results from pure $\mathrm{H} / \mathrm{He}$ models without winds (and, to a lesser extent, when compared to the results from pure $\mathrm{H} / \mathrm{He}$ models with winds, cf. Herrero et al. 2002). As we will see in the next section, the gravities become smaller as well, at least in the typical case. On the other hand, the values for $R_{*}$ and $\dot{M}$ remain roughly at their "old" values, so that we can anticipate a significantly modified wind-momentum luminosity relation, due to the decrease in luminosity. Thus, we find severe effects concerning all problems related to $T_{\text {eff }}$ as function of spectral type (and luminosity class, due to the additional impact of mass-loss), and in the following we will investigate the question why the stars "become cooler" in more detail.

A simple answer to this question has been given in a variety of publications dealing with line-blocking/blanketing (cf. Sect. 1), and we will briefly summarize the major aspects.

Due to the presence of the multitude of metal-lines in the EUV, the flux is depressed ("blocked") in this regime, compared to a metal-line-free model. Since the total flux, however, has to be conserved the flux blocked by the lines will emerge at other frequencies. This is the case in regions where only a

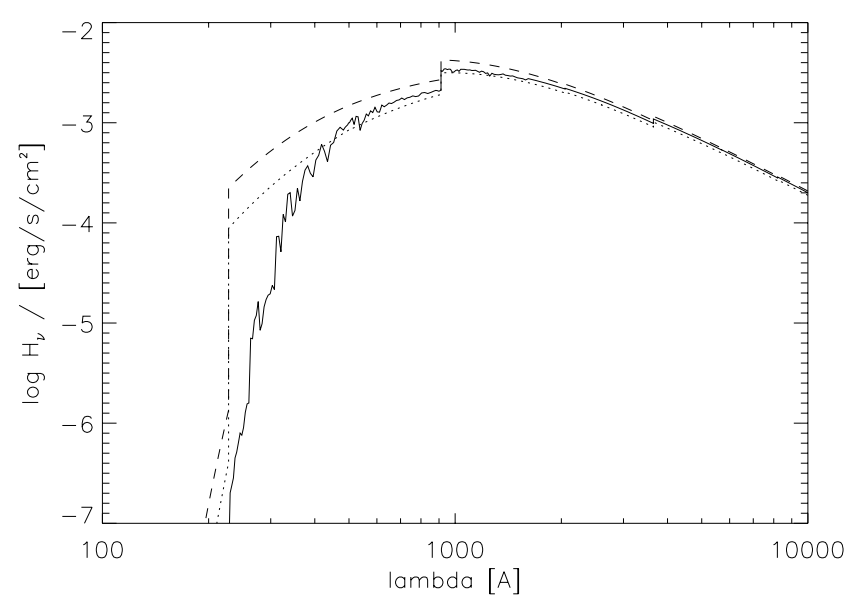

Fig. 10. Emergent Eddington flux $H_{v}$ as function of wavelength. Solid line: Current model of HD 15629 (O5V((f)) with parameters from Table 1 ( $T_{\text {eff }}=40500 \mathrm{~K}, \log g=3.7$, "model 1"). Dotted: Pure $\mathrm{H} / \mathrm{He}$ model without line-blocking/blanketing and negligible wind, at same $T_{\text {eff }}$ and $\log g$ ("model 2"). Dashed: Pure H/He model, but with $T_{\text {eff }}=45000 \mathrm{~K}$ and $\log g=3.9$ ("model 3").

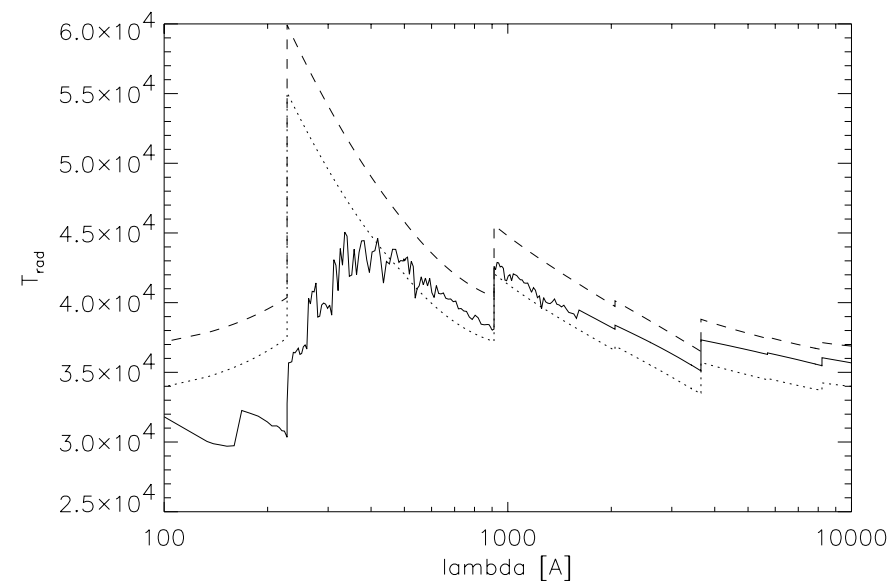

Fig. 11. As Fig. 10, but for corresponding radiation temperatures $T_{\text {rad }}$. The radiation temperature of the blanketed model 1 (solid lines) in the $V$-band and close to $\mathrm{H}_{\alpha}$ is roughly $0.9 T_{\text {eff }}$.

few lines are present, i.e., at longer wavelengths, resulting in an increase of the optical flux.

This can readily be seen in Figs. 10 and 11, where we compare the results from a prototypical example (our current model of HD $15629\left(\mathrm{O} 5 \mathrm{~V}((\mathrm{f})), T_{\text {eff }}=40500 \mathrm{~K}, \log g=3.7\right.$, hereafter "model 1") with those from a pure $\mathrm{H} / \mathrm{He}$ model (with negligible wind) at the same effective temperature and gravity ("model 2"). Note in particular that the radiation temperature in the $V$-band (and close to $\mathrm{H}_{\alpha}$ ) is given by $T_{\text {rad }} \approx 0.9 T_{\text {eff }^{6}}$, compared to the values of $0.75 \ldots 0.8 T_{\text {eff }}$ for pure $\mathrm{H} / \mathrm{He}$ models (Paper I). Thus, the ratio of the emergent fluxes longwards and shortwards from the flux maximum increases due to lineblocking/blanketing.

The process responsible for achieving this flux increase at longer wavelengths is line-blanketing. Due to the blanket of metal-lines above the continuum-forming layer, a significant fraction of photons is scattered back (or emitted in the

\footnotetext{
6 This result roughly holds for all spectral types considered here.
} 
backwards direction), such that the number density of photons ( $\propto$ mean intensity $J_{v}$ ) below this blanket is larger compared to the line-free case. These photons are (partially) thermalized, and the (electron-) temperature (around $10^{-2} \lesssim \tau_{\text {Ross }} \lesssim 2$ ) increases. Since the emergent flux is proportional to the sourcefunction at $\tau_{v}=2 / 3$ (Eddington-Barbier), and since the NLTEdeparture coefficients for the excited levels of hydrogen are close to unity for hot stars (note that the optical continuum is dominated by hydrogen bf-processes), an increase in temperature directly translates into an increase of the optical flux.

Thus, if we determined effective temperatures from optical continuum fluxes (concerning the failure of such a method, see Hummer et al. 1988), the reduction of $T_{\text {eff }}$ would be easily explained: Line-blanketed models have optical fluxes similar to those from unblanketed models at higher $T_{\mathrm{eff}}$.

Although the actual analysis of $T_{\text {eff }}$ for hot stars depends on the helium ionization equilibrium (see below), the above finding allows us to understand why the derived stellar radii remain almost unaltered: Since we "measure" these radii from a comparison of $M_{\mathrm{v}}$ (which of course is independent of the model) with theoretical model fluxes in the $V$-band (cf. Eq. (1)), where the latter depend almost linearly on the corresponding $T_{\text {rad }}$ (Rayleigh-Jeans regime), the ratio between "old" and new radii can be approximated by

$\frac{R_{\star}^{\text {new }}}{R_{\star}^{\text {old }}} \approx \frac{0.9 T_{\mathrm{eff}}^{\text {new }}}{0.8 T_{\mathrm{eff}}^{\text {old }}}$

and is close to unity in any case, since $T_{\mathrm{eff}}^{\text {new }}<T_{\mathrm{eff}}^{\mathrm{old}}$.

A closer inspection. As just pointed out, the actual determination of $T_{\text {eff }}$ for hot stars exploits the sensitivity of the He I/He II ionization equilibrium on temperature. Figure 12 shows the corresponding ionization fractions for model 1 and 2 (compare with Fig. 3 in Herrero et al. 2002), as well as the results for a hotter, pure $\mathrm{H} / \mathrm{He}$ model (again with negligible wind) at $T_{\text {eff }}=45000 \mathrm{~K}$ and $\log g=3.9$ (dashed curve, "model 3"). In the formation region of photospheric lines $\left(\tau_{\text {Ross }} \lesssim 5 \times 10^{-2}\right.$, onset of wind at lower values), the ionization fractions of both He I and He II are similar for model 1 and 3; in contrast, model 2 produces significantly more $\mathrm{He}$ I and He II:

Line-blanketed models of hot stars have photospheric He ionization fractions similar to those from unblanketed models at higher $T_{\text {eff }}$ (and higher $\log g$, see below).

The final question then is: What determines the displayed behaviour of the ionization fractions? If we concentrated in Fig. 11, this behaviour would remain unclear. In model 1, the emergent flux shortwards of the He II-Lyman-edge is lowest. In so far, we would erroneously conclude that this model has the highest population of He II (at least, regarding the groundstate), in contrast to what is displayed in Fig. $12^{7}$. We have

\footnotetext{
7 The reason that model 1 has the lowest emergent flux is given by the fact that for this model the He II continuum becomes optically thick already in the wind, since the He II population is larger there, compared to the other models.
}

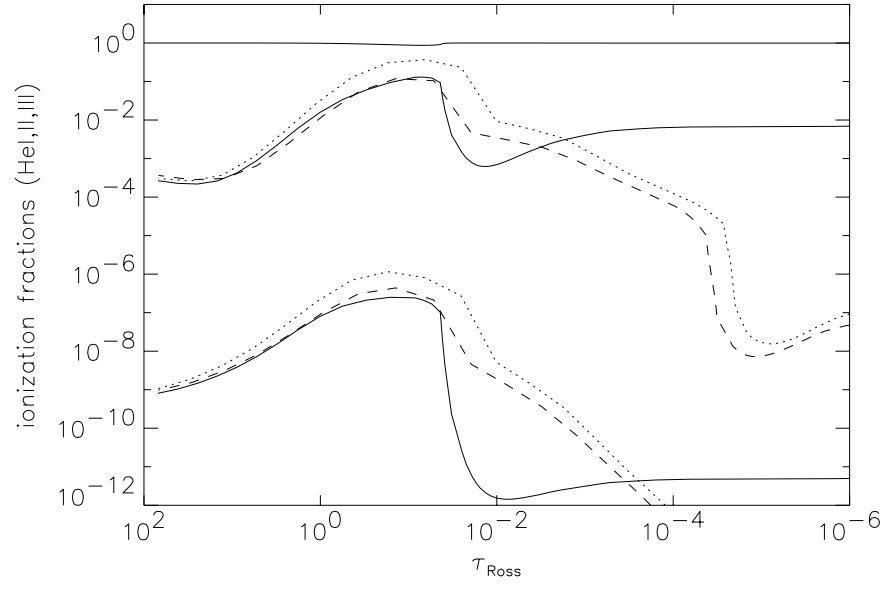

Fig. 12. Ionization fractions of He for the different models from Fig. 10, as function of $\tau_{\text {Ross. }}$. From top to bottom: He III (blanketed model only), He II and He I, respectively. Note that the fractions for our current blanketed model (solid) coincide with the fractions for the hotter, unblanketed one (dashed).

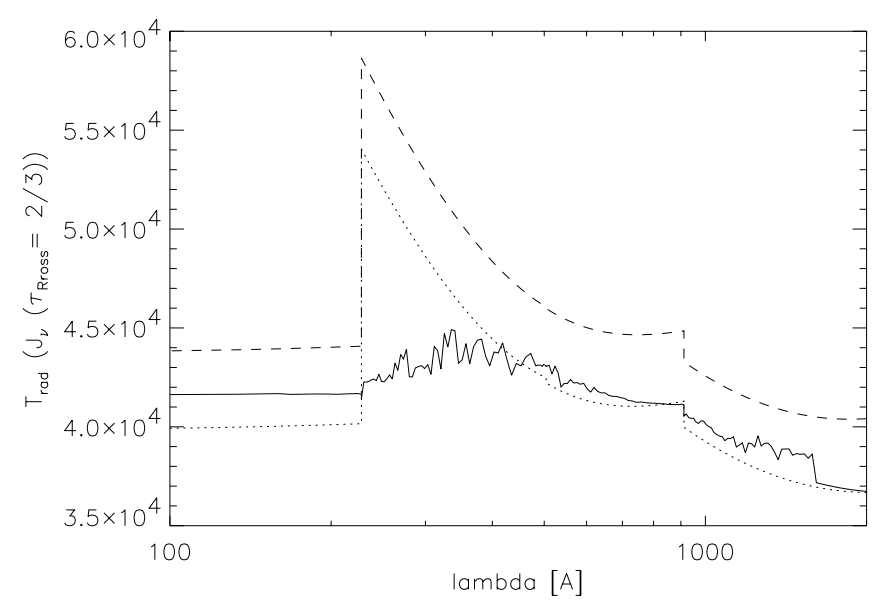

Fig. 13. As Fig. 11, but with radiation temperatures calculated from mean intensity $J_{v}$ at $\tau_{\text {Ross }}=2 / 3$. Note the difference in the He II Lyman continuum $(\lambda<229 \AA)$. Whereas the Lyman-flux is lowest for the blanketed model (Fig. 11), the corresponding mean intensities lie in between the results of both unblanketed $\mathrm{H} / \mathrm{He}$ models.

to remember, however, that the ionization equilibrium is controlled by the balance between ionization (dependent on the local photon-density) and recombination (dependent on the local electron temperature and density).

Thus, in order to understand the run of ionization, we have to consider the mean intensity, plotted in Fig. 13 as corresponding radiation temperature $\left(J_{v}:=B_{v}\left(T_{\text {rad }}(v)\right)\right.$ for a depth of $\tau_{\text {Ross }}=2 / 3$. Most important and in contrast to Fig. 11 (emergent flux) is the fact that the mean intensities shortwards of the He II Lyman edge are now ordered in the following sequence (from lowest to highest values): model 2, 1 and 3, i.e., the results for the blanketed model lie in between the results of the unblanketed ones. This is true not only for $\tau_{\text {Ross }}=2 / 3$, but also for the complete photosphere, and it is also true for the run of the electron temperature, lying in between the temperature stratifications for model 2 and 3 due to the effects of lineblanketing as discussed above. 
It is well known that the ionization balance (or more correctly, the ratio between the ground state occupation numbers of ion $k$ and ion $k+1$ ) can be approximated by (e.g., Abbott \& Lucy 1985; Puls et al. 2000)

$\frac{n_{1, k}}{n_{1, k+1}}=n_{\mathrm{e}} \sqrt{\frac{T_{\mathrm{rad}}}{T_{\mathrm{e}}}}\left(\frac{n_{1, k}}{n_{1, k+1} n_{\mathrm{e}}}\right)_{T_{\mathrm{rad}}}^{*}$

with $n_{\mathrm{e}}$ being the actual electron-density and $T_{\text {rad }}$ being the radiation temperature at the ionization edge. The bracket denotes the corresponding LTE-value evaluated at $T_{\mathrm{rad}}{ }^{8}$. Without those constants which are identical for a specific ion, we have

$$
\begin{aligned}
& \frac{n_{1}(\mathrm{He} \mathrm{II})}{n(\mathrm{He} \mathrm{III})} \propto \frac{n_{\mathrm{e}}}{T_{\mathrm{rad}}(229 \AA) \sqrt{T_{\mathrm{e}}}} \exp \left(\frac{1.4388 \times 10^{8}}{229 \times T_{\mathrm{rad}}(229 \AA)}\right) \\
& \frac{n_{1}(\mathrm{He} \mathrm{I})}{n(\mathrm{He} \text { III })} \propto \frac{n_{\mathrm{e}}}{T_{\mathrm{rad}}(504 \AA) \sqrt{T_{\mathrm{e}}}} \\
& \times \exp \left(\frac{1.4388 \times 10^{8}}{504 \times T_{\mathrm{rad}}(504 \AA)}\right) \frac{n_{1}(\mathrm{He} \mathrm{II})}{n(\mathrm{He} \text { III })}
\end{aligned}
$$

Using these approximations, we have convinced ourselves in Fig. 14 that the similarity of the He ionization equilibrium for model 1 and 3 as well as the larger population of He I and He II for model 2 can be explained by three facts:

i) the run of the electron temperatures, where the values for the blanketed model lie in between the ones for the unblanketed models (back-warming);

ii) the run of the radiation temperatures, which are rather similar to $T_{\mathrm{e}}$ (continua optically thick in the photosphere!), although somewhat higher ${ }^{9}$.

iii) the higher electron density $n_{\mathrm{e}}$ for model 3 , because of the higher gravity in this case $(\log g=3.9$ vs. $\log g=3.7)$. If we had compared models with identical gravities, model 3 would have yielded the highest ionization degree, which is just compensated because of the increased recombination due to the higher electron density.

In summary, the He ionization equilibrium of our blanketed model 1 and the hotter, unblanketed model 3 are similar because of backwarming (increasing $T_{\mathrm{e}}$ and, thus, $T_{\mathrm{rad}}$ at the edges in model 1) and because of the higher gravity in model 3 (increasing the photospheric recombination).

Figure 15 finally displays the corresponding profiles for He I 4471. Obviously, the results for model 1 and 3 are indistinguishable, whereas model 2 produces a much stronger profile. Thus, a spectroscopic analysis of hot stars, based on the He ionization equilibrium and performed by means of blanketed models, will usually result in parameters at lower $T_{\text {eff }}$ and lower $\log g$, compared to an analysis utilizing pure $\mathrm{H} / \mathrm{He}$ models.

The parameters derived from $\mathrm{He}$, of course, have to consistently produce the other (optical) lines from hydrogen

\footnotetext{
${ }^{8}$ Actually, this expression needs to be modified by a factor containing certain branching ratios with respect to ordinary and metastable levels, which in the following is of no concern.

${ }^{9}$ Because of the usual effect that for ground states and close to the surface, $J_{v}>B_{v}(T)$, and the additional increase of $J_{v}$ due to backscattered photons from above in case of the blanketed model 1.
}

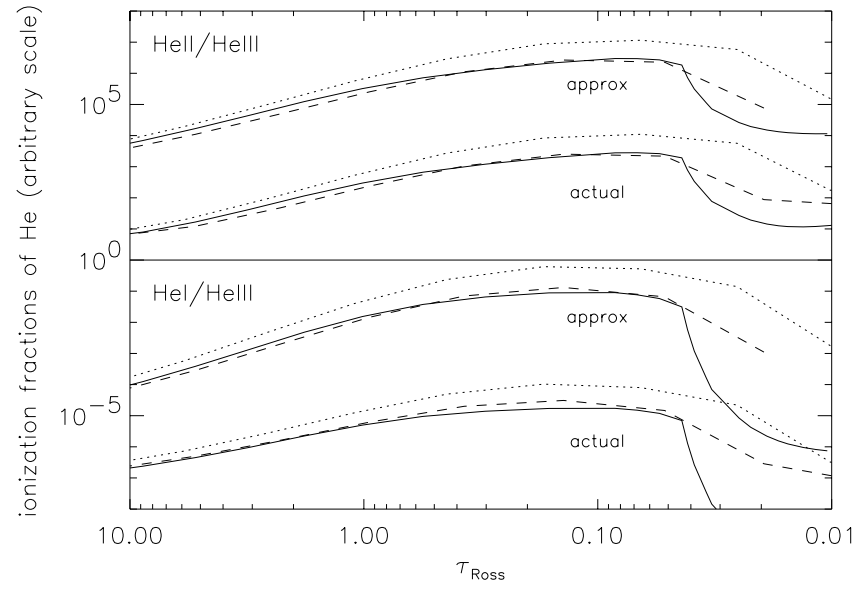

Fig. 14. As Fig. 12, but for ionization ratios He II/He III (upper panel) and $\mathrm{He} \mathrm{I} / \mathrm{He}$ III (lower panel). Both panels show the actual ratios for all three models as well as the ratios as approximated by Eq. (15), using mean intensities at the ionization edge. The offset between all four arrays of curves is arbitrary. Obviously, the approximation is a good representation for the actual situation (see text).

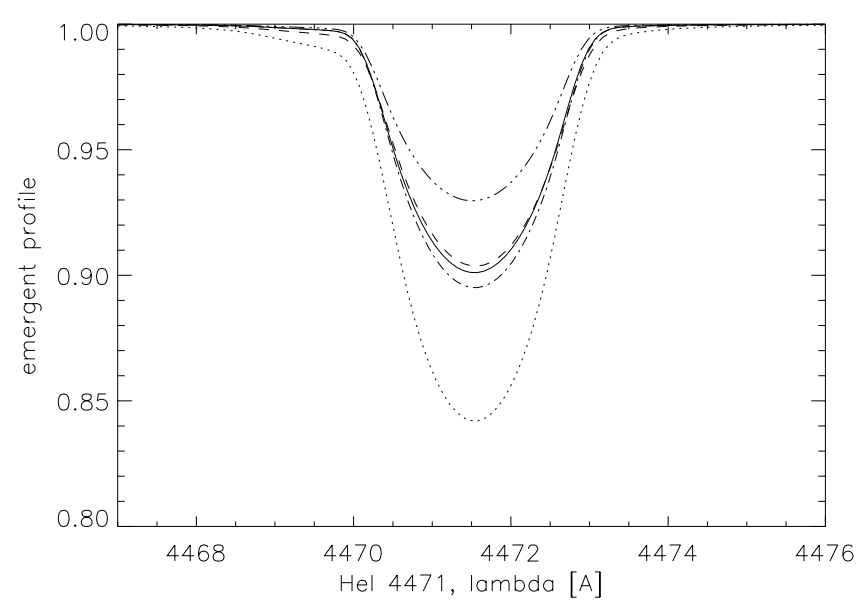

Fig. 15. He $\mathrm{I} 4471$ line $\left(V_{\mathrm{r}} \sin i=90 \mathrm{~km} \mathrm{~s}^{-1}\right)$ for all three models from Fig. 10. Whereas the profiles model 1 and model 3 coincide, model 2 produces a much stronger line (see also Fig. 12). The dashed-dotted profile results from a model similar to our current blanketed one, but with negligible mass-loss. Obviously, the presence or absence of a weak wind (model 1 has a mass-loss rate of $1.3 \times 10^{-6} M_{\odot} / \mathrm{yr}$ ) has no effect on the temperature analysis. The weakest profile shows the influence of a strong wind: the underlying model again is similar to model 1 , but with a mass-loss rate of $7.5 \times 10^{-6} M_{\odot} / \mathrm{yr}$.

and He II. Since for hotter stars the He II lines $\lambda \lambda 4200,4541$ are preferentially fed by recombination from He III (which remains the dominant ion with and without blocking), they remain almost unaffected by temperature variations and react mainly (but weakly) on gravity (cf. the corresponding sequence of He II lines in Fig. 8). On the other hand, the hydrogen Balmer lines remain fairly unaltered if temperature and gravity are changed in parallel, which needs to be done in any case if HeI is to be preserved.

It is hence possible to obtain line-fits of almost equal quality from blanketed and unblanketed models, if the former have lower $T_{\text {eff }}$ and $\log g$ than the latter. For physical reasons we 
prefer the former, implying that we have to accept a recalibration of stellar parameters as a function of spectral type.

In the following we will see that there is also another possibility: If the hydrogen lines "forbid" a decrease in gravity, we might be able to obtain a fit at lower $T_{\text {eff }}$ and equal $\log g$, but with a reduced helium abundance. This reduction then compensates for the increase of the $\mathrm{He}$ I fraction, which otherwise could be obtained only by a decrease in $\log g$. The He II lines must allow for such a reduction, which is possible in certain domains of the $\left(T_{\text {eff }}, \log g\right)$-plane.

From these results it becomes also clear why the $T_{\text {eff }}$ correction for supergiants is larger than for dwarfs. Supergiants have a stronger wind due to a larger luminosity. At first glance, one might speculate that the major effect is an increased backwarming effect due to an increased wind-albedo (cf. Hummer 1982; Voels et al. 1989): the "blanket" becomes denser because of the increase in line opacity and the velocity shift in the wind. However, in most cases this effect is minor compared to the influence of the wind itself. In a dense wind, the line cores are formed in the wind, and particularly $\mathrm{He} \mathrm{I}$ is significantly filled in by wind emission (Fig. 15). Thus, a larger correction to even lower $T_{\text {eff }}$ is required to match the observations, compared to an analysis based on wind-free models.

One last comment: Not only $T_{\text {eff }}$ and $\dot{M}$ have an influence on the effect of line-blocking, but also $\log g$, particularly for dwarfs. Since with increasing $\log g$ the photospheric density increases, the recombination rates of the metal-ions become enhanced, which results in a higher population of the lower ionization states. Less ionized metals have a more complex level structure whose more numerous lines then enhance the blocking and blanketing effect.

\subsection{The $T_{\text {eff }} v s$. $g$ diagram}

Figures 16 and 17 show the spectroscopist's view of the Hertzsprung-Russel diagram, namely $\log g$ vs. $T_{\text {eff }}$, which is independent of any uncertainty in the distance. Compared to the results from Paper I, a shift towards lower temperatures can be observed for all stars in our sample as indicated by the displacement vectors, which is in agreement with the results from above. As shown, we would also expect a (moderate) reduction of $\log g^{10}$, which is found for only 14 out of 24 sample stars. For eight stars, the gravities remain unaltered, and for two stars, HD 24912 and HD 207198 (luminosity class III and I, respectively), we actually had to increase $\log g$ in order to obtain a convincing fit.

If we consider those objects in more detail where the gravity remained at its old value (or had to be increased), it turns out that for 9 of the 12 objects we derived a lower helium abundance than found by Herrero et al. (1992). These cases, thus, comprise the alternative stated above: Instead of a reduced gravity, which in these cases is "forbidden" from the hydrogen Balmer lines, we obtained a reduced helium abundance. Therefore, the well-known helium discrepancy has

\footnotetext{
${ }^{10}$ Note that the values of $\log g$ from Paper I include an approximate correction for wind-effects.
}

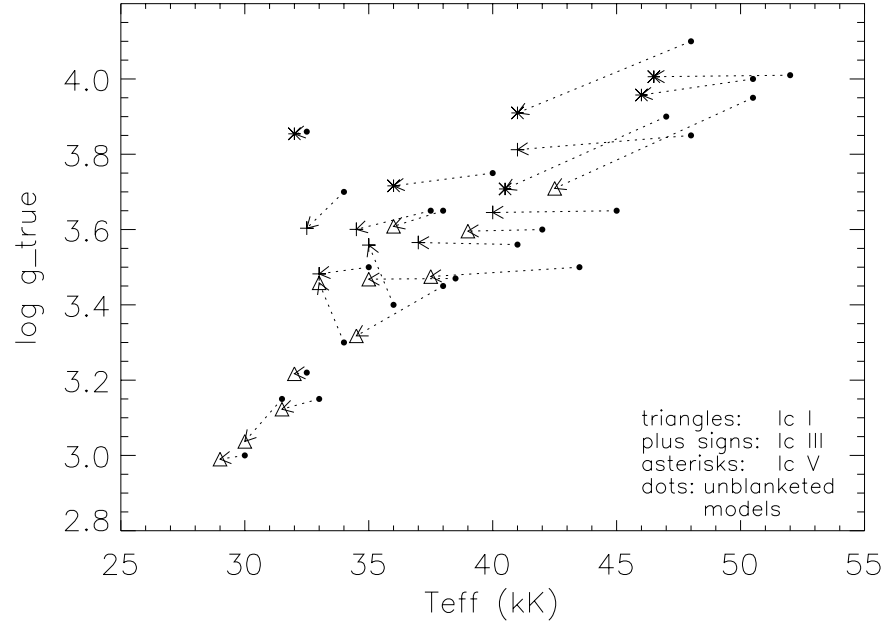

Fig. 16. True gravity $\log g_{\text {true }}$ vs. $T_{\text {eff }}$ for Galactic O-stars (this work) compared to the results from Paper I (unblanketed models, gravity corrected for wind effects and centrifugal forces). A shift towards lower temperatures can be observed for all stars in our sample, as indicated by the displacement vectors in the $T_{\text {eff- }} \log g$ plane; 14 out of 24 stars had to be shifted towards lower $\log g, 8$ objects preserved their "old" value and for two objects we had to increase the gravity (see text).

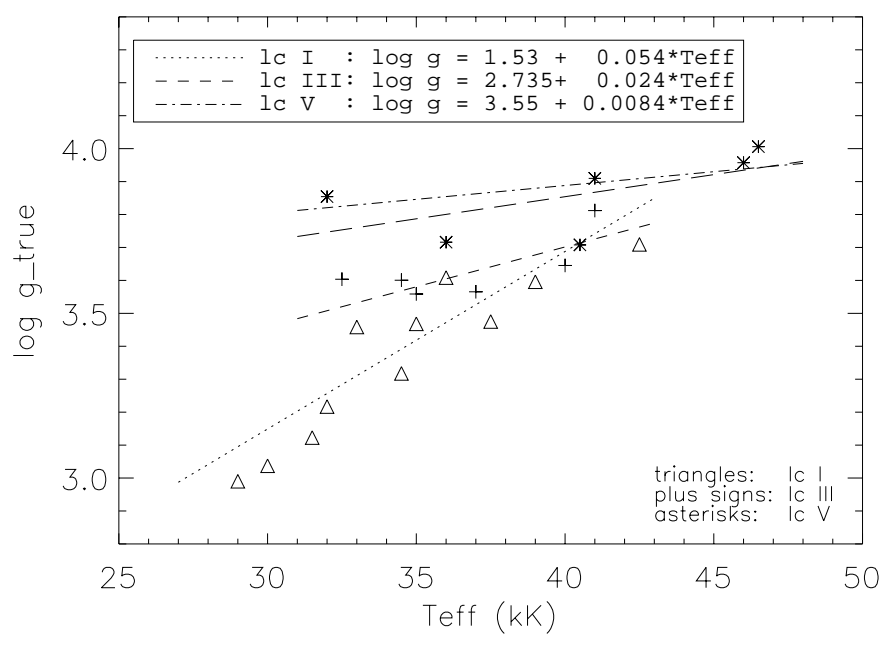

Fig. 17. True gravity $\log g_{\text {true }}$ vs. $T_{\text {eff }}$ for Galactic O-stars (this work) compared to the calibrations provided by Markova et al. (2003), evaluated in the $T_{\text {eff }}-\log g$ plane. Although almost all results agree within the typical error bars $\Delta T_{\text {eff }} \approx \pm 1500 \mathrm{~K}$ and $\Delta \log g \approx \pm 0.1$, the "outliers" HD 217086 (O7Vn, fast rotator) and HD 15629 (O5V((f))) might indicate a steeper relation for dwarfs, as indicated by the longdashed line, $\log g=3.32+0.013 T_{\text {eff }}, T_{\text {eff }}$ in $\mathrm{kK}$ (see text).

considerably been reduced by our analysis using blanketed models (see also Herrero et al. 2002).

Figure 17 again displays our new $\log g$ vs. $T_{\text {eff }}$ diagram for Galactic O-stars, but now we compare the results to a recent calibration implicitly provided by Markova et al. (2003), who have partly used the results described in the present paper. In particular, the plot serves as a consistency check, because Markova et al. derived two independent calibrations, $T_{\text {eff }}$ vs. spectral type and $\log g_{\text {true }}$ vs. spectral type. Since their calibrations are based on a linear model (with all its caveats, particularly for extreme supergiants), it is possible to combine both 
and to derive a calibration for $\log g$ vs. $T_{\text {eff. This is what we }}$ have done in Fig. 17, where also the corresponding coefficients have been tabulated as a function of luminosity class.

In the case of class I and III objects, also this new combination agrees with our results: almost all objects are within the typical error bars $\Delta T_{\mathrm{eff}} \approx \pm 1500 \mathrm{~K}$ and $\Delta \log g \approx \pm 0.1$, even if we include the "problematic" object HD 207198 mentioned above (this object, however, together with $\lambda \mathrm{Cep}$, does lie above the general trend).

It should be noted that some of the stars studied have deliberately been excluded from the analysis performed by Markova et al. (2003). Not only were the two binaries HD93129A and HD 303308 discarded but also the fast rotators (e.g., HD 217086 and HD 13268).

Nevertheless, the quality of the comparison performed is rather good with exception of the class V objects. For this luminosity class, Markova et al. have included the results obtained by Martins et al. (2002) into their calibrations. These results are based on an analysis of model-grids and on a different code (CMFGEN), which might lead to a certain inconsistency. The comparison of our results with the calibration now indicates a steeper relation, if we do not exclude the "outliers" HD 217086 and HD 15629 (both with $\log g \approx 3.7$ ). For both stars, the fit quality is very good, making an error in the spectroscopically derived $\log g$ very unlikely). To account for this problem. we have added an alternative regression based on our results only (along with the corresponding coefficients), denoted by the long-dashed line in Fig. 17.

If we assume, on the other hand, that the regression by Markova et al. were correct, the mismatch could be explained by means of an under-estimate of the true gravity, at least for HD $15629\left(V_{\mathrm{r}} \sin i=90 \mathrm{~km} \mathrm{~s}^{-1}\right)$ in case it were a fast rotator seen pole-on (Eq. (5)). For HD 217086, however, this possibility can most probably be excluded, since it is a fast rotator with only a small error in the centrifugal correction.

In conclusion, the $\log g$ vs. $T_{\text {eff }}$ calibration for 1.c.V objects remains somewhat uncertain, whereas for 1.c.I/III stars no obvious problems are visible.

\subsection{Is there still a mass discrepancy?}

Figure 18 shows the position of our objects in the HR Diagram, where different symbols have been used to mark objects with large He abundances or rotational velocities.

A comparison with the data from Paper I using the same absolute magnitudes (which would slightly modify the entries in Fig. 18) reveals two major differences which are explained by the new, lower temperatures and subsequently by the lower luminosities (remember that the radii are very similar).

First, lower masses are derived for the most massive stars, even if we include the binary components in Carina. In Paper I, progenitor masses in excess of $120 M_{\odot}$ and actual masses in excess of $100 M_{\odot}$ were derived for the most massive stars, whereas in our present work all stars have progenitor masses and actual masses below $100 M_{\odot}$.

Second, all of our stars are clearly separated from the ZAMS except HD 93128, although we have another five stars

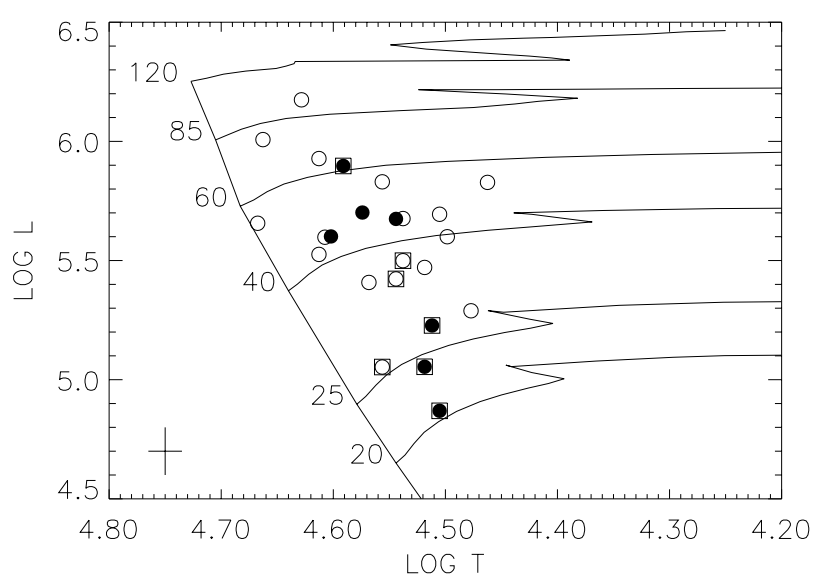

Fig. 18. The HR diagram for our sample. Filled symbols indicate stars with $\mathrm{He}$ abundances higher than $Y_{\mathrm{He}}=0.17$ and open squares mark the rapid rotators with $V_{\mathrm{r}} \sin i$ higher than $200 \mathrm{~km} \mathrm{~s}^{-1}$. Evolutionary tracks for non-rotating stars are taken from Schaller et al. (1992).

classified as dwarfs. This offset in effective temperature cannot be explained by distance uncertainties, unless they have been seriously overestimated (which would then pose a problem for HD 93128). A similar effect has been found in the data set analyzed by Herrero et al. (1992), although they have used different evolutionary models and there were no sample stars as young as HD 93128. Investigating the evolutionary tracks, this star would have an age of only $0.15 \mathrm{Myr}$ (see also Penny et al. 1993) which is much less than the age of the next youngest star, HD 93250 with an age of 1.3 Myr. Although the uncertainties in the derived ages are very large, this finding is consistent with the fact that both stars have very similar spectra and parameters ( $T_{\text {eff }}$ and $\log g$ ), but almost one and a half magnitudes difference in brightness. It is also consistent with the fact that HD 93128 is a member of Trumpler 14, which has been argued to be significantly younger than Tr 16 to which HD 93250 belongs (see Walborn 1982a, 1995). (Aside: we would like to point out that also HD 93250 might be a binary (cf. Walborn 1982b, but also Walborn et al. 2002) which would additionally explain the rather low wind-momentum rate of this star compared to similar objects.)

From Fig. 18 we can also read off the masses predicted by the evolutionary tracks (i.e., the evolutionary masses) and compare them with those derived from the stellar parameters which were determined by spectral analysis (i.e., the spectroscopic masses). Note that both masses depend in a similar way on the adopted distance, and, therefore, their difference will not change unless we have to modify this distance dramatically.

Herrero et al. (1992) performed this comparison and found that the spectroscopic masses of giants and supergiants were systematically smaller than the evolutionary ones. At the same time, the spectroscopically determined helium overabundances could not be explained by current theories of stellar structure and evolution. The correlation of the mass discrepancy, i.e., the difference between evolutionary and spectroscopic masses, and the distance to the Eddington limit indicated that the deduced discrepancy might have been related to the fact that Herrero et al. (1992) omitted sphericity and 


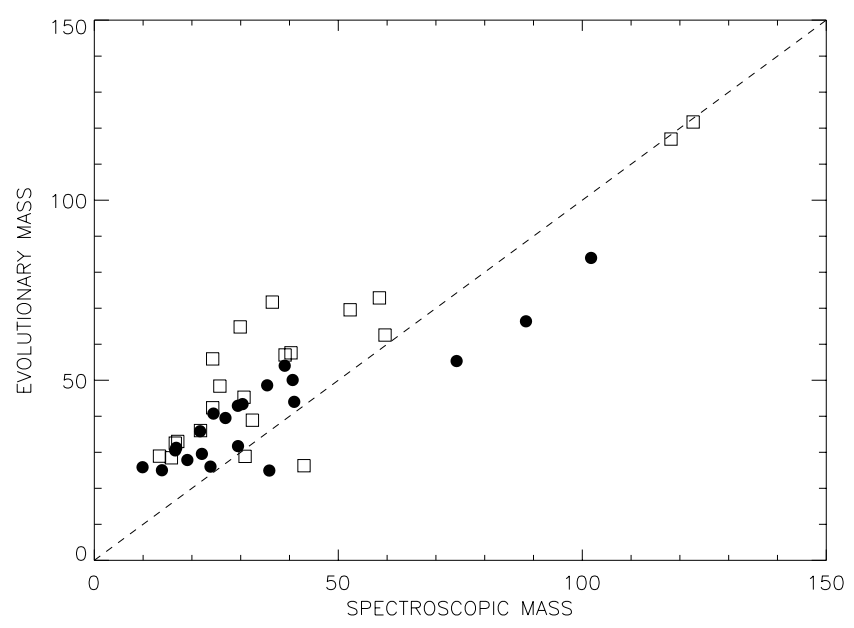

Fig. 19. Evolutionary vs. spectroscopic masses obtained in Paper I (open squares) and in the present work (filled circles), using the same absolute magnitudes ( $\rightarrow R_{\text {old }}$ ).

mass-loss in their analyses. However, even with the (approximate) inclusion of sphericity and mass-loss in the determination of stellar parameters as performed in Paper I, the actual problem could not been solved, but it could be improved.

From a recent analysis of seven Cyg OB2 supergiants including the effects of sphericity, mass-loss and line blanketing, Herrero et al. (2002) found no conclusive evidence for a mass discrepancy to be present. While the star with the lowest mass still showed a discrepancy, the other six stars were found to be evenly distributed on each side of the 1:1 line dividing spectroscopic and evolutionary mass, with the error bars crossing it.

In the present context and to illuminate the effects of line blocking/blanketing, it is now interesting to compare the evolutionary and spectroscopic masses from Paper I with the ones obtained in the present analysis, using the same absolute magnitudes. The result can be seen in Fig. 19. A distinct improvement of the general situation is obvious, especially for a number of stars in the "intermediate" mass range. Not surprisingly, these are stars for which the correction in effective temperature is very large.

The present situation (with respect to $M_{\mathrm{v}}$ and $R_{*}$ from Table 1) is displayed in Fig. 20. We see that for almost all stars the corresponding error bars cross the 1:1 line with exception of those three objects with the lowest spectroscopic mass (which are not the three objects with the lowest luminosity in Fig. 18).

Although our new results are consistent with the ones found by Herrero et al. (2002), implying that the mass discrepancy seems to be limited to stars of less than $15 M_{\odot}$, there is still additional concern. Most stars with masses lower than $50 M_{\odot}$ (including those three objects with mass discrepancy) follow an imaginary line located parallel to the 1:1 line which is displaced by about $10 M_{\odot}$ in ordinates. We consider this finding as a milder form of the original mass discrepancy which still has to be explained.

Unfortunately, is not possible to perform a similar comparison using evolutionary calculations based on rotating models as presented by Meynet \& Maeder (2000) and Heger \& Langer (2000), since we do not know the initial rotational velocity

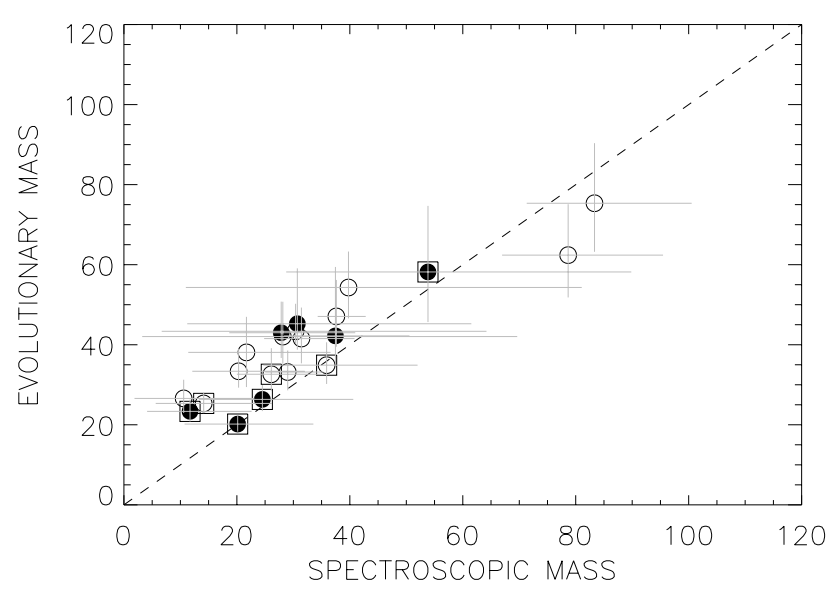

Fig. 20. Spectroscopic and evolutionary masses for our sample stars with data from Table 1 and errors as discussed in Sect. 6. The binary components HD 93129A and HD 303308 have been discarded from the plot. Open squares denote rapid rotators with $V_{\mathrm{r}} \sin i>200 \mathrm{~km} \mathrm{~s}^{-1}$, as in Fig. 18.

of our objects. If we simply assume that all our objects had started with $300 \mathrm{~km} \mathrm{~s}^{-1}$, the remaining discrepancies as discussed above would still have persisted. At least the derived range of $\mathrm{He}$ abundances could be explained, but not necessarily at the correct effective temperature, luminosity, and age as discussed by Herrero \& Lennon (2003).

\subsection{Wind-momentum rates}

Whereas the effective temperatures decrease significantly, mass-loss rates and stellar radii are hardly affected by lineblocking/blanketing and remain roughly at their "old" values (if the same values for distance $/ M_{\mathrm{v}}$ are used, of course). For the radii, this finding has already been explained in Sect 7.2 (increase of $T_{\text {rad }} / T_{\text {eff }}$ in the optical) and for the mass-loss rates the argument is similar: First, the $\mathrm{H}_{\alpha}$-emissivity increases only weakly due to the reduced electron temperature in the wind (at least for the hot stars considered here). Second, the underlying continuum, i.e., the radiation temperature close to $\mathrm{H}_{\alpha}$, remains comparable to or hotter than the continuum of an unblanketed model at its "older", higher $T_{\text {eff }}$. The combined effect of the modified electron, radiation and effective temperature on $\dot{M}$ can then be approximated by Eq. (49) in Paper I, and in most cases it turns out that we should expect a rather weak reduction in $\dot{M}$ of the order of 10 to $20 \%$.

\subsubsection{Comparison with previous results assuming identical $M_{v}$ 's}

This expectation has been checked in Fig. 21, where we have compared the modified wind-momentum rates as derived here with those resulting from Paper I. To avoid any confusion due to changes in $R_{*}$ because of differences in $M_{\mathrm{v}}$, this comparison has been performed on the basis of $R_{\text {old }}$ (Table 1, last column), i.e., assuming the same values for $M_{\mathrm{v}}$ as in Paper I. With respect to the values for luminosity and $D_{\text {mom }}$ as given in Tables 1 


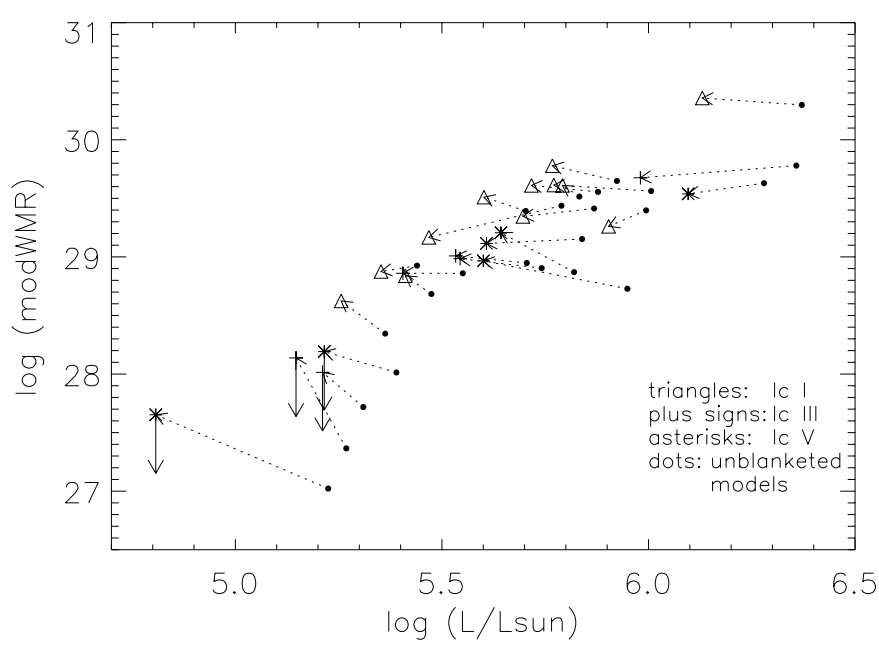

Fig. 21. Logarithm of modified wind-momentum rate, $D_{\text {mom }}=$ $\dot{M} v_{\infty}\left(R_{\star} / R_{\odot}\right)^{0.5}$, vs. $\log \left(L / L_{\odot}\right)$, for the values derived on the basis of $R_{\text {old }}$ (i.e., assuming the same values for $M_{\mathrm{v}}$ as in Paper I), compared to the results from Paper I itself. For all stars in our sample, a shift towards lower luminosities has been found, whereas for most of the stars the modified wind-momentum rate remains roughly constant. Only for the stars with $\log \left(L / L_{\odot}\right)<5.3$ the upper limit of the wind-momentum rate (indicated by arrows) has increased (see text).

and 4 , this means that both quantities have been scaled with $\left(R_{\text {old }} / R_{\star}\right)^{2}$.

In contrast to our expectation from above, Fig. 21 shows that in some cases we actually encounter an increase in $\dot{M}$. The reason for this behaviour is twofold. The fitted value of $\beta$ has decreased for a number of stars with emission lines, (e.g., for $\zeta$ Pup from 1.15 to 0.90 ), probably as a consequence of the somewhat modified run of $T_{\mathrm{e}}(r)$. A decrease in $\beta$ then translates into an increase in $\dot{M}$.

For objects with an almost purely photospheric $\mathrm{H}_{\alpha}$ profile, on the other hand (the four low luminosity stars for which we could obtain only upper limits), this upper limit has increased due to the higher precision of the present analysis: The approximate approach used in Paper I becomes somewhat uncertain at (very) low mass-loss rates, mainly because it depends on an incident boundary condition based on results from hydrostatic, plane-parallel models. Additionally, all four objects are very fast rotators. In Paper I, we reduced the rotational velocity from its photospheric value to a somewhat lower, "effective" value in order to match the $\mathrm{H}_{\alpha}$ profile. Again due to the higher precision of the present unified approach, it turned out that such a modification is no longer necessary, at least not for the three dwarfs (cf. Sects. 5 and 8). Keeping the nominal values of $V_{\mathrm{r}} \sin i$ then increases the derived mass-loss rates.

Insofar, the dilemma discussed in Paper I (What is the reason that the modified wind-momentum rates of low-luminosity stars lie below the average relation?) finds its natural explanation: For the present results, this dilemma simply no longer exists, at least if we assume that the actual mass-loss rates lie at the obtained upper limits (where this assumption, admittedly, is rather bold.)

From our findings, we conclude that for most of our objects the ratio between mass-loss rate (and thus modified windmomentum rate) and luminosity has become larger compared

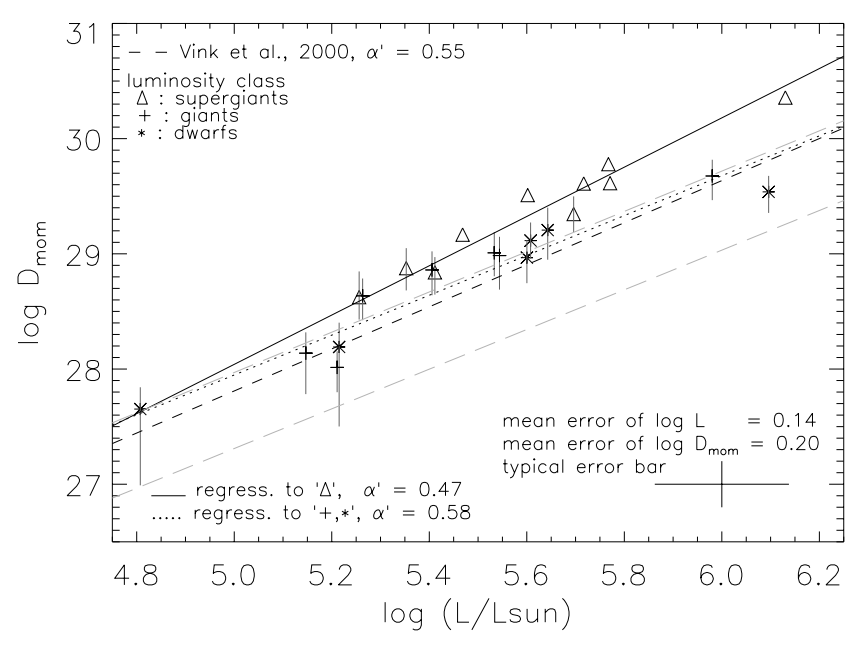

Fig. 22. WLR for our sample, using the same $M_{\mathrm{v}}$-values $\left(\rightarrow R_{\text {old }}\right)$ as in Paper I. Denotation as written in the plot legend. The binary components HD 93129A (1.c.I, at $\log L=6.13$ and $\log D_{\text {mom }}=30.35$ ) and HD 303308 (1.c.V, at $\log L=5.61$ and $\log D_{\text {mom }}=29.12$ ) have been discarded from the regressions. Error bars with respect to $\Delta \log D_{\text {mom }}$ are plotted only for objects with $\mathrm{H}_{\alpha}$ in absorption. The regression was performed accounting for the errors in both directions and for their correlation (see text). Overplotted (in grey, long-dashed) are the regressions as obtained in Paper I using pure $\mathrm{H} / \mathrm{He}$-models. Note that the new regression for luminosity classes III/V (dotted) almost coincides with the "old" regression for supergiants, and that both agree well with the predictions by Vink et al. (2000, dashed).

Table 5. Coefficients of the WLR obtained in the present investigation (discarding HD 93129A and HD 303308 from the regression), compared to the results from Paper I and the theoretical prediction by Vink et al. (2000). Entry 4 and 5 correspond to values obtained by using the "old" absolute magnitudes ( $\rightarrow R_{\text {old }}$ ), entry 6 and 7 correspond to the values derived from new ones including the results for seven Cyg OB2 stars by Herrero et al. (2002). The last entry corresponds to the regression performed in Fig 24. Present data has been analyzed by accounting for the errors in both directions and their correlation, whereas a standard least square fit has been performed for the data in Paper I (no errors available).

\begin{tabular}{llllc}
\hline \hline \multicolumn{2}{c}{ Sample } & \multicolumn{1}{c}{$\log D_{\mathrm{o}}$} & \multicolumn{1}{c}{$x$} & $\alpha^{\prime}$ \\
\hline Vink et al. (2000) & $18.68 \pm 0.26$ & $1.83 \pm 0.044$ & $0.55 \pm 0.013$ \\
sg & (Paper I) & $19.23 \pm 0.98$ & $1.75 \pm 0.17$ & $0.57 \pm 0.055$ \\
g/d & (Paper I) & $18.90 \pm 1.46$ & $1.72 \pm 0.25$ & $0.58 \pm 0.085$ \\
sg & $M_{\mathrm{v}}$ (old) & $17.34 \pm 2.46$ & $2.14 \pm 0.44$ & $0.47 \pm 0.096$ \\
g/d & $M_{\mathrm{v}}$ (old) & $19.3 \pm 1.22$ & $1.73 \pm 0.22$ & $0.58 \pm 0.074$ \\
sg & (+CygOB2) & $17.98 \pm 1.88$ & $2.00 \pm 0.32$ & $0.50 \pm 0.080$ \\
g/d & (+CygOB2) & $18.70 \pm 1.29$ & $1.84 \pm 0.23$ & $0.54 \pm 0.068$ \\
"unified", cf. Fig. 24 & $18.92 \pm 0.87$ & $1.80 \pm 0.16$ & $0.56 \pm 0.049$ \\
\hline
\end{tabular}

to previous results. This becomes particularly clear in Fig. 22, where we compare the average WLR for Galactic supergiants and giants/dwarfs obtained in Paper I (long-dashed, grey) with the corresponding regressions using the present data, again for the same values of $M_{\mathrm{v}}$. Note that the latter have been performed accounting for the errors in both directions ( $\Delta \log L$ and $\Delta \log D_{\text {mom }}$, cf. Table 4) and for their correlation (both quantities depend on $R_{\star}^{2}$ ), as described in Markova et al. (2003). 
The corresponding coefficients with respect to the WLR,

$$
\log D_{\text {mom }}=x \log \left(L / L_{\odot}\right)+\log D_{\mathrm{o}}, \quad x=\frac{1}{\alpha^{\prime}},
$$

(with $\alpha^{\prime}$ being the exponent of the line-strength distribution function, corrected for ionization effects) are given in Table 5. Interestingly, the new regression for luminosity classes III/V (dotted) almost coincides with the "old" regression for supergiants, whereas the new regression for the supergiants has become significantly steeper than previously determined. Taken literally, the new value of $\alpha^{\prime}(\mathrm{sg})$ is smaller than $\alpha^{\prime}(\mathrm{g} / \mathrm{dw})$, in contrast to results from theoretical considerations (Puls et al. 2000). Let us point out, however, that the regression coefficients for supergiants are rather uncertain, since the minimum of $\chi^{2}$ is extremely broad (in contrast to the lc III/V case).

Although quantitatively different, both the results presented here and in Paper I indicate a clear separation between luminosity class I objects and the rest, although this separation seems to have decreased regarding our new data. The most obvious interpretation would be that the effective number of lines driving the wind (comprised in the quantity $D_{\mathrm{o}}$ from Eq. 16) is a function of luminosity class. A comparison with recent theoretical predictions (and even with older ones, cf. Fig. 25 in Paper I) obtained by different independent approaches (Vink et al. 2000; Pauldrach et al. 2003; Puls et al. 2003a) suggests that this is not probable, since these calculations predict a unique relation, almost coinciding with our present regression for class III/ $\mathrm{V}$ objects (cf. Figs. 22, 23).

\subsubsection{Clumping in the lower wind?}

If we now use our preferred absolute magnitudes, i.e., the values given in Tables 1 and 4 and include the results obtained by Herrero et al. (2002) for seven Cyg OB2 stars $^{11}$ (which should be free of errors related to relative distances), the situation becomes even more confusing as shown in Fig. 23 (see also Puls et al. 2003a, Fig. 3). Although the Cyg OB2 sample consists almost exclusively of supergiants, only the two most extreme supergiants (CygOB2\#7 and \#11) follow the "upper" WLR from Fig. 22, whereas the derived wind-momenta for all other (five) objects are consistent with our present WLR for class III/V stars.

In order to clarify this confusion and on the basis of a preliminary analysis of the present data set, Puls et al. (2003a) have suggested a scenario which we would like to briefly summarize, because in the following we will present the corresponding outcome using our results.

When plotted not as a function of luminosity class but as a function of $\mathrm{H}_{\alpha}$ profile type (Puls et al. 2003a, Fig. 4), it turned out that stars with $\mathrm{H}_{\alpha}$ in emission and those with absorption profiles (only partly filled in by wind emission) form two distinct WLRs, respectively, where in this representation the CygOB2 objects make no exception.

\footnotetext{
11 Note that this analysis has been performed with the same code as applied by us, i.e., the results are at least in a differential sense of equal quality.
}

From these findings, Puls et al. (2003a) suggested that the different WLRs might be a consequence of wind-clumping: The contribution of wind emission to the total profile is significantly different for objects with $\mathrm{H}_{\alpha}$ in absorption compared to object with $\mathrm{H}_{\alpha}$ in emission, since for the former only contributions from the lowermost wind can be seen, whereas for the latter the emission is due to a significant wind volume. Thus, there is the possibility that for these objects we see the effects of a clumped wind which would mimic a higher mass-loss rate, as it is most probably the case for Wolf-Rayet winds (e.g., Moffat $\&$ Robert 1994). With this suggestion, we do not exclude the presence of clumping in the winds of objects with $\mathrm{H}_{\alpha}$ in absorption; owing to the low optical depth, however, we simply cannot see it.

It should be mentioned that the principal presence of clumping has never been ruled out for O-star winds; however, at least from conventional spectrum analysis methods there was simply no indication that the $H_{\alpha}$ forming region was considerably clumped (see the discussion in Paper I). During the past years, this situation has somewhat changed. Apart from recent theoretical considerations (e.g., Feldmeier et al. 1997; Owocki \& Puls 1999 and references therein) which do not prohibit such a relatively deep-seated clumped region, a number of additional evidence for such a scenario has been gathered.

First, note that time-series analyses of He II 4686 from $\zeta$ Pup by Eversberg et al. (1998) have revealed "outward moving inhomogeneities" from regions near the photosphere out to $2 R_{*}$, i.e., just in the $\mathrm{H}_{\alpha}$ forming region which extends typically out to 1.5 stellar radii. Although these features are most probably different from the clumps suggested here, these observations indicate that the lower wind is not as stationary as previously assumed.

Second, our hypothesis is supported by a number of UVanalyses. Based on FUSE-observations of (L)MC-stars, both Crowther et al. (2002), Massa et al. (2003) and Hillier et al. (2003, see below) found indications that the winds might be clumped, majorly from the behaviour of the PV resonance line (if phosphorus is not strongly under-abundant, as claimed by Pauldrach et al. 1994). By comparing the results from selfconsistent wind models and UV line-synthesis, Puls et al. (2003a) found that the clumping scenario is also consistent with the behaviour of the SiIV resonance line.

Most important in the present context, however, is the problem concerning the cores of the (blue) Balmer lines discussed in Sect. 4. At least for five out of the seven objects with $\mathrm{H}_{\alpha}$ in emission, the synthetic $\mathrm{H}_{\gamma} \mathrm{H}_{\delta}$ (where present) and $\mathrm{H}_{\beta}$ lines formed in or close to the photosphere show too much wind emission in their cores, and would require at least a factor of 1.5 less mass-loss in order to be consistent with observations. Interestingly, this behaviour has also been found for the extreme objects of the Cyg OB2 sample analyzed by Herrero et al. (2002). This dilemma is not present for stars with $\mathrm{H}_{\alpha}$ in absorption for which we anticipate that the derived mass-loss rates are not contaminated by clumping effects.

In Fig. 24 we have considered the following question: If the suggested scenario was correct, what (clumping) factor would be required to "unify" the different WLRs with each other and with the theoretical predictions, respectively? To this end, we 


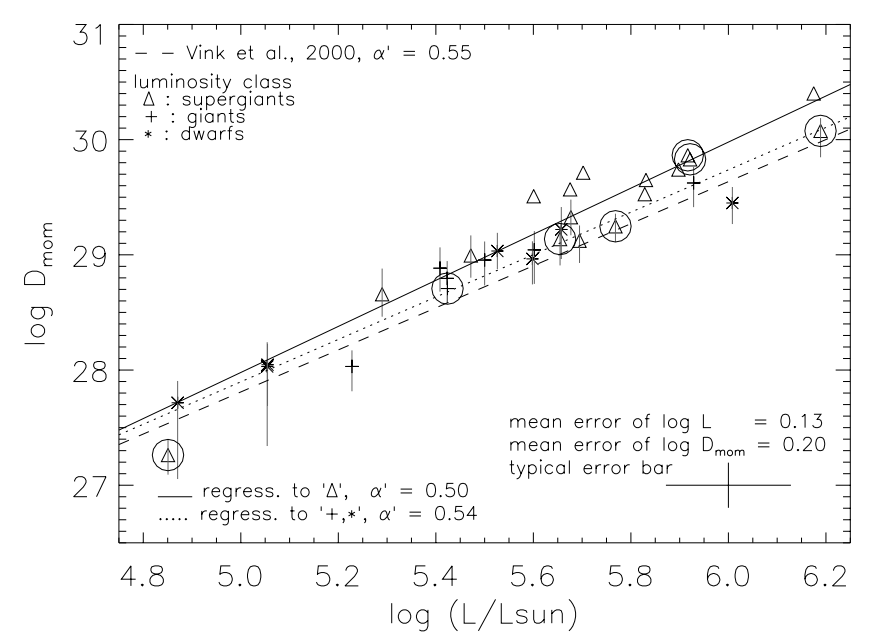

Fig. 23. As Fig. 22, but with the actual absolute magnitudes from Table 1 including the results for seven Cyg OB2 stars analyzed by Herrero et al. (2002) (enclosed by circles).

have modified the mass-loss rates for all objects with $\mathrm{H}_{\alpha}$ in emission (including the $\mathrm{CygOB} 2$ stars) in order to match the WLR of giants/dwarfs as close as possible. This approach, of course, assumes that either the clumping factor in the $\mathrm{H}_{\alpha}$ emitting wind region is constant or that the $\mathrm{H}_{\alpha}$ emitting volume is of similar size. The required factor with respect to $\dot{M}$ turned out to be 0.44 , corresponding to an (effective) clumping factor $<\rho^{2}>/<\rho>^{2}=0.44^{-2}=5.2$, which sounds reasonable and is somewhat lower than the values inferred from (the outer regions of) Wolf-Rayet winds.

With such a reduction we find an almost unique WLR consistent with theoretical simulations ${ }^{12}$, where the corresponding parameters are given in Table 5. Recall that the quoted values have been "derived" on the basis of the hypothesis that the modified wind-momentum rate is a function of luminosity alone, independent of luminosity class. This hypothesis is strongly supported by theoretical predictions and simulations in those cases where the WLR is independent of stellar mass (which is the case for Galactic O-stars with $\alpha^{\prime}$ close to 2/3). If this hypothesis is wrong, the derived clumping factors might be somewhat too large.

In any case and in summary, there are strong indications that mass-loss analyses of (at least) O-star winds utilizing $\mathrm{H}_{\alpha}$ tend to overestimate the resulting values, unless clumping is accounted for or the winds are comparatively thin.

After finishing this investigation a very interesting paper by Hillier et al. (2003) appeared which gave additional support to our hypothesis. In this paper, two SMC O stars, AV83 (O7 Iaf $\left.{ }^{+}\right)$and AV69 (OC7.5 III((f))) have been analyzed by means of CMFGEN. Although both objects are shown to be located at rather similar effective temperatures and luminosities $\left(\log \left(L / L_{\odot}\right)=5.54\right.$ and 5.62, respectively), their spectra display quite different wind signatures, with $\mathrm{H}_{\alpha}$ in emission for the supergiant and in absorption for the giant. Whereas for the supergiant the mass-loss rate could be determined precisely

\footnotetext{
${ }^{12}$ For the "outliers" around $\log \left(L / L_{\odot}\right) \approx 5.8$, the deduced factor might be too large, indicating that these stars are affected by a smaller clumped wind volume than the rest.
}

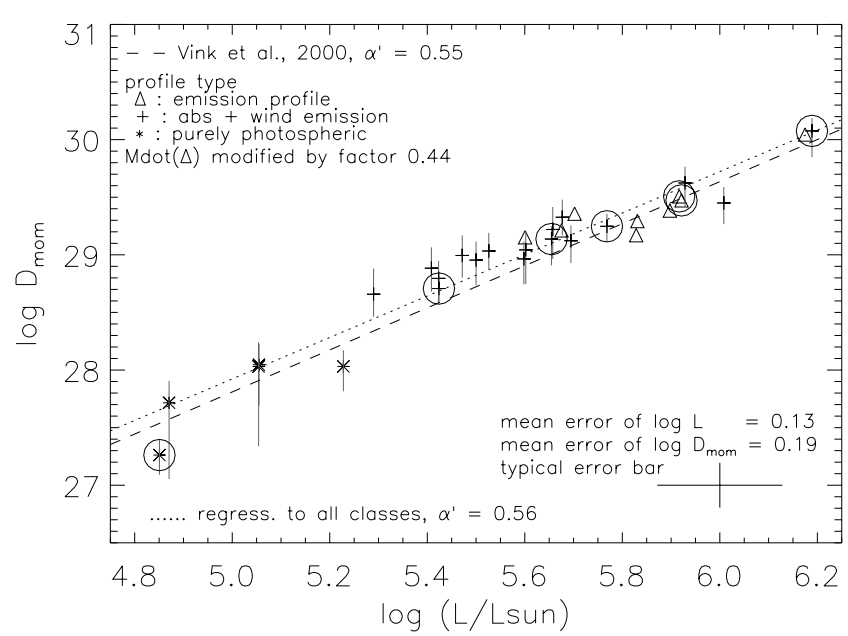

Fig. 24. WLR of the combined sample from Fig. 23, $\dot{M}$ for objects with $\mathrm{H}_{\alpha}$ in emission reduced by a factor of 0.44 . The resulting WLR follows closely the predictions by Vink et al. (2000) (see also Markova et al. 2003).

( $\dot{M}=2.0 \times 10^{-6} M_{\odot} /$ yr for $\beta=2$, neglecting clumping), the giant's mass-loss rate could not be derived unambigously, due to the $\beta$-problem discussed in Sect. 6.2. For $\beta=0.7,1.0,2.0$ massloss rates of $\dot{M}=1.5,0.92$ and $0.32 \times 10^{-6} M_{\odot} /$ yr are quoted, respectively. Accounting for the different terminal velocities (960 $\mathrm{km} \mathrm{s}^{-1}$ vs. $1800 \mathrm{~km} \mathrm{~s}^{-1}$ - strongly related to the different gravities), the derived modified wind-momentum rates are quite similar if clumping is neglected. For an assumed value of, e.g., $\beta=1.0$ for the giant, $D_{\text {mom }}$ for the less luminous supergiant is slightly larger, by a factor of only 1.16 .

On various evidence (including the behaviour of the PV line, see above), the authors then argue that the supergiant wind is probably clumped, and that the clumping should begin at the base of the wind! In this case, for a best simultaneous fit of all photospheric and wind lines the mass-loss rate becomes reduced by a factor of 0.37 . Note that this number as well as the conclusion of deep-seated clumping agrees very well with our above hypothesis. Note also that no clumping correction has been applied to the giant, since clumping is (if at all) only weakly visible in a small number of UV wind lines (OIV and CIV), such that a correction of $\dot{M}$ would be less than for the supergiant. Taking all numbers literally, a "unification" of the wind-momentum rates in the same spirit as above (i.e., claiming an equal value of $D_{\mathrm{o}}$ in Eq. (16)) would yield a value of $\alpha^{\prime} \approx 0.2$ for $\beta$ (giant) $=1.0$, which might be somewhat low for SMC O-(super)giants (cf. Puls et al. 2000; Vink et al. 2001). Note, however, that only a small reduction of the giant's massloss rate to a value of $0.58 \times 10^{-6} M_{\odot} / \mathrm{yr}$ (corresponding to a $\beta \approx 1.5$ or/and a moderately clumping corrected mass-loss rate) would yield a much more typical value, i.e., $\alpha^{\prime} \approx 0.4$.

\section{Conclusions and summary}

In this paper, we have re-analyzed the Galactic O-star sample from Paper I by means of line-blanketed NLTE model atmospheres in order to investigate the influence of lineblocking/blanketing on the derived stellar and wind parameters. 
For our analysis and in addition to the "conventional" strategic lines (e.g., Herrero et al. 1992), we have included a number of He lines neighboring $\mathrm{H}_{\alpha}$ to provide complementary constraints on the fitting procedure.

The fit quality is generally good (or even very good), except for the following systematic inconsistencies:

i) For five out of seven supergiants with $\mathrm{H}_{\alpha}$ in emission, the line cores of the blue Balmer lines are too weak (i.e., too much filled in by wind emission) when the mass-loss rate was determined by matching $\mathrm{H}_{\alpha}$. This effect (in conjunction with the analysis of the WLR) might indicate an overestimate in mass-loss rate.

ii) In a number of cases, He II 4541 turned out to be too weak, although He II 4200 (same lower level!) fitted perfectly. There are two possibilities to explain this deficiency: either the upper level is too strongly populated, or the wind emission is too large (note, that He II 4541 is stronger than He II 4200, i.e., is formed further out in the wind). Since in most cases this problem occurred in parallel with the problem outlined in item i), a relation to an erroneous mass-loss rate cannot be excluded. Interestingly, Herrero et al. (1992) (using plane-parallel models) have reported a similar inconsistency, but in their case He II 4200 was too weak.

iii) Although our models comprise the effects of mass-loss, sphericity and blanketing, with the present version of FASTWIND we were not able to get rid of the "generalized dilution effect" in He I 4471 emanating from both cooler supergiants and giants. The onset of this effect was found to be located around spectral type O6. For almost all affected stars the blue Balmer lines could perfectly be fitted, which makes a relation to an overestimated mass-loss rate rather unlikely.

iv) If $\mathrm{H}_{\alpha}$ appears with a $\mathrm{P}$ Cygni shape, we are not able to match the blue absorption component. This finding points either to an inconsistent treatment of the corresponding $\mathrm{He}$ II blend or to effects of stellar rotation disturbing the emergent profile.

v) In Paper I, the nominal value of $V_{\mathrm{r}} \sin i$ (from photospheric lines) was reduced for most of the objects with a large rotational velocity in order to match the observed $\mathrm{H}_{\alpha}$ profiles. This was explained by the fact that the wind emission is formed in a differentially rotating medium with an "effective" rotational speed smaller than the photospheric one (see also Petrenz \& Puls 1996). In our present analysis, we have encountered the same effect, but only in giants (and one supergiant), namely in HD 18409, HD 193682, HD 24912, HD 203064 and HD 191423 (cf. Sect. 5). For the fast-rotating dwarfs (HD 217086, HD 13268 and HD 149757), on the other hand, no discrepancy between $\mathrm{H}_{\alpha}$ and the other lines was detected, in contrast to the results from Paper I. Actually, this finding is quite reassuring, since the wind-emission in those stars is so low that an influence of differential rotation in the wind $\left(V_{\text {rot }}(r) \propto V_{\text {rot }}\left(R_{\star}\right) R_{\star} / r\right)$ is rather unlikely.

On the other hand, it should be noted that in most cases the notorious wind line He II 4686 could be reproduced in parallel with $\mathrm{H}_{\alpha}$, indicating that our (approximate) treatment of lineblocking around He II 303 is rather accurate.

Compared to pure $\mathrm{H} / \mathrm{He}$ plane-parallel models, our new results display the following trend:

For a given spectral type, the effective temperatures become lower along with a reduction of either gravity or helium abundance. The reduction of $T_{\text {eff }}$ is largest at earliest spectral types and for supergiants, and decreases towards later types. At 09.5, the differences to unblanketed analyses are small, at least for the objects from our sample. Recent work by Crowther et al. (2002), however, indicates that extreme O-supergiants of late spectral types have significantly lower values of $T_{\text {eff }}$ than our objects.

The reduction of the effective temperature scale has been explained in Sect. 7.2 as the final consequence of UV lineblocking, increasing both the electron temperature by lineblanketing and the mean intensity by back-scattering in the outer photosphere where the strategic lines are formed. As a result, line-blanketed models of hot stars have photospheric $\mathrm{He}$ ionization fractions similar to those from unblanketed models at higher $T_{\text {eff }}$ and higher $\log g$. Thus, any analysis based on the He ionization equilibrium results in lower values of $T_{\text {eff }}$, if lineblocking/blanketing is accounted for. In those cases, where a reduction of $\log g$ is prohibited by the Balmer line wings, the helium abundance has to be reduced instead in order to allow for a convincing fit.

On the other hand, stellar radii and mass-loss rates (and hence modified wind-momentum rates) remain roughly unaffected by line-blanketing, since the optical fluxes from lineblanketed models are similar to those from unblanketed models at their corresponding, higher effective temperatures due to flux-conservation.

After correcting for the centrifugal acceleration (where this correction and the corresponding error has been derived in Appendix A), we calculated the masses and compared them with previous results as well as with evolutionary masses. Although the former mass discrepancy (Herrero et al. 1992) becomes significantly reduced, it still seems to exist at the lowest masses $\left(M<15 M_{\odot}\right)$. For all stars with larger mass, at least the corresponding error bars do cross the 1:1 line. Nevertheless, a systematic trend seems to be present: For $15 M_{\odot}<M<$ $50 M_{\odot}$, the spectroscopically derived values are still lower than the masses derived from evolutionary calculations by roughly $10 M_{\odot}$.

In the case of the helium discrepancy, we could significantly reduce the He abundance for a number of objects, particularly for those where previous values were extremely large (e.g., for HD $193682 Y_{\mathrm{He}}$ has decreased from 0.43 to 0.20 ). A significant fraction of our sample stars, however, still remains over-abundant in He. For these objects, at least the derived range in abundance is consistent with present evolutionary tracks when rotationally induced mixing is accounted for.

One of the major implications of reduced luminosities and almost unaltered wind-momentum rates affects the windmomentum luminosity relation. Previous results for O-stars (Puls et al. 1996; Kudritzki \& Puls 2000) indicated a clear separation of the WLR as function of luminosity class, where the 
WLR for supergiants was found to be more or less consistent with recent theoretical simulations which do not predict any dependence on luminosity class. For giants and dwarfs, the WLR was located roughly 0.5 dex below that. In addition, it showed a kink towards even lower momentum rates for objects with $\log \left(L / L_{\odot}\right)<5.3$ which could not be explained so far.

Regarding our new values, the separation of the WLR is still present with one decisive difference. Now, the WLR for giants/dwarfs is consistent with theoretical expectations and also the kink has vanished at least if we assume that the actual massloss rates lie at the obtained upper limits. On various evidence (including recent UV-analyses and the problematic line cores of the blue Balmer lines, see above, with additional support from a recent investigation by Hillier et al. 2003) we have argued that the different WLRs can be unified on the basis of the following assumptions: For those stars with $\mathrm{H}_{\alpha}$ in emission, the derived mass-loss rates are affected by clumping in the lower wind region. For stars with $\mathrm{H}_{\alpha}$ in absorption, on the other hand, this line is formed very close to the photosphere so clumping effects cannot disturb the analysis. This kind of unification would then require a clumping factor of roughly 5 , where the mass-loss rates of stars with $\mathrm{H}_{\alpha}$ in emission would typically be overestimated by a factor of 2.3. As displayed in Fig. 24, the combined WLR is then consistent also with theory.

We have, of course, to be open to other possibilities which might explain the discrepancies found. A combined multispectral analysis (UV, optical, IR and radio) based on clumped wind-models and applied to large samples of stars of different spectral type should clarify these questions as well as others, e.g., the problem of wind-momenta from mid-type B-supergiants which appear to be much lower than expected (Kudritzki et al. 1999).

One of the major problems encountered in the present analysis is the uncertainty in stellar radius, which originates from uncertain distances and enters quadratically into the values for masses, luminosities and wind-momentum rates.

Since the distances to Galactic O-stars (which are important because of their "Galactic" abundances) will probably pose a problem for the next decade(s), there are only two possibilities to overcome this uncertainty (at least indirectly). Either we consider samples much larger than the present one (with the hope that better statistics will help to obtain better constraints), or we concentrate on the analysis of O-stars (definitely) belonging to distinct clusters (with the hope that the analysis is at least intrinsically consistent).

For recent progress into direction "one", we refer the reader to Markova et al. (2003). Regarding the second possibility, a first step has been taken by Herrero et al. (2002), although the amount of analyzed objects (seven) is not enough to obtain representative results. Observational campaigns utilizing multiobject spectroscopy like the upcoming FLAMES-project, aiming at the analysis of samples of more than hundred Extragalactic and Galactic objects, will definitely lead to a dramatic increase of our knowledge of hot, massive stars.

Acknowledgements. T.R. gratefully acknowledges financial support in form of a grant by the International Max-Planck Research School on Astrophysics (IMPRS), Garching. Many thanks especially to the anonymous referee of this paper as well as to Dr. Mark Neeser and Dr. Nevyana Markova for useful comments on the manuscript. We would also like to thank Dr. Nolan Walborn for his suggestions, especially for drawing our attention to the importance of the Lutz-Kelker effect. This work has been partially supported by the Spanish MCyT under project PNAYA 2001-0436.

\section{References}

Abbott, D. C., \& Lucy, L. B. 1985, ApJ, 288, 679

Bohannan, B., Voels, S. A., Hummer, D. G., \& Abbott, D. C. 1990, ApJ, 365, 729

Bromm, V., Kudritzki, R. P., \& Loeb, A. 2001, ApJ, 552, 464

Chandrasekhar, S., \& Münch, G. 1950, ApJ, 111, 142

Cranmer, S. R., \& Owocki, S. P. 1995, ApJ, 440, 308

Crowther, P. A., Hillier, D. J., Evans, C. J., et al. 2002, ApJ, 579, 774

de Zeeuw, P. T., Hoogerwerf, R., de Bruijne, J. H. J., et al. 1999, AJ, 117,354

ESA 1997, The Hipparcos and Tycho Catalogues, ESA SP-1200

Eversberg, T., Lepine, S., \& Moffat, A. F. J. 1998, ApJ, 494, 799

Feldmeier, A. 1995, A\&A, 299, 523

Feldmeier, A., Puls, J., \& Pauldrach, A. W. A. 1997, A\&A, 322, 878

Gabler, R., Gabler, A., Kudritzki, R. P., Puls, J., et al. 1989, A\&A, 226,162

Garmany, C. D., \& Stencel, R. E. 1992, A\&ASS, 94, 211

Gräfener, G., Koesterke, L., \& Hamann, W.-R. 2002, A\&A, 387, 244

Harries, T. J., \& Howarth, I. D. 1996, A\&A, 310, 533

Haser, S. M. 1995, Ph.D. Thesis, Universität München

Heger, A., \& Langer, N. 2000, ApJ, 544, 1016

Herrero, A. 1993, SSRv, 66, 137

Herrero, A., \& Lennon, D. J. 2003, in Proc. IAU Symp., 215, ed. A. Maeder, \& P. Eenens, ASP, in press

Herrero, A., Kudritzki, R. P., Vílchez, J. M., et al. 1992, A\&A, 261, 209

Herrero, A., Puls, J., \& Villamariz, M. R. 2000, A\&A, 354, 193

Herrero, A., Puls, J., \& Najarro, F. 2002, A\&A, 396, 949

Hillier, D. J., \& Miller, D. L. 1998, ApJ, 496, 407

Hillier, D. J., Lanz, T., Heap, S. R., et al. 2003, ApJ, 588, 1039

Hoogerwerf, R., de Bruijne, J. H. J., \& de Zeeuw, P. T. 2001, A\&A, 365,49

Howarth, I. D., Siebert, K. W., Hussain, G. A. J., et al. 1995, MNRAS, 284,265

Hubeny, I., \& Lanz, T. 1995, ApJ, 39, 875

Hubeny, I., Heap, S. R., \& Lanz, T. 1998, ASP Conf. Ser., 131, 108

Hummer, D. G. 1982, ApJ, 257, 724

Hummer, D. G., Abbott, D. C., Voels, S. A., \& Bohannan, B. 1988, ApJ, 328, 704

Humphreys, R. M. 1978, ApJSS, 38, 309

Koen, C. 1992, MNRAS, 256, 65

Kudritzki, R. P. 1980, A\&A, 85, 174

Kudritzki, R. P., \& Puls, J. 2000, ARA\&A, 38, 613

Kudritzki, R. P., Lennon, D. J., \& Puls, J. 1995, in Quantitative Spectroscopy of Luminous Blue Stars in Distant Galaxies. ESO Astrophysics Symposia, Science with the VLT, ed. J. R. Walsh, \& I. J. Danziger (Heidelberg: Springer), 246

Kudritzki, R. P., Puls, J., Lennon, D. J., et al. 1999, A\&A, 350, 970

Lennon, D. J., Dufton, P. L., \& Fitzsimmons, A. 1992, A\&AS, 94, 569

Lennon, D. J., Dufton, P. L., \& Fitzsimmons, A. 1993, A\&AS, 97, 559

Leitherer, C., \& Heckman, T. M. 1995, ApJS, 96, 9

Lutz, T. E., \& Kelker, D. H. 1973, PASP, 85, 573

Maeder, A., \& Meynet, G. 2000, ARA\&A, 38, 143

Maeder, A., \& Meynet, G. 2000, A\&A, 361, 159 
Markova, N., Puls, J., Repolust, T. M., \& Markov, H. 2003, in press

Martins, F., Schaerer, D., \& Hillier, D. J. 2002, A\&A, 382, 999

Massa, D., Fullerton, A. W., Sonneborn, G., et al. 2003, ApJ, 586, 996

Massey, P. 1998, VIII IAC Winter School of Astrophysics: Stellar Astrophysics for the Local Group, ed. A. Aparicio, A. Herrero, \& F. Sanchez, CUP, 95

Meynet, G., \& Maeder, A. 2000, A\&A, 361, 101

Moffat, A. F. J., \& Robert, C. 1994, ApJ, 421, 310

Nelan, E., et al. 2003, in prep.

Oey, S. 2003, in Proc. IAU Symp. 212, ed. K. A. van der Hucht, A. Herrero, \& C. Esteban, ASP, 620

Oudmajier, R. D., Groenewegen, M. A. T., \& Schrijver, H. 1998, MNRAS, 294, L41

Owocki 1999, in Proc. IAU Coll. 169, ed. B. Wolf, O. Stahl, \& A. W. Fullerton, 294

Owocki, S. P., \& Puls, J. 1999, ApJ, 510, 355

Owocki, S. P., Castor, J. I., \& Rybicki, G. B. 1988, ApJ, 335, 914

Pauldrach, A. W. A., Puls, J., \& Kudritzki, R. P. 1986, A\&A, 164, 86

Pauldrach, A. W. A., Kudritzki, R. P., Puls, J., et al. 1994, A\&A, 283, 525

Pauldrach, A. W. A., Hoffmann, T. L., \& Lennon, M. 2001, A\&A, 375, 161

Pauldrach, A. W. A., Hoffmann, T. L., \& Méndez, R. 2003, in Proc. IAU Symp., 209, ed. S. Kwok, \& M. Dopita, in press

Penny, L. R. 1996, ApJ, 463, 737

Penny, L. R., Gies, D. R., Hartkopf, W. I., et al. 1993, PASP, 105, 588

Petrenz, P., \& Puls, J. 1996, A\&A, 312, 195

Petrenz, P., \& Puls, J. 2000, A\&A, 358, 956

Pettini, M., Steidel, C. C., Adelberger, K. L., et al. 2000, ApJ, 528, 96

Puls, J., Kudritzki, R. P., Herrero, A., et al. 1996, A\&A, 305, 171 (Paper I)

Puls, J., Springmann, U., \& Lennon, M. 2000, A\&A, 141,23
Puls, J., Repolust, T., Hoffman, T. L., et al. 2003, in Proc. IAU Symp., 212, ed. K. A. van der Hucht, A. Herrero, \& C. Esteban, ASP, 61 Puls, J., et al. 2003, in prep.

Santolaya-Rey, A. E., Puls, J., \& Herrero, A. 1997, A\&A, 323, 488

Silich, S., \& Tenorio-Tagle, G. 2001, ApJ, 552, 9

Schaerer, D., \& Schmutz, W. 1994, A\&A, 288, 231

Schaller, G., Schaerer, D., Meynet, G., \& Maeder, A. 1992, A\&AS, 96, 269

Schmutz, W. 1991, in Stellar Atmospheres: Beyond Classical Models, ed. L. Crivellari, I. Hubeny, \& D. G. Hummer, NATO ASI Series C, 341 (Dordrecht: Kluwer), 191

Schmutz, W. 1997, A\&A, 321, 268

Shortridge, K. 1987, FIGARO user manual

Smith, K. C., \& Howarth, I. D. 1998, MNRAS, 299, 1146

Steidel, C. C., Giavalisco, M., Pettini, M., et al. 1996, ApJL, 462, L17

Urbaneja, M. A., Herrero, A., Bresolin, F., et al. 2003, ApJL, 584, 73

Vacca, W. D., Garmany, C. D., \& Shull, M. 1996, ApJ, 460, 914

Villamariz, M. R., \& Herrero, A. 2000, A\&A, 357, 597

Villamariz, M. R., Herrero, A., Butler, K., \& Becker, S. R. 2002, A\&A, 388, 940

Vink, J. S., de Koter, A., \& Lamers, H. J. G. L. M. 2000, A\&A, 362, 295

Vink, J. S., de Koter, A., \& Lamers, H. J. G. L. M. 2001, A\&A, 369, 574

Voels, S. A., Bohannan, B., \& Abbott, D. C. 1989, ApJ, 340, 1073

Walborn, N. R. 1972, AJ, 77, 312

Walborn, N. R. 1973, AJ, 78, 1067

Walborn, N. R. 1982a, AJ, 87, 1300

Walborn, N. R. 1982b, ApJ, 254, L15

Walborn, N. R. 1995, RMxAC, 2, 51

Walborn, N. R., Howarth, I. D., Lennon, D. J., et al. 2002, AJ, 123, 2754 
T. Repolust et al.: Stellar and wind parameters of Galactic O-stars, Online Material p 1

\section{Online Material}




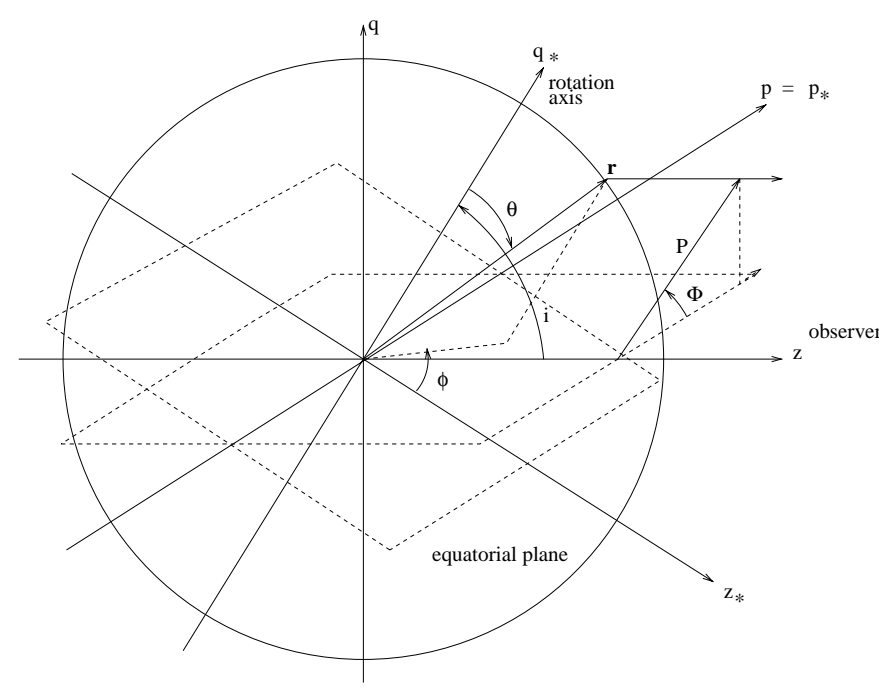

Fig. A.1. Geometry used for calculating the centrifugal correction (see text).

\section{Appendix A: Centrifugal correction}

The objective of this appendix is to derive an expression for the centrifugal correction which we will apply to the "effective" gravity, in order to obtain an approximation for the "true" value and the corresponding error (cf. Eqs (3), (4)). Thus, we have to evaluate the average

$\left\langle g_{\text {cent }}\right\rangle_{\text {disk }}=\frac{\left\langle\left(V_{\text {rot }} \sin \theta\right)^{2}\right\rangle}{R_{\star}}$,

where $\left\langle g_{\text {cent }}\right\rangle_{\text {disk }}$ is averaged is over the stellar disk and, thus, depends on the inclination $\sin i$. This quantity must not be mistaken for the centrifugal acceleration averaged over the stellar sphere,

$\left\langle g_{\text {cent }}\right\rangle_{\text {sphere }}=\frac{\int_{0}^{2 \pi} \int_{0}^{\pi} g_{\text {cent }}(\theta) R_{\star}^{2} \sin \theta \mathrm{d} \theta \mathrm{d} \phi}{4 \pi R_{\star}^{2}}=\frac{2}{3} \frac{V_{\text {rot }}^{2}}{R_{\star}}$,

which, of course, is independent of the inclination. Here and in the following we neglect any distortion of the stellar radius due to centrifugal forces that might become relevant in the case of rotational velocities close to break-up.

The geometrical situation is sketched in Fig. A.1. We consider two Cartesian co-ordinate systems, $\left(z_{*}, p_{*}, q_{*}\right)$ and $(z, p, q)$. The former refers to the stellar system with rotation axis $q_{*}$, and the latter to the one of the observer. The $z$-axis is directed towards the observer, the "impact parameter" $p=p_{*}$ is perpendicular to the $(z, q)$-plane (identical to the $\left(z_{*}, q_{*}\right)$-plane), and $q$ is the "height" of the disk, perpendicular to both $z$ and $p$. Thus, both planes are tilted with respect to one another in terms of inclination $i$ (between $q$ and $q_{*}$ ).

The co-ordinates in the stellar system can be expressed in terms of stellar co-latitude $\theta$, azimuthal angle $\phi$ and radius $r$. Any points, $\boldsymbol{r}$, on the stellar surface (and only those are needed for our calculation) can be described by the following relation:

$\boldsymbol{r}=\left(\begin{array}{c}z_{*} \\ p_{*} \\ q_{*}\end{array}\right)=\left(\begin{array}{c}R_{\star} \sin \theta \cos \phi \\ R_{\star} \sin \theta \sin \phi \\ R_{\star} \cos \theta\end{array}\right)$.
For the integration over the stellar disk, on the other hand, we first employ a polar co-ordinate system with co-ordinates $P$ and polar angle $\Phi$. Any point $\boldsymbol{d}$ situated on the stellar disk has then coordinates

$\boldsymbol{d}=\left(\begin{array}{l}p \\ q\end{array}\right)=\left(\begin{array}{c}P \cos \Phi \\ P \sin \Phi\end{array}\right), P=\sqrt{p^{2}+q^{2}}, \Phi=\arctan (q / p)$.

In this representation the desired average of the centrifugal acceleration over the stellar disk is given by

$\left\langle g_{\text {cent }}\right\rangle=\frac{\int_{0}^{2 \pi} \int_{0}^{R_{*}} g_{\text {cent }} P \mathrm{~d} P \mathrm{~d} \Phi}{\pi R_{\star}^{2}}$.

However, since $g_{\text {cent }}$ is dependent on the stellar co-latitude $\theta$, an integration with respect to the spherical stellar co-ordinates is advantageous.

Before we consider the general case of arbitrary inclination, we will deal with the simpler case where $\sin i=1$ (i.e., the star is observed equator-on). In this case, the stellar system (where $\theta$ is defined) and the system of the observer coincide, i.e., $q_{*}=q$, such that the transformation from $(P, \Phi)$ to $(\theta, \phi)$ is the following: Any projected point on the stellar disk can be represented by the corresponding physical location on the stellar sphere via

$\left(\begin{array}{l}p \\ q\end{array}\right)=\left(\begin{array}{c}P \cos \Phi \\ P \sin \Phi\end{array}\right)=\left(\begin{array}{c}R_{\star} \sin \theta \sin \phi \\ R_{\star} \cos \theta\end{array}\right)$,

$P^{2}=R_{\star}^{2}\left(\sin ^{2} \theta \sin ^{2} \phi+\cos ^{2} \theta\right)$

$\tan \Phi=\frac{\cot \theta}{\sin \phi}$

Note that we only have to consider the points on the half-sphere directed towards the observer, i.e., $z>0$. Since the integral over $P \mathrm{~d} P$ can alternatively be expressed by the integral over $1 / 2 \mathrm{~d} P^{2}$, the variable transformation inside the integrals, from $\left(\mathrm{d} P^{2}, \mathrm{~d} \Phi\right)$ to $(\mathrm{d} \theta, \mathrm{d} \phi)$, is obtained by evaluating the determinant of the Jacobian of the transformation (A.6) which (after a number of operations) turns out to be

$\operatorname{det}\left(J\left(\left(P^{2}, \Phi\right),(\theta, \phi)\right)\right)=2 R_{\star}^{2} \sin ^{2} \theta \cos \phi$.

Thus, for $\sin i=1$, the integral over the stellar disk can be expressed as an integral over the front of the stellar sphere,

$$
\begin{aligned}
\frac{1}{2} \int_{0}^{2 \pi} \int_{0}^{R_{*}^{2}} f\left(P^{2}, \Phi\right) \mathrm{d} P^{2} \mathrm{~d} \Phi= \\
R_{\star}^{2} \int_{-\pi / 2}^{\pi / 2} \int_{0}^{\pi} f(\theta, \phi) \sin ^{2} \theta \cos \phi \mathrm{d} \theta \mathrm{d} \phi .
\end{aligned}
$$

One can readily convince oneself that for $f(\theta, \phi)=1$ the correct result, $R_{\star}^{2} \pi$, is obtained, whereas for $f(\theta, \phi)=g_{\text {cent }}(\theta)$ we find

$\left\langle g_{\text {cent }}\right\rangle_{\text {disk, } \sin i=1}=\frac{3}{4} \frac{V_{\text {rot }}^{2}}{R_{\star}}$.

For arbitrary inclinations the line of argument is similar. Note that the relation between stellar and observer's system can be represented by a rotation with respect to the common $p$-axis, i.e.,

$\left(\begin{array}{l}z \\ p \\ q\end{array}\right)=\left(\begin{array}{ccc}\sin i & 0 & \cos i \\ 0 & 1 & 0 \\ -\cos i & 0 & \sin i\end{array}\right)\left(\begin{array}{c}z_{*} \\ p_{*} \\ q_{*}\end{array}\right)$ 
and the generalization of (A.6) is

$\left(\begin{array}{l}z \\ p \\ q\end{array}\right)=\left(\begin{array}{c} \\ P \cos \Phi \\ P \sin \Phi\end{array}\right)=\left(\begin{array}{c}R_{\star}(\sin \theta \cos \phi \sin i+\cos \theta \cos i) \\ R_{\star} \sin \theta \sin \phi \\ R_{\star}(-\sin \theta \cos \phi \cos i+\cos \theta \sin i)\end{array}\right)$,

$P^{2}=R_{\star}^{2}\left(\sin ^{2} \theta \sin ^{2} \phi+\sin ^{2} \theta \cos ^{2} \phi \cos ^{2} i+\cos ^{2} \theta \sin ^{2} i\right.$

$-2 \sin \theta \cos \theta \cos \phi \sin i \cos i)$

$\tan \Phi=\frac{-\cos \phi \cos i+\cot \theta \sin i}{\sin \phi}$

The corresponding determinant of the Jacobian becomes (calculated with MATHEMATICA)

$\operatorname{det}(J)=2 R_{\star}^{2} \sin \theta(\cos \theta \cos i+\sin \theta \cos \phi \sin i)$,

and for $\sin i=1$ we obtain the same result as above, whereas for $\sin i=0$ (i.e., the star is observed pole-on) we find (using appropriate integration limits, see below)

$$
\begin{aligned}
\left\langle g_{\text {cent }}\right\rangle_{\text {disk }, \sin \mathrm{i}=0} & =\frac{R_{\star}^{2} \int_{0}^{2 \pi} \int_{0}^{\pi / 2} g_{\text {cent }}(\theta) \sin \theta \cos \theta \mathrm{d} \theta \mathrm{d} \phi}{\pi R_{\star}^{2}} \\
& =\frac{1}{2} \frac{V_{\text {rot }}^{2}}{R_{\star}} .
\end{aligned}
$$

In order to obtain the complete expression for arbitrary $\sin i$, we first have to calculate the integration limits. From the condition $z>0$ (A.11) we find that

$i<\frac{\pi}{2}:\left\{\begin{array}{l}0<\phi<2 \pi, \\ 0<\theta<\theta_{\max }, \quad \theta_{\max }=\frac{-\cos \phi \tan i}{\sqrt{1+\cos ^{2} \phi \tan ^{2} i}}\end{array}\right.$

$i=\frac{\pi}{2}:\left\{\begin{aligned}-\pi / 2 & <\phi<\pi / 2, \\ 0 & <\theta<\pi\end{aligned}\right.$.

With these limits, we are able to calculate the first double inte$\operatorname{gral}(\propto \cos i, i<\pi / 2$, again with MATHEMATICA),

$\cos i \int_{0}^{2 \pi} \int_{0}^{\theta_{\max }} \sin ^{3} \theta \cos \theta \mathrm{d} \theta \mathrm{d} \phi=\frac{\pi}{8} \cos ^{2} i\left(4-2 \sin ^{2} i\right)$.

The second one ( $\propto \sin i, i<\pi / 2)$ is given by

$\sin i \int_{0}^{2 \pi} \int_{0}^{\theta_{\max }} \sin ^{4} \theta \cos \phi \mathrm{d} \theta \mathrm{d} \phi=\frac{\pi}{8} \sin ^{2} i\left(8-2 \sin ^{2} i\right) .($ A. 15$)$
Finally, we obtain the rather simple result

$\left\langle g_{\text {cent }}\right\rangle_{\text {disk }}(\sin i)=\frac{V_{\text {rot }}^{2}}{R_{\star}}\left(\frac{1}{2}+\frac{1}{4} \sin ^{2} i\right)$,

which includes the case $i=\pi / 2$ (and, of course, the pole-on case $i=0$ ).

In conclusion, the centrifugal acceleration averaged over the stellar disk depends on two terms. The first one, constituting a minimum value, depends on $V_{\text {rot }}^{2}$ alone (which cannot be measured) and a second term depends on $\left(V_{\mathrm{r}} \sin i\right)^{2}$ (which can be measured).

In order to obtain a suitable approximation for the centrifugal correction based on the measurable quantity $\left(V_{\mathrm{r}} \sin i\right)^{2}$, we will use appropriate means. Since the probability density function for the distribution of the inclination angle $i$ is given by $\sin i$ itself (see, e.g., Chandrasekhar \& Münch 1950), the mean and standard-deviation (i.e., square-root of the variance) of $\sin ^{2} i$ is given by

$\left\langle\sin ^{2} i\right\rangle=\frac{2}{3}\left(1 \pm \frac{1}{\sqrt{5}}\right)$.

By approximating $\left(V_{\mathrm{r}} \sin i\right)^{2}$ with $V_{\text {rot }}^{2}\left\langle\sin ^{2} i\right\rangle$, we can express the centrifugal correction via

$\left\langle g_{\text {cent }}\right\rangle \approx \frac{\left(V_{\mathrm{r}} \sin i\right)^{2}}{R_{\star}}\left(\frac{1}{2\left\langle\sin ^{2} i\right\rangle}+\frac{1}{4}\right)=\frac{\left(V_{\mathrm{r}} \sin i\right)^{2}}{R_{\star}}(1+\Delta f)$

$\Delta f=\frac{3}{4} \frac{1}{ \pm \sqrt{5}-1} \approx{ }_{-0.23}^{+0.61}$.

The larger error (i.e., an under-estimate of the centrifugal correction) occurs if $\sin ^{2} i<\left\langle\sin ^{2} i\right\rangle$ since $V_{\text {rot }}$ is large, whereas $V_{\mathrm{r}} \sin i$ is small. If $\sin ^{2} i \gtrsim\left\langle\sin ^{2} i\right\rangle$, the error is much lower because the estimator of $V_{\text {rot }}$ is of the correct order. Thus, we encounter the (somewhat paradoxical) result that our approximate centrifugal correction is rather correct for fast rotators (except for the still missing correction concerning the deformation of the stellar radius), whereas for "slow" rotators the centrifugal correction might be too low. 